\title{
Wilson loops and area laws in lattice gauge theory tensor networks
}

\author{
Erez Zohar (1) \\ Racah Institute of Physics, The Hebrew University of Jerusalem, Jerusalem 91904, Givat Ram, Israel
}

(Received 31 January 2021; accepted 3 August 2021; published 20 August 2021)

\begin{abstract}
Tensor network states have been a very prominent tool for the study of quantum many-body physics, thanks to their physically relevant entanglement properties and their ability to encode symmetries. In the last few years, the formalism has been extended and applied to theories with local symmetries too-lattice gauge theories. In order to extract physical properties (such as expectation values and correlation functions of physical observables) out of such states, one has to use the so-called transfer operators, the local properties of which dictate the long-range behavior of the state. In this paper we study transfer operators of tensor network states (in particular, projected entangled pair states) of lattice gauge theories, and consider the implications of the local symmetry on their structure and properties. In particular, we study the implications on the computation of the Wilson loop-a nonlocal, gauge-invariant observable which is central to pure gauge theories, the long-range decay behavior of which probes the confinement or deconfinement of static charges. Using the symmetry, we show how to simplify the tensor contraction required for computing Wilson loop expectation values for such states, eliminate nonphysical parts of the tensors, and formulate conditions relating local properties (that is, of the tensors) to their decay fashion.
\end{abstract}

DOI: 10.1103/PhysRevResearch.3.033179

\section{INTRODUCTION}

In recent years, tensor network states [1] have been a very prominent tool, rooted in quantum information science, for the study of quantum many-body systems and especially strongly correlated physics. In particular, matrix product states (MPSs) [2,3] enable one to study numerically and analytically physically relevant states, e.g., ground states of local many-body Hamiltonians (that is, states exhibiting an entanglement entropy area law). In higher spatial dimensions, MPSs generalize to projected entangled pair states (PEPSs) $[3,4]$. These are useful for the description of strongly correlated physics in two spatial dimensions and more.

PEPSs (and MPSs) are constructed out of the contraction of local building blocks (tensors). They satisfy, by construction, the entanglement entropy area law (focusing on the physically relevant part of the Hilbert space) and allow the state to depend on very few local parameters, hence making it feasible for computations (compared with arbitrary states in the exponentially large Hilbert space). Furthermore, they also allow one to encode symmetries already on the single tensor level. By properly parametrizing the local tensors, a global symmetry of the whole PEPS under a symmetry group can be imposed [5,6]. This way, one can generate families of ansatz states in which the symmetry group of the studied model is encoded by construction.

Published by the American Physical Society under the terms of the Creative Commons Attribution 4.0 International license. Further distribution of this work must maintain attribution to the author(s) and the published article's title, journal citation, and DOI.
While originally used mostly in the context of condensed matter physics, MPSs and PEPSs have recently been extended to the study of particle physics too-in particular, to lattice gauge theories, aiming at solving long standing open, nonperturbative questions of the standard model, such as the confinement of quarks [7]. Due to its running coupling [8], quantum chromodynamics (QCD), which allows one to use perturbation theory in high energy scales (collider physics) thanks to asymptotic freedom, is strongly interacting in low energies, preventing the use of perturbative methods. Lattice gauge theories (LGTs) [7] have been introduced to overcome this difficulty, first as tools for lattice regularization of gauge invariant field theories. They quickly became a very successful numerical approach. Combined with quantum Monte Carlo it has been applied to nonperturbative QCD computations, such as the hadronic spectrum [9]. However, quantum Monte Carlo does not allow for the direct observation of real time dynamics, and faces the fermionic sign problem in several important physical scenarios, not allowing one to probe some of the interesting exotic regions of the QCD phase diagram [10], and this requires the use of other methods, with tensor networks being one such approach. The tensor network framework for lattice gauge theories has been rapidly growing in the last few years.

For $1+1$-dimensional systems, MPSs have already been extensively used. This does not only include abstract formalistic descriptions of MPSs with a local symmetry [11,12] or benchmarks of models that can be treated in other ways, such as, but not only, the Schwinger model [13-20]. Successful numerical studies of lattice gauge theories in $1+1$ dimensions have been carried out even in scenarios which face the sign problem when approached with conventional methods (such as real time evolution [21-23] and finite density [24-27]). 
This was done for both Abelian and non-Abelian models-see [28] and references therein for a discussion of that.

The application of tensor networks to higher-dimensional lattice gauge theories has been discussed as well in the last few years [29-38]. From the rather more abstract, or formalistic, point of view, gauging mechanisms which lift globally invariant PEPSs to locally invariant ones by adding a gauge field and entangling it to the matter properly were introduced and discussed [39,40]. For a parallel approach in the action formalism - tensor field theory-which uses tensor networks (but not tensor network states), see [41] and references therein.

In this paper, we will focus on a particular gauging mechanism - the one introduced in [40] and used mostly with fermionic matter, for creating gauged Gaussian fermionic PEPSs [31,32]: special PEPS constructions which allow for the description of fermionic matter coupled to dynamical gauge fields. Their construction may be seen as a minimal coupling procedure on the level of states, which is not possible in general but could be done in the context of PEPSs [42]. While in general numerical computations are hard and challenging for PEPSs in two spatial dimensions and more, it has been shown that, when this particular construction is used, the PEPS may be contracted efficiently (allowing one to extract physical information) when combining with Monte Carlo methods which do not suffer from the sign problem [33]. Variational Monte Carlo then allows one to find ground states of lattice gauge theory Hamiltonians when such states are used as ansatz states, which has already been demonstrated and benchmarked for a pure $\mathbb{Z}_{3}$ lattice gauge theory in $2+1$ dimensions [37].

A question that has to be asked when a PEPS is studied is how physical information can be extracted from the contracted state-computation of expectation values of observables and correlation functions. Thanks to the special structure of MPSs, one may introduce a mathematical object called a transfer matrix (or operator) [43] to compute efficiently expectation values of observables and correlation functions. This may be extended to two-dimensional PEPSs, by first contracting the rows, obtaining effectively a chain of the rows which is an MPS, and considering its transfer matrix [44]. In this paper, we will study such transfer operators of lattice gauge theory PEPSs in two space dimensions.

Gauge theories are special in the sense that they exhibit a local symmetry, responsible to mediating local interactions between the matter fields. This symmetry gives rise to many local constraints. All the physical states are invariant under gauge transformations-local transformations parametrized by the elements of the so-called gauge group. As a result, only gauge invariant observables and correlation functionsthose which are invariant under local transformations-give rise to nonvanishing expectation values. Thus, LGT PEPSs admit a very special structure manifested in the local tensors $[30,39,40]$; in this paper, we focus on the implications of the local symmetry on the transfer operators, and hence aim at using the symmetry to simplify the PEPS contraction, focusing on pure gauge theories (that is, without dynamical matter).

In such scenarios, closed flux loops-usually referred to as the operators which create them, Wilson loops [7]-are perhaps the most important observables (and almost the only possible gauge invariant ones). The decay rule of large Wilson loops in pure gauge theories serves as probes for confinement of static charges: area law decay implies confinement, while a perimeter law implies a deconfined (Coulomb) phase. Confinement implies a gapped, disordered phase, while deconfined phases are massless and ordered [45]. The local ingredients of the Wilson loop are not gauge invariantonly their combination along the nonlocal path preserves the symmetry. This means that when computing it for a gauge invariant PEPS the transfer operator formalism must be extended and modified, requiring the inclusion of various types of transfer matrices which construct this nonlocal observable. The different building blocks will also have special properties [32], dictated by the special local symmetry, which will affect the behavior of the Wilson loop and its decay.

In this paper, we will study the properties of transfer operators of gauge invariant PEPS. We will see how the symmetry affects the properties of the local tensors, and that thanks to it some parts of the tensors may be excluded and ignored when a contraction is done (e.g., when combined with some numerical methods). We will also see how that affects the Wilson loop's decay-that is, how local properties of the tensors dictate the decay of large Wilson loops.

Note that PEPSs have been previously used for the computation of Wilson loop expectation value in various cases- $\mathbb{Z}_{2}$ string nets [46,47], as well as $U(1)$ [31] and $S U(2)$ [32] toy models; here we derive a general framework based on transfer matrix arguments and demonstrate with particular constructions.

We begin with briefly reviewing important preliminaries from group theory and lattice gauge theory, in Sec. II; we move on to formulating gauge invariant PEPSs and reviewing their symmetry properties, in Sec. III; in Sec. IV we introduce the transfer operators-after a brief review of their general properties, we formulate the flux-free transfer operators for LGT PEPSs, study their properties, and use them to calculate the norm; Sec. V focuses on the contraction of Wilson loop expectation values for LGT PEPSs, studying the relevant transfer operators and deriving conditions for area and perimeter decay laws; finally, in Sec. VI, we give an explicit illustration, including both analytical and numerical arguments, for a $\mathbb{Z}_{2}$ lattice gauge theory.

Throughout this paper the Einstein summation convention (on doubly repeated indices) is assumed unless stated otherwise; with the only exception of irreducible representation indices, the summation of which should not be assumed.

\section{MATHEMATICAL AND PHYSICAL PRELIMINARIES}

\section{A. Groups, transformations, and representations}

Consider a group $G$, which is either a finite or a compact Lie one. Each group element $g \in G$ may be represented by different unitary matrices $D^{j}(g)$, labeled by the group's irreducible representations (irreps) $j$; the dimension of these matrices is referred to as the irrep dimension, $\operatorname{dim}(j)$ [e.g., $2 j+1$ for $S U(2)]$.

In the Hilbert space $\mathcal{H}$ of some quantum mechanical theory, we would like to consider transformations parametrized by the elements of $G$. To do that, for each $g \in G$ we introduce 
a unitary operator $\theta_{g}$, and define it by its action on a basis state of the form $|j m\rangle$. $j$ labels the irreducible representations of $G$ and $m$ is an index labeling all states within this representation - that is, all the states that may be mixed by the transformations $\theta_{g}$ that act block diagonally on the irreps:

$$
\theta_{g}|j m\rangle=\left|j m^{\prime}\right\rangle D_{m^{\prime} m}^{j}(g) .
$$

We can hence express $\theta_{g}$ as

$$
\theta_{g}=\bigoplus_{j} D_{m n}^{j}(g)|j m\rangle\langle j n|
$$

and therefore the dimension of the irrep $j, \operatorname{dim}(j)$, is also the dimension of $\mathcal{H}_{j}$, the Hilbert subspace spanned by $|j m\rangle$, which we call a multiplet. The Hilbert space may be seen as a direct sum of multiplet subspaces:

$$
\mathcal{H}=\bigoplus_{j} \mathcal{H}_{j} .
$$

In general, quantum Hilbert spaces may contain more than one multiplet carrying the same irreducible representation.

These transformations are sometimes referred to as right transformations, since they mix the multiplet elements $|j m\rangle$, when seen as the components of a $\operatorname{dim}(j)$ dimensional vector, via right matrix multiplication, as shown in (1). One can also introduce the left transformations:

$$
\tilde{\theta}_{g}|j m\rangle=D_{m m^{\prime}}^{j}(g)\left|j m^{\prime}\right\rangle .
$$

Note that the left transformations are not independent from the right ones: for each $g \in G$ one may find $h$ such that $\tilde{\theta}_{g}=\theta_{h}$. We introduce the left transformations separately nevertheless since they will be mathematically convenient later when the PEPSs are constructed.

When $G$ is a compact Lie group, its elements may be uniquely identified in terms of group parameters or coordinates $\phi_{a}$; then, for each irrep $j$,

$$
D^{j}(g)=\exp \left[i \phi_{a}(g) T_{a}^{j}\right]
$$

the parameters $\phi_{a}(g)$ depend on the group element, while the generators $T_{a}^{j}$ depend on the representation. The latter form a set of matrices with dimension $\operatorname{dim}(j)$, satisfying the group's Lie algebra

$$
\left[T_{a}^{j}, T_{b}^{j}\right]=i f_{a b c} T_{c}^{j}
$$

where $f_{a b c}$ are the group's structure constants. One may also introduce the abstract generators, $J_{a}$, which are block diagonal in the representations,

$$
J_{a}=\bigoplus_{j}\left(T_{a}^{j}\right)_{m n}|j m\rangle\langle j n|,
$$

satisfying the algebra

$$
\left[J_{a}, J_{b}\right]=i f_{a b c} J_{c}
$$

too.

The states $|j m\rangle$ are eigenstates of mutually commuting operators: the $j$ quantum numbers(s) labeling the irreducible representation (and hence the multiplet) are eigenvalues of the Casimir operators which commute with all the generators; within the representation, the states are labeled by the eigenvalues of a maximal set of mutually commuting generators
(Cartan subalgebra) - $m$. Similarly, when the group is finite, $j$ labels the irreducible representation while the $m$ numbers are obtained from the simultaneous diagonalization of a maximal set of commuting transformations.

All the irreps of Abelian groups are one dimensional and thus no $m$ indices are required. In the $\mathbb{Z}_{N}$ case, the $N$ different irreps are labeled by the integers $j=0, \ldots, N-1$, which label the group elements $g=0, \ldots, N-1$ too, with $D^{j}(g)=$ $\exp \left(i \frac{2 \pi}{N} j g\right)$. In the $U(1)$ case the group elements are labeled by one parameter as well, $\phi \in[0,2 \pi)$; the representations are labeled by integers $j \in \mathbb{Z} ; D^{j}(\phi)=\exp (i j \phi)$; and $T^{j}=j \mathbf{1}$.

As a non-Abelian example, consider $S U(2)$, the irreps of which are labeled by $j$ that are non-negative integers and half integers. The dimension of each representation is $\operatorname{dim}(j)=$ $2 j+1$, and the $2 j+1$ within the multiplet are labeled by $m=-j, \ldots, j$. There are three generators, satisfying the Lie algebra with $f_{a b c}=\epsilon_{a b c}$ - the antisymmetric (Levi-Civita) symbol with $a, b, c=1,2,3$. The generators in this case are sometimes called the spin or angular momentum components, and then $a, b, c=x, y, z$. The $j=0$ (trivial) representation is one dimensional, with the singlet state $|00\rangle$. The next representation, $j=1 / 2$, is two dimensional ( $m= \pm 1 / 2)$, with generators proportional to the Pauli matrices, $T_{a}^{j=1 / 2}=\sigma_{a} / 2$. In this case, there is a single Casimir operator, $\mathbf{J}^{2}=J_{a} J_{a}$, commuting with one generator at most (the Cartan subalgebra is of size 1). Conventionally it is taken to be the $z$ or 3 component of the angular momentum, and thus for $S U(2)$

$$
\begin{aligned}
\mathbf{J}^{2}|j m\rangle & =j(j+1)|j m\rangle, \\
J_{z}|j m\rangle & =m|j m\rangle .
\end{aligned}
$$

\section{B. Lattice gauge theory basics}

Just like gauge theories in the continuum, LGTs describe the interaction of matter particles through gauge fields. In the lattice case, the matter fields reside on the lattice sites, while the gauge fields, mediating the interactions between matter particles, are on the links. One can either discretize both space and time [7], as used for Euclidean Monte Carlo computations, or discretize only space while keeping time continuous [48]. The latter corresponds to the Hamiltonian formulation widely used in the context of quantum simulation and tensor networks, including in this paper. Since we consider Hamiltonian lattice gauge theory in $2+1$ dimensions, our lattice will be two dimensional. As this paper focuses on the pure gauge case and matter fields are absent, all the degrees of freedom will reside on the links. We will review the basic ingredients of such models following the conventions of $[49,50]$.

\section{Local Hilbert spaces}

Consider a two-dimensional lattice, the sites of which are labeled by vectors of integers $\mathbf{x} \in \mathbb{Z}^{2}$. $\hat{\mathbf{e}}_{i}$ denote the unit vectors pointing in directions $i=1,2$, and any link is classified by two numbers, $(\mathbf{x}, i)$, standing for the beginning of the link and the direction to which it emanates, respectively. Each link $(\mathbf{x}, i)$ hosts a local gauge field Hilbert space $\mathcal{H}_{\text {gauge }}(\mathbf{x}, i)$, which can be spanned by group element states $\{|g\rangle\}_{g \in G}$ labeled by the gauge group elements. These states form a basis of 
$\mathcal{H}_{\text {gauge }}$, with the orthogonality relation

$$
\left\langle g^{\prime} \mid g\right\rangle=\delta\left(g^{\prime}, g\right),
$$

where $\delta\left(g^{\prime}, g\right)$ is the Kronecker delta if $G$ is finite, and a Dirac delta distribution in the compact lie case-denoting the Haar measure of $G$ by $d g$ :

$$
\int d g^{\prime} f\left(g^{\prime}\right) \delta\left(g^{\prime}, g\right)=f(g) .
$$

Unlike in the multiplet case, here the right and left transformations are independent of one another, as group multiplications: we introduce two sets of unitary operators, $\Theta_{g}$ and $\tilde{\Theta}_{g}$, parametrized by the elements of the gauge group $G$, which implement right and left group multiplications (respectively) on the group element states:

$$
\begin{aligned}
& \Theta_{g}|h\rangle=\left|h g^{-1}\right\rangle, \\
& \tilde{\Theta}_{g}|h\rangle=\left|g^{-1} h\right\rangle .
\end{aligned}
$$

The space $\mathcal{H}_{\text {gauge }}$ can also be spanned by the dual representation basis, the states of which are labeled by $|j m n\rangle-j$ is an irrep and $m, n$ are identifiers within it. In a sense, using the multiplet states introduced previously,

$$
|j m n\rangle=|j m\rangle \otimes|j n\rangle
$$

or

$$
\mathcal{H}_{\text {gauge }}=\bigoplus_{j} \mathcal{H}_{j} \otimes \mathcal{H}_{j}
$$

where $\mathcal{H}_{j}$ is the $\operatorname{dim}(j)$ dimensional subspace spanned by the $|j m\rangle$ multiplet states. We read this equation as a decomposition of the link's Hilbert space into a direct sum of products of multiplets of the groups on the left and right of the link, sharing the same irrep. Here one copy of each irreducible representation is used at most; one in the full, Kogut-Susskind case [48], but it is also possible to choose (for example, for reasons of feasibility of computation or experimental implementation) to truncate the sum and not include all the irreps in several ways $[49,51]$ as we will discuss later.

In the nontruncated case, using the Peter-Weyl theorem and the group's Fourier transform [49], the transition between the two bases is given by

$$
\langle g \mid j m n\rangle=\sqrt{\frac{\operatorname{dim}(j)}{|G|}} D_{m n}^{j}(g)
$$

where $|G|$ is the group's volume. In the representation basis,

$$
\begin{aligned}
\Theta_{g}|j m n\rangle & =\left|j m n^{\prime}\right\rangle D_{n^{\prime} n}^{j}(g), \\
\tilde{\Theta}_{g}|j m n\rangle & =D_{m m^{\prime}}^{j}(g)\left|j m^{\prime} n\right\rangle .
\end{aligned}
$$

In the compact Lie group case, one can introduce two sets of transformation generators, left and right $-L_{a}$ and $R_{a}$, respectively, such that

$$
\begin{aligned}
\Theta_{g} & =\exp \left[i \phi_{a}(g) R_{a}\right], \\
\tilde{\Theta}_{g} & =\exp \left[i \phi_{a}(g) L_{a}\right],
\end{aligned}
$$

satisfying the algebra

$$
\left[R_{a}, R_{b}\right]=i f_{a b c} R_{c}, \quad\left[L_{a}, L_{b}\right]=-i f_{a b c} L_{c}, \quad\left[R_{a}, L_{b}\right]=0 .
$$

Note that if the group is Abelian there is no difference between left and right operations and the indices $m, n$ do not exist. Therefore, there $R=L \equiv E$. Thus, in the $U(1)$ case, for example, we have group states labeled by the single compact parameter $|\phi\rangle$ and representation states labeled by the single integer $|j\rangle$, related through the Fourier series formula

$$
\langle\phi \mid j\rangle=\frac{1}{\sqrt{2 \pi}} e^{i j \phi}
$$

and the representation states $|j\rangle$ satisfy

$$
E|j\rangle=j|j\rangle \text {. }
$$

For $\mathbb{Z}_{N}$, similarly, we obtain the discrete Fourier series formula

$$
\langle g \mid j\rangle=\frac{1}{\sqrt{N}} e^{i 2 \pi j g / N} .
$$

In the $S U(2)$ case, since the group is non-Abelian, the situation is more complicated. There are $(2 j+1)^{2}|j m n\rangle$ states for each $j$-e.g., one singlet state $|000\rangle$ for $j=0$, and four $j=1 / 2$ states, $\left|\frac{1}{2}, \pm \frac{1}{2}, \pm \frac{1}{2}\right\rangle$. The group is parametrized by the three Euler angles $\alpha, \beta, \gamma$, and

$$
\langle\alpha, \beta, \gamma \mid j m n\rangle=\frac{\sqrt{2 j+1}}{8 \pi^{2}} D_{m n}^{j}(\alpha, \beta, \gamma) .
$$

The Hilbert space in this case is that of a rigid rotator $[48,52]$. The right and left operators $R_{a}$ and $L_{a}$ correspond to the generators of its rotations in the space and body frames of reference. These two sets of generators commute, and give rise to the same total angular momentum (eigenvalue of the Casimir operator) since it is a rotation scalar quantity which does not depend on the frame of reference [53,54]. Therefore,

$$
\begin{aligned}
\mathbf{J}^{2}|j m n\rangle \equiv \mathbf{R}^{2}|j m n\rangle & =\mathbf{L}^{2}|j m n\rangle=j(j+1)|j m n\rangle, \\
L_{z}|j m n\rangle & =m|j m n\rangle, \\
R_{z}|j m n\rangle & =n|j m n\rangle .
\end{aligned}
$$

\section{Local gauge invariance}

At each site $\mathbf{x}$, and for each group element $g \in G$, we introduce the gauge transformation

$$
\hat{\Theta}_{g}(\mathbf{x})=\tilde{\Theta}_{g}(\mathbf{x}, 1) \tilde{\Theta}_{g}(\mathbf{x}, 2) \Theta_{g}^{\dagger}\left(\mathbf{x}-\hat{\mathbf{e}}_{1}, 1\right) \Theta_{g}^{\dagger}\left(\mathbf{x}-\hat{\mathbf{e}}_{1}, 2\right)
$$

which transforms all the four links intersecting at $\mathbf{x}$ with respect to the same group element - the outgoing links with the left transformation, and the ingoing ones with the inverse right one. The outgoing links, the beginning (left) side of which connects to $\mathbf{x}$, undergo a left rotation, while the ingoing ones, connected through their end (right) side to $\mathbf{x}$, undergo an inverse right rotation.

A gauge invariant state $|\psi\rangle$ satisfies

$$
\hat{\Theta}_{g}(\mathbf{x})|\psi\rangle=|\psi\rangle, \quad \forall \mathbf{x} \in \mathbb{Z}^{2}, g \in G
$$

(see Fig. 1) and similarly, for a gauge invariant operator $O$,

$$
\hat{\Theta}_{g}(\mathbf{x}) O \hat{\Theta}_{g}^{\dagger}(\mathbf{x})=O, \quad \forall \mathbf{x} \in \mathbb{Z}^{2}, g \in G
$$



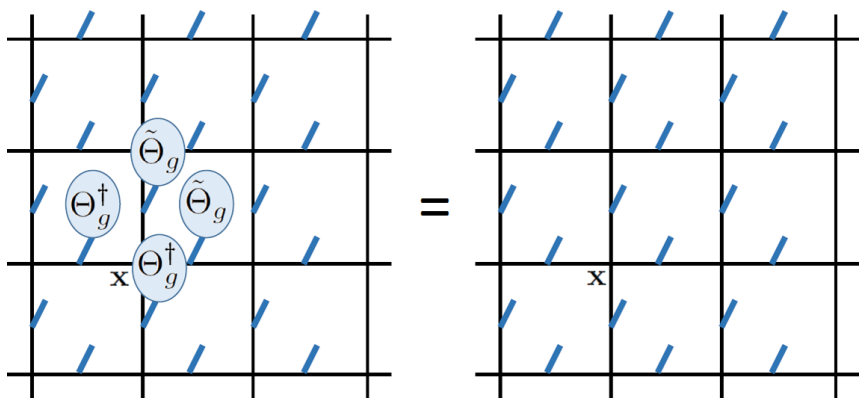

FIG. 1. Gauge transformations act on the four links around a vertex with a particular set of unitary transformations parametrized by the same group element $g \in G$.

(one can extend it to the case of static charges [52] which we do not discuss here). In a lattice gauge theory, only gauge invariant states and operators are considered physical.

If $G$ is a compact Lie group, we can formulate the gauge transformations $\hat{\Theta}_{g}(\mathbf{x})$ in terms of their generators

$$
\begin{aligned}
\mathcal{G}_{a}(\mathbf{x})= & L_{a}(\mathbf{x}, 1)+L_{a}(\mathbf{x}, 2)-R_{a}\left(\mathbf{x}-\hat{\mathbf{e}}_{1}, 1\right) \\
& -R_{a}\left(\mathbf{x}-\hat{\mathbf{e}}_{2}, 2\right) .
\end{aligned}
$$

Gauge invariance is then formulated in terms of the Gauss laws

$$
\mathcal{G}_{a}(\mathbf{x})|\psi\rangle=0, \quad \forall \mathbf{x} \in \mathbb{Z}^{2}, a
$$

(once again, excluding static charges [52]).

We call this eigenvalue equation the Gauss law, since $\mathcal{G}_{a}(\mathbf{x})$ can clearly be seen as the divergence of electric fields $-L_{a}$ and $R_{a}$ - on a site. For physical states-the ones which satisfy the local constraints (25) - the divergence of electric fields is zero. It is very apparent in the $U(1)$ case, where it takes the explicit form

$$
\begin{aligned}
& \left(E(\mathbf{x}, 1)+E(\mathbf{x}, 2)-E\left(\mathbf{x}-\hat{\mathbf{e}}_{1}, 1\right)-E\left(\mathbf{x}-\hat{\mathbf{e}}_{2}, 2\right)\right)|\psi\rangle \\
& \quad \equiv \nabla \cdot \mathbf{E}(\mathbf{x})|\psi\rangle=0, \quad \forall \mathbf{x} \in \mathbb{Z}^{2}, a .
\end{aligned}
$$

In non-Abelian cases, the divergence involves left and right electric fields, which is related to the charge carried by nonAbelian gauge bosons [48] (e.g., the color charged gluon vs the electric neutral photon).

\section{Wilson loops}

Since we deal with gauge invariant states, it is expected that the expectation values of non-gauge-invariant operators will vanish. Thus, when classifying the phases and behavior of gauge theories one needs to consider only gauge invariant observables and correlation functions.

One option, for the compact Lie group, is to compute expectation values of electric field operators and functions thereof (and only of Casimir operators if the group is nonAbelian). Another possible gauge invariant observable is the loop variable, and in particular Wilson loops [7].

On the local link Hilbert spaces we introduce the group element operators:

$$
U_{m n}^{j}=\int d g D_{m n}^{j}(g)|g\rangle\langle g| .
$$

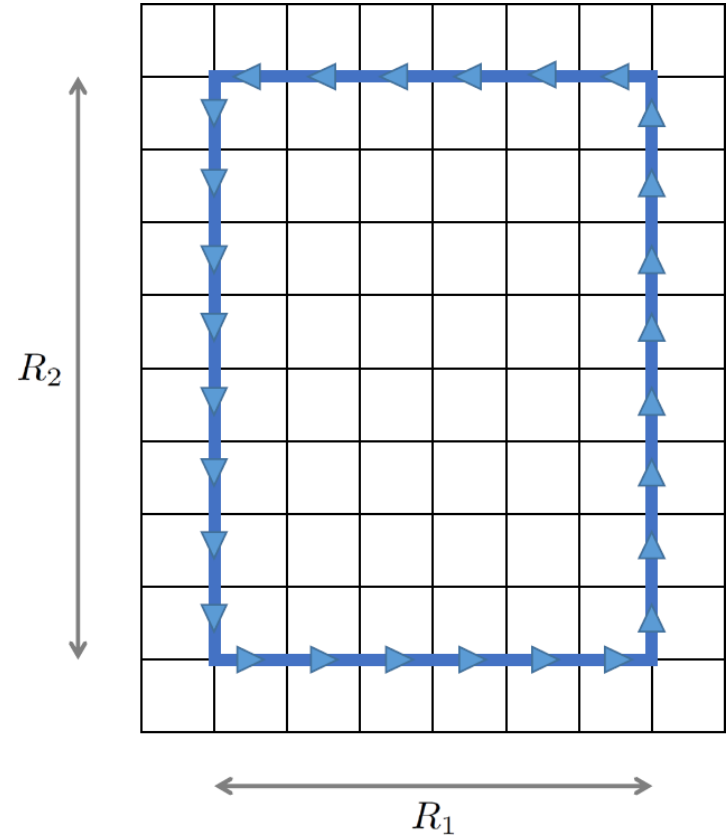

FIG. 2. A Wilson loop: rectangular loop of electric flux. On links in the positive directions (pointing rightwards and upwards, here on the lower and right edges of the loop) the group element operator $U$ is used; on links in the negative directions (pointing leftwards and downwards, here on the left and upper edges of the loop), $U^{\dagger}$ is used.

$U^{j}$ is a matrix of $\operatorname{dimension} \operatorname{dim}(j) \times \operatorname{dim}(j)$, the elements of which are operators acting on the link's gauge field Hilbert space, $\mathcal{H}_{\text {gauge }}$ [on each link $\ell$ we can define such operators $\left.U_{m n}^{j}(\ell)\right]$. Even though they are Hilbert space operators, all the elements of $U^{j}$ commute - one can see in the definition above that they are all diagonal in the same basis. The matrix elements of $U^{j}$ mix with respect to the transformation properties of the $j$ representation,

$$
\begin{aligned}
& \Theta_{g} U_{m n}^{j} \Theta_{g}^{\dagger}=U_{m n^{\prime}}^{j} D_{n^{\prime} n}^{j}(g), \\
& \tilde{\Theta}_{g} U_{m n}^{j} \tilde{\Theta}_{g}^{\dagger}=D_{m m^{\prime}}^{j}(g) U_{m^{\prime} n}^{j},
\end{aligned}
$$

and, in the compact Lie case,

$$
\begin{aligned}
& {\left[R_{a}, U_{m n}^{j}\right]=U_{m n^{\prime}}^{j}\left(T_{a}^{j}\right)_{n^{\prime} n},} \\
& {\left[L_{a}, U_{m n}^{j}\right]=\left(T_{a}^{j}\right)_{m m^{\prime}} U_{m^{\prime} n}^{j} .}
\end{aligned}
$$

Let us take some closed path $\mathcal{C}$ on the lattice. We define the Wilson loop operator $\mathcal{W}(\mathcal{C})$ as the ordered contraction of group element operators along this closed path, that is,

$$
\begin{aligned}
\mathcal{W}^{j}(\mathcal{C}) & =\operatorname{Tr}\left(\prod_{\ell \in \mathcal{C}} U^{j}(\ell)\right) \\
& =U^{j}\left(\ell_{1}\right)_{m_{1} m_{2}} U^{j}\left(\ell_{2}\right)_{m_{2} m_{3}} \cdots U^{j}\left(\ell_{L}\right)_{m_{L} m_{1}} .
\end{aligned}
$$

It is a trace over the product of the group element operators $U$, seen as matrices, ordered along the closed path $\mathcal{C}$ with length $L$ (which is simply the number of links along the path). Depending on the orientation of the path, one may have to use $U^{\dagger}$ instead of $U$, on half of the links along the path-those pointing leftwards or downwards (see Fig. 2). For simplicity, 
we will omit the $j$ indices below, but obviously the same irrep must be used along the path, otherwise the matrix product is ill defined. Consider $U(1)$ with $j=1$ as an example; there,

$$
\mathcal{W}(\mathcal{C})=\exp \left(i \sum_{\ell \in \mathcal{C}} \phi(\ell)\right)
$$

with half of the phases with a minus sign, according to their orientation.

In order to consider the action of the group element operators on representation states, we use the Clebsch-Gordan series and coefficients $\langle J M j m \mid K N\rangle$ [55] and obtain

$$
\begin{aligned}
& U_{m m^{\prime}}^{j}\left|J M M^{\prime}\right\rangle \\
& \quad=\sqrt{\frac{\operatorname{dim}(J)}{\operatorname{dim}(K)}}\langle J M j m \mid K N\rangle\left\langle K N^{\prime} \mid J M^{\prime} j m^{\prime}\right\rangle\left|K N N^{\prime}\right\rangle ;
\end{aligned}
$$

that is, the action of the group element operator $U^{j}$ on a state with representation $J$ yields states with all representations which are obtained by combining $j$ and $J$ (more precisely, fusing the two irreps together). Acting with a loop operator hence excites the representations along the loop with respect to that rule. One may truncate the Hilbert space in the representation basis: as long as all the irreducible representations used are taken completely and connected by nonzero Clebsch Gordan coefficients when $j$ is added, one may use (35) to define a $U^{j}$ operator acting on that truncated space. The transformation properties (16), (31), and (32) will still hold [49], which may make it convenient for some numerical approaches (or quantum simulation implementations [56]) but, since the group structure will be lost, the group element basis will no longer be defined, making, in particular, (30) and the Fourier transform (15) invalid.

In most cases, rectangular Wilson loops are considered. We denote by $W\left(R_{1}, R_{2}\right)$ a rectangular loop sized $R_{1} \times R_{2}$ (see Fig. 2). Very large Wilson loops of pure gauge theories are a probe for confinement (or deconfinement) of static charges, as introduced by Wilson in [7] (see also [45,57,58]). In a confining phase,

$$
-\log \left\langle W\left(R_{1}, R_{2}\right)\right\rangle \propto R_{1} R_{2}
$$

for $R_{1}, R_{2} \gg 1$ (area law), while in a deconfined phase

$$
-\log \left\langle W\left(R_{1}, R_{2}\right)\right\rangle \propto R_{1}+R_{2}
$$

for $R_{1}, R_{2} \gg 1$ (perimeter law).

In [59], Creutz introduced the parameter

$$
\chi\left(R_{1}, R_{2}\right)=-\log \left(\frac{W\left(R_{1}, R_{2}\right) W\left(R_{1}-1, R_{2}-1\right)}{W\left(R_{1}-1, R_{2}\right) W\left(R_{1}, R_{2}-1\right)}\right)
$$

for the detection of static charge confinement. In the general case of

$$
\left\langle W\left(R_{1}, R_{2}\right)\right\rangle=W_{0} e^{-\kappa_{A} R_{1} R_{2}-\kappa_{P}\left(R_{1}+R_{2}\right)},
$$

for large $R_{1}, R_{2}$, the area factor $\kappa_{A}$ (called the string tension), should it exist, is the most dominant one. The Creutz parameter $\chi$ filters out the contributions of the constant prefactor $W_{0}$ and the perimeter coefficient $\kappa_{P}$, and thus within a confining phase, $\chi\left(R_{1}, R_{2}\right) \rightarrow \kappa_{A}>0$ for $R_{1}, R_{2} \gg 1$, while in a deconfining one it converges to zero.

\section{GAUGE INVARIANT PEPSs}

In this paper, we use the lattice gauge theory PEPS formalism of $[33,40]$, with slightly different notations (and restricted to the pure gauge case). First of all, let us review it.

\section{A. Review of the PEPS construction}

Each site $\mathbf{x} \in \mathbb{Z}^{2}$ of our square, periodic lattice is at the intersection of four legs. The outgoing ones are in the right and up directions, while the left and down directed legs are considered ingoing. We wish, as usual with PEPSs, to construct a physical lattice state describing different physical degrees of freedom located on different sites. Each such degree of freedom is described by a local physical Hilbert space: if we had matter, we would fix a physical matter Hilbert space to each lattice site. Here, however, the gauge fields are our only physical degrees of freedom, and they reside on the links. Thus, with each lattice site $\mathbf{x}$ we associate two physical Hilbert spaces, located on the outgoing legs. We refer to them as the side $\left(\mathcal{H}_{s}\right)$ and top $\left(\mathcal{H}_{t}\right)$ physical Hilbert spaces.

These are local gauge field Hilbert spaces (note that the word local here has to do with being defined on a single link, not with the gauge symmetry being local) - that is, either the full $\mathcal{H}_{\text {gauge }}$ spaces introduced in Eq. (14), or truncated versions thereof containing only some representations. When truncating, it is important to make sure that all the $|j m n\rangle$ for an included $j$ are present, otherwise no gauge invariance can be imposed, as explained above [40,49].

When constructing a PEPS, in order to connect the local physical building blocks to one physical quantum state, one has to introduce auxiliary or virtual degrees of freedom, on top of the physical ones given by the model we study. These are used merely for the purpose of contraction. On each of the four legs we introduce an auxiliary or virtual Hilbert space, $\mathcal{H}_{r}, \mathcal{H}_{u}, \mathcal{H}_{l}, \mathcal{H}_{d}$ for the right, up, left, and down going legs, respectively. They are spanned by group multiplet states of the form $|j m\rangle$, as defined in Eq. (3). One may include all such multiplets, truncate, or include several copies of the same multiplet, which allows one to increase the number of variational parameters; but once again, all the states within a multiplet included must be present, and the representations used in the physical spaces must be included (though possibly with a higher multiplicity). For more details about that, refer to [40] where the general construction of such states is discussed.

On each site, we construct the physical-virtual state

$$
\begin{aligned}
|A\rangle= & A_{j_{r} m_{r} ; j_{u} m_{u} ; j_{l} m_{l} ; j_{d} m_{d}}^{j_{s} m_{n} n_{s} ; j_{m} m_{m} n_{t}} \\
& \times\left|j_{s} m_{s} n_{s} ; j_{t} m_{t} n_{t}\right\rangle\left|j_{r} m_{r} ; j_{u} m_{u} ; j_{l} m_{l} ; j_{d} m_{d}\right\rangle \\
\in & \mathcal{H}_{s} \times \mathcal{H}_{t} \times \mathcal{H}_{r} \times \mathcal{H}_{u} \times \mathcal{H}_{l} \times \mathcal{H}_{d}
\end{aligned}
$$

where the first ket refers to the physical states and the second refers to the virtual ones (see Fig. 3). The coordinate $\mathbf{x}$ was omitted for simplicity, but the Hilbert spaces are all associated with particular sites and, in general, the tensors $A_{j_{r} m_{r} ; j_{u} m_{u} ; j_{l} m_{l} ; j_{d} m_{d}}^{j_{s} m_{3} ; j_{t} m_{t} n_{t}}$ may depend on the position, although we will focus on translationally invariant PEPSs and thus they will be independent of $\mathbf{x}$. 

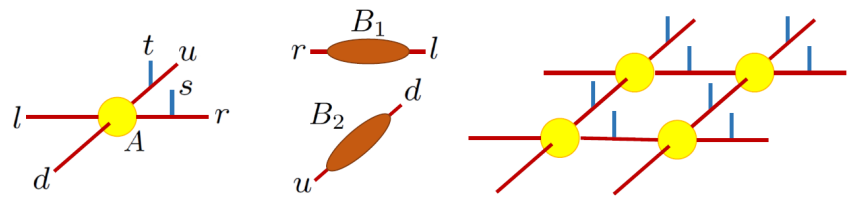

FIG. 3. The building blocks of the PEPS: on the left, the site tensors $A$ (40), with the physical legs $s, t$ and the virtual ones $r, u, l, d$. In the middle, the link projectors, $B_{1,2}(41)$, connecting the outgoing legs $r, u$ with the ingoing legs $l, d$ of the next sites, to the right and above, respectively. On the right, the contracted PEPS $|\psi\rangle$ (42), obtained after the projection.

To contract the PEPS, on each link we introduce the maximally entangled states

$$
\begin{aligned}
& \left|B_{1}(\mathbf{x})\right\rangle=\sum_{j}|j m\rangle_{r, \mathbf{x}}|j m\rangle_{l, \mathbf{x}+\hat{\mathbf{e}}_{1}}, \\
& \left|B_{2}(\mathbf{x})\right\rangle=\sum_{j}|j m\rangle_{u, \mathbf{x}}|j m\rangle_{d, \mathbf{x}+\hat{\mathbf{e}}_{2}} .
\end{aligned}
$$

As usual, we construct our PEPS $|\psi\rangle$ by projecting the virtual states on the legs onto the maximally entangled states:

$$
|\psi\rangle=\bigotimes_{\mathbf{x}, i}\left\langle B_{i}(\mathbf{x})\left|\bigotimes_{\mathbf{x}}\right| A(\mathbf{x})\right\rangle .
$$

Note that $\bigotimes_{\mathbf{x}}|A(\mathbf{x})\rangle$ in both the physical and virtual spaces, while $\underset{\mathbf{x}, i}{\bigotimes}\left\langle B_{i}(\mathbf{x})\right|$ is only virtual. Thus the result of this projection, $|\psi\rangle$, is still a quantum state, including only physical degrees of freedom - the virtual, or auxiliary ones, are all contracted: this is the standard way to contract PEPSs, and a frequently used notation. The physical degrees of freedom are now correlated, and in particular, thanks to maximally entangling nearest neighbors, this guarantees the entanglement entropy area law.

One still has some freedom to choose which maximally entangled states to use; the ones that we picked here are invariant under the following group transformations:

$$
\begin{aligned}
\tilde{\theta}_{g}^{r}(\mathbf{x}) \theta_{g}^{l^{\dagger}}\left(\mathbf{x}+\hat{\mathbf{e}}_{1}\right)\left|B_{1}(\mathbf{x})\right\rangle=\left|B_{1}(\mathbf{x})\right\rangle, \\
\tilde{\theta}_{g}^{u}(\mathbf{x}) \theta_{g}^{d^{\dagger}}\left(\mathbf{x}+\hat{\mathbf{e}}_{2}\right)\left|B_{2}(\mathbf{x})\right\rangle=\left|B_{2}(\mathbf{x})\right\rangle
\end{aligned}
$$

[with $\theta_{g}, \tilde{\theta}_{g}$ defined in (1) and (4), respectively] as depicted in Fig. 4. This allows one to construct states with a global or local symmetry, as we shall now see.

\section{B. Imposing the local symmetry}

We want our PEPS $|\psi\rangle$ (42) to be gauge invariant as in (25) with respect to the local gauge transformations defined in (24). If the local physical-virtual states on each site
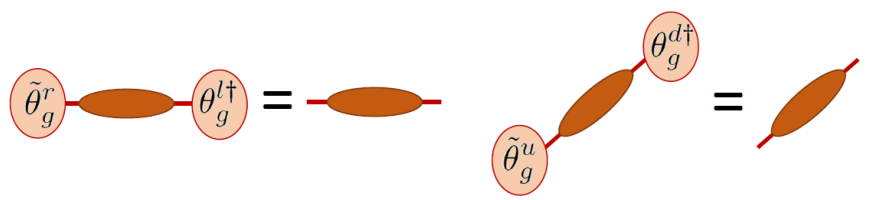

FIG. 4. The invariance properties of the links. satisfy [40]

$$
\begin{aligned}
& \tilde{\Theta}_{g}^{s}(\mathbf{x}) \tilde{\Theta}_{g}^{t}(\mathbf{x})|A(\mathbf{x})\rangle=\theta_{g}^{l}(\mathbf{x}) \theta_{g}^{d}(\mathbf{x})|A(\mathbf{x})\rangle, \\
& \Theta_{g}^{s}(\mathbf{x})|A(\mathbf{x})\rangle=\tilde{\theta}_{g}^{r}(\mathbf{x})|A(\mathbf{x})\rangle, \\
& \Theta_{g}^{t}(\mathbf{x})|A(\mathbf{x})\rangle=\tilde{\theta}_{g}^{u}(\mathbf{x})|A(\mathbf{x})\rangle, \quad \forall g \in G,
\end{aligned}
$$

where the physical Hilbert spaces are transformed using $\Theta, \tilde{\Theta}$ defined in (16), and the virtual ones using $\Theta, \tilde{\Theta}$ defined in (1) and (4), respectively (see Fig. 5), then using the transformation properties of the maximally entangled states (43) one obtains that $|\psi\rangle$ is gauge invariant.

In order to get a more intuitive picture of the symmetry conditions (44), let us consider the compact Lie group case again. Omitting the coordinate, since we deal with a single coordinate $\mathbf{x}$, let us denote the right and left generators of the physical degrees of freedom by $R_{a}^{s / t}$ and $L_{a}^{s / t}$. For the virtual degrees of freedom we can also define such operators, but in their case note that they do not commute, since they do not act on separate degrees of freedom $(|j m\rangle$ states, unlike the physical $|j m n\rangle$ states). The conditions (44) can be expressed, using these notations, as Gauss laws:

$$
\begin{aligned}
\left(L_{a}^{s}+L_{a}^{t}\right)|A\rangle & =\left(R_{a}^{l}+R_{a}^{d}\right)|A\rangle, \\
R_{a}^{s}|A\rangle & =L_{a}^{r}|A\rangle, \\
R_{a}^{t}|A\rangle & =L_{a}^{u}|A\rangle \quad \forall a .
\end{aligned}
$$

The first condition looks like the familiar physical Gauss law. It implies that the two ingoing representations of the virtual indices must combine to the same representation to which the two physical representations combine: $j_{s} \otimes j_{t} \sim$ $j_{l} \otimes j_{d}$. Therefore, the tensor $A_{j_{r} m_{r} ; j_{u} m_{u} ; j_{l} m_{l} ; j_{d} m_{d}}^{j_{s} m_{n} n_{j_{1}} m_{l} n_{t}}$ should be proportional to the appropriate Clebsch-Gordan coefficients, $\left\langle j_{l} m_{l} j_{d} m_{d} \mid j_{1} m_{1}\right\rangle\left\langle j_{1} m_{1} \mid j_{s} m_{s} j_{t} m_{t}\right\rangle$.

The other two conditions are different, identifying the right constituents of the physical degrees of freedom with the virtual states on the same legs. This implies that $j_{r}=j_{s}, j_{u}=j_{t}$, $m_{r}=n_{s}$, and $m_{u}=n_{t} ; A_{j_{r} m_{r} ; j_{u} m_{u} ; j_{l} m_{l} ; j_{d} m_{d}}^{j_{s} m_{s} n_{s} ; j_{t} m_{t} n_{t}}$ must be proportional to $\delta_{j_{s} j_{r}} \delta_{j_{t} j_{t}} \delta_{n_{s}, m_{r}} \delta_{n_{t}, m_{u}}$. Combining the first condition with the other two, we can obtain a condition on the four virtual legs: the elements of $A_{j_{r} m_{r} ; j_{u} m_{u} ; j_{l} m_{l} ; j_{d} m_{d}}^{j_{s} m_{3} n_{s} ; m_{t} m_{1} n_{1}}$ must vanish, unless

$$
j_{r} \otimes j_{u} \sim j_{l} \otimes j_{d} .
$$

Examples for constructions satisfying that have been previously given $[31,32,40,42]$; let us just briefly comment on some special cases. When the group is Abelian, only the irrep indices remain and the Clebsch-Gordan coefficients are simply Kronecker deltas. One can then formulate $j_{r} \otimes j_{u} \sim j_{l} \otimes j_{d}$ in a very simple way. For $U(1)$, for example, $\left\langle j_{1} j_{2} \mid J\right\rangle=$ $\delta_{j_{1}+j_{2}, J}$, and the $\mathbb{Z}_{N}$ is the appropriate modular modification, $\left\langle j_{1} j_{2} \mid J\right\rangle=\delta_{j_{1}+j_{2}, J \bmod N}$. We thus obtain, in the $U(1)$ case, only tensor elements for which $j_{r}+j_{u}-j_{l}-j_{d}=0$ may be nonzero $\left(j_{r}+j_{u}-j_{l}-j_{d}=N \mathbb{Z}\right.$ for $\left.\mathbb{Z}_{N}\right)$.

The same applies to non-Abelian groups as well, but since physical states contain the (generally different) $m, n$ quantum numbers it is less simple. For $S U(2)$, e.g., if we choose to include only the $j=0,1 / 2$ representations, the only nonvanishing tensor elements will be those with an even number of virtual legs (ingoing or outgoing) with $j=1 / 2$, such that a 


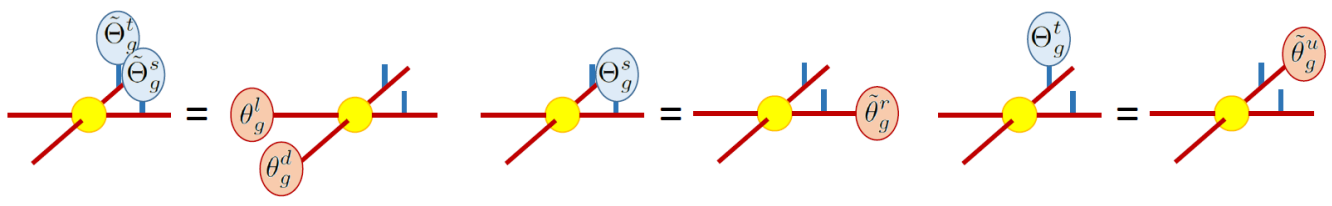

FIG. 5. The invariance properties of the tensors (44), allowing for a physical local (gauge) symmetry.

singlet can be formed by combining the contributions of all four legs.

The only freedom left in the definition of $A_{j_{r} m_{r} ; j_{u} m_{u} ; j_{l} m_{l} ; j_{d} m_{d}}^{j_{s} m_{s} n_{s} ; j_{t} m_{n_{1}} n_{t}}$ is to introduce some parameters $f_{j_{r}, j_{u}, j_{l}, j_{d}}^{j}$ which only depend on the representations, and we obtain [40]

$$
\begin{aligned}
A_{j_{r} m_{r} ; j_{u} m_{u} ; j_{l} m_{l} ; j_{d} m_{d}}^{j_{s} m_{s} n_{i} ; j_{j} m_{t} n_{t}}= & \sum_{j} f_{j_{r}, j_{u}, j_{l}, j_{d}}^{j}\left\langle j_{l} m_{l} j_{d} m_{d} \mid j m\right\rangle \\
& \times\left\langle j m \mid j_{s} m_{s} j_{t} m_{t}\right\rangle \delta_{j_{s} j_{r}} \delta_{j_{t} j_{t}} \delta_{n_{s}, m_{r}} \delta_{n_{t}, m_{u}} .
\end{aligned}
$$

In the following, we will focus on PEPSs satisfying the above symmetry properties, with no more than one copy of each irrep in the virtual spaces. This may seem restrictive when attempting to apply the states to real, physically relevant Hamiltonians; here, however, we wish to consider the most minimal constructions which capture the relevant symmetry properties, allowing us to demonstrate our claims and results as accurately as possible. When applied to Hamiltonians as variational ansatz states the states may have to be generalized indeed but in a straightforward way that does not affect the properties we discuss here. For example, as was demonstrated already in the $\mathbb{Z}_{3}$ case [37], several copies of the virtual representations are required in order to use such PEPSs in order to variationally find the ground states of the $\mathbb{Z}_{3}$ Hamiltonian.

One could also consider a more general PEPS construction, in which such properties are only satisfied after blocking, for effective sites and effective links. The symmetry conditions described above will hold in this case too-for the blocked tensor network, rather than the original, "microscopic" one, and thus what we study here could easily be applied to such cases too. A more general scenario would be with local matrix product operator symmetries [4], but this is out of the scope of this paper and requires its own, separate discussion.

\section{Tensor notation}

The projection (42) which generates the PEPS $|\psi\rangle$ can simply be seen as a set of contraction rules for the virtual indices of the tensors $A_{j_{r} m_{r} ; j_{u} m_{u} ; j_{l} m_{l} ; j_{d} m_{d}}^{j_{s} m_{1} n_{s} ; j_{t} m_{m_{1}} n_{1}}$, associating the indices of $r$ at $\mathbf{x}$ with those of $l$ at $\mathbf{x}+\hat{\mathbf{e}}_{1}$, as well as $u$ at $\mathbf{x}$ with $d$ at $\mathbf{x}+\hat{\mathbf{e}}_{2}$. Hence instead of looking at the local states $|A\rangle$ and their projection onto the link states $\left|B_{i}(x)\right\rangle$, we may use, as our basic local building block,

$$
A=A_{\text {ruld }}^{s_{1} s_{2} ; t_{1} t_{2}}|l\rangle\langle r|\otimes| d\rangle\left\langle u|| s_{1} s_{2} t_{1} t_{2}\right\rangle
$$

where, for the sake of notation simplicity, $r \equiv$ $\left\{j_{r}, m_{r}\right\}, u \equiv\left\{j_{u}, m_{u}\right\}, l \equiv\left\{j_{l}, m_{l}\right\}, d \equiv\left\{j_{d}, m_{d}\right\}, s_{1} \equiv$ $\left\{j_{s}, m_{s}\right\}, s_{2} \equiv\left\{j_{s}, n_{s}\right\}, \quad t_{1} \equiv\left\{j_{t}, m_{t}\right\}, t_{2} \equiv\left\{j_{t}, n_{t}\right\}, \quad$ and $A_{\text {ruld }}^{s_{1} s_{2} ; t_{1} t_{2}} \equiv A_{j_{r} m_{r} ; j_{u} m_{u} ; j_{l} m_{l} ; j_{d} m_{d}}^{j_{s} m_{s} n_{s} ; j_{t} m_{t} n_{t}}$. In (48) the virtual states and their projection are replaced by the matrix products of $|l\rangle\langle r|$ along horizontal lines (with the positive direction from the left to the right) and $|d\rangle\langle u|$ on the vertical lines (positive direction-upwards). This sets the contraction rules of the tensors $A_{\text {ruld }}^{s_{1} s_{2} ; t_{1} t_{2}}$.

To illustrate, let us reduce to one space dimension and onedimensional PEPS - an MPS [43]. Each local tensor along the one-dimensional system includes one physical leg, spanned by states $|p\rangle$, and two virtual ones, on the left and right direction. The state is thus parametrized by the tensors $A_{l r}^{p}$, and their contraction is simply a matrix multiplication of the virtual indices along the system. For a periodic system with $\mathcal{N}$ sites (the modification for open boundaries is straightforward) the state takes the form

$$
\begin{aligned}
& \left|\psi_{0}\right\rangle=\operatorname{Tr}\left[A^{p_{1}} A^{p_{2}} \cdots A^{p_{\mathcal{N}}}\right]\left|p_{1}, p_{2}, \ldots, p_{\mathcal{N}}\right\rangle
\end{aligned}
$$

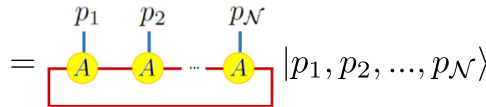

The PEPS contraction rules in two space dimensions are simply a two-dimensional generalization of the trace contraction in the one-dimensional case.

The symmetry conditions (44) may also be expressed as properties of the tensor $A_{j_{r} m_{r} ; j_{u} m_{u} ; j_{l} m_{l} ; j_{d} m_{d}}^{j_{s} m_{s} n_{s} ; j_{t} m_{2} m_{t}}$. For that, we introduce the (reducible) representation matrices $\mathcal{D}(g)$ which are direct sums of the irreducible unitaries $D^{j}(g)$; using them, the symmetry condition (44) may be reformulated as

$$
\begin{aligned}
A_{\text {ruld }}^{s_{1}^{\prime} s_{2} ; t_{1}^{\prime} t_{2}} \mathcal{D}_{s_{1}^{\prime} s_{1}}(g) \mathcal{D}_{t_{1}^{\prime} t_{1}}(g)=\mathcal{D}_{l l^{\prime}}(g) \mathcal{D}_{d d^{\prime}}(g) A_{r u l^{\prime} d^{\prime}}^{s_{1} s_{2} ; t_{1} t_{2}}, \\
\mathcal{D}_{s_{2} s_{2}^{\prime}}(g) A_{\text {ruld }}^{s_{1} s^{\prime} ; t_{1} t_{2}}=A_{r^{\prime} u l d}^{s_{1} s_{2} ; t_{1} t_{2}} \mathcal{D}_{r^{\prime} r}(g), \\
\mathcal{D}_{t_{2} t_{2}^{\prime}}(g) A_{\text {ruld }}^{s_{1} s_{2} ; t_{1}^{\prime} t_{2}}=A_{r u^{\prime} l d}^{s_{1} s_{2} ; t_{1} t_{2}} \mathcal{D}_{u^{\prime} u}(g) \quad \forall g \in G .
\end{aligned}
$$

\section{TRANSFER OPERATORS AND NORMS OF PEPS}

Before turning to the study of the transfer operator of our gauge invariant PEPS, let us recall what the transfer operator of a PEPS is. First, we briefly review the one-dimensional MPS case [43]. We strictly focus on the translationally invariant case, since this paper is aimed at translational invariant systems; however, the general transfer matrix discussion may be (and has been) generalized to the nontranslationally invariant case.

\section{A. Brief review of MPS transfer matrices}

The transfer matrix of the MPS $\left|\psi_{0}\right\rangle$ from (49) is defined as

$$
E_{l l^{\prime}, r r^{\prime}}=\operatorname{Tr}_{\text {phys }}\left[A_{l r}^{p}|p\rangle\left\langle p^{\prime}\right| \bar{A}_{l^{\prime} r^{\prime}}^{p^{\prime}}\right]=A_{l r}^{p} \bar{A}_{l^{\prime} r^{\prime}}^{p}=\left.\right|_{l-A-r} ^{l^{\prime}-\bar{A}-r^{\prime}}
$$


it is a matrix with double valued indices, $l l^{\prime}$ to the left and $r r^{\prime}$ to the right. Thus, if $l, r$ take $D$ values each (i.e., the virtual Hilbert spaces used for contracting the MPS are $D$ dimensional), $E$ is a $D^{2} \times D^{2}$ matrix, acting on the $D^{2}$ space formed by the product of two copies of the virtual Hilbert space. Using $E$, we can first write down the norm of the state:

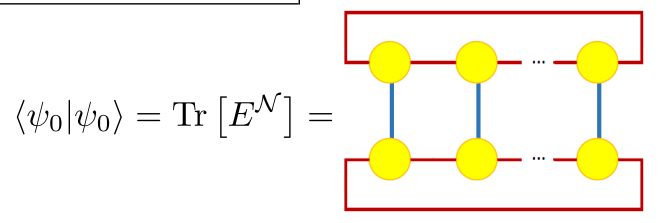

For the computation of an expectation value of some operator $O$ at site $x$, we will need to define

$$
\left(E_{O}\right)_{l l^{\prime}, r r^{\prime}}=\operatorname{Tr}_{\text {phys }}\left[A_{l r}^{p} O|p\rangle\left\langle p^{\prime}\right| \bar{A}_{l^{\prime} r^{\prime}}^{p^{\prime}}\right]=\sum_{p, p^{\prime}} A_{l r}^{p} \bar{A}_{l^{\prime} r^{\prime}}^{p^{\prime}}\left\langle p^{\prime}|O| p\right\rangle={\stackrel{\bar{O}}{l^{\prime}-\bar{A}-r^{\prime}}}_{l-A-r}^{\widehat{A}-r}
$$

using which we may write

$$
\langle O(x)\rangle=\frac{\left\langle\psi_{0}|O(x)| \psi_{0}\right\rangle}{\left\langle\psi_{0} \mid \psi_{0}\right\rangle}=\frac{\operatorname{Tr}\left[E_{O} E^{\mathcal{N}-1}\right]}{\operatorname{Tr}\left[E^{\mathcal{N}}\right]}=\frac{\ldots}{2}
$$

Suppose we wish to compute the two-point correlator of $O_{1}\left(x_{1}\right)$ and $O_{2}\left(x_{2}\right)$ (assuming for simplicity that $x_{2}-x_{1}=R>0$ ),

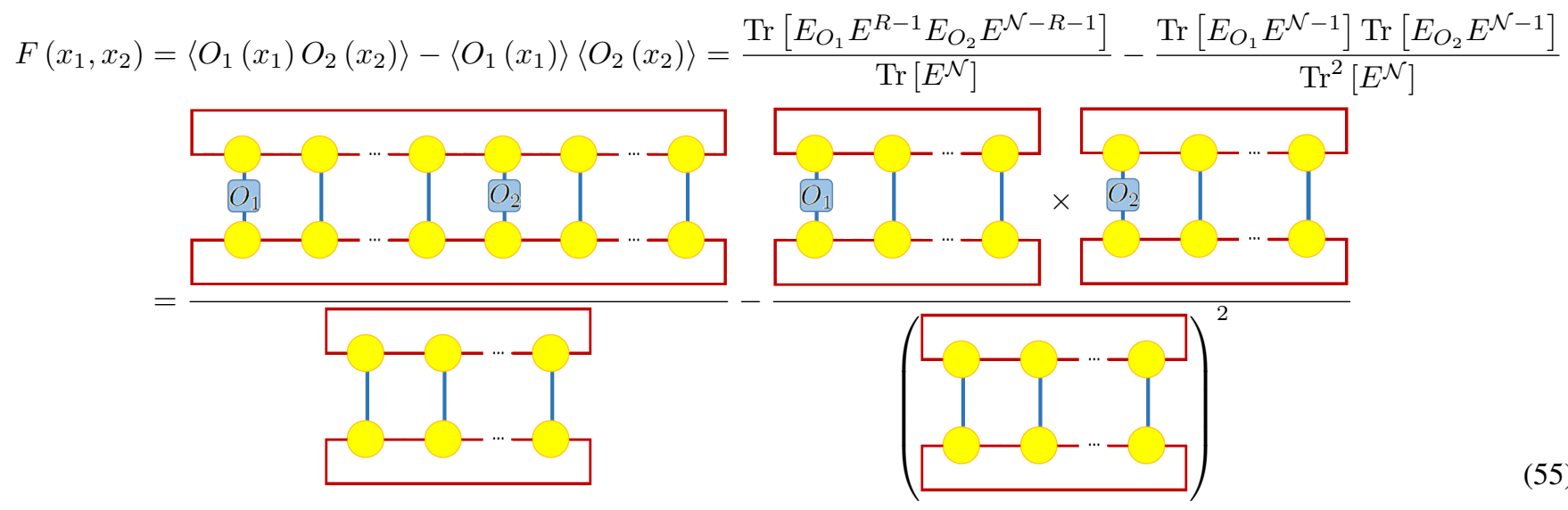

We introduce the left and right eigenvectors of $E,\left\langle w_{i}\right| E=$ $\left\langle w_{i}\right| \rho_{i}$ and $E\left|v_{i}\right\rangle=\rho_{i}\left|v_{i}\right\rangle$, sharing the same eigenvalues $\rho_{i}$ and satisfying the orthonormality relation $\left\langle w_{i} \mid v_{j}\right\rangle=\delta_{i j}$, and expand $E$ as

$$
E=\sum_{i} \rho_{i}\left|v_{i}\right\rangle\left\langle w_{i}\right| .
$$

Let us sort the eigenvalues in a descending order and assume that the largest one is nondegenerate, that is, $\left|\rho_{1}\right|>\left|\rho_{2}\right| \geqslant$ $\left|\rho_{3}\right| \geqslant \ldots$ Then, for $N \gg 1, R \gg 1$, one obtains that

$$
F\left(x_{1}, x_{2}\right) \approx \rho_{1}^{-2} \sum_{i>1}\left(\frac{\rho_{i}}{\rho_{1}}\right)^{R-1}\left\langle w_{1}\left|E_{O_{1}}\right| v_{i}\right\rangle\left\langle w_{i}\left|E_{O_{2}}\right| v_{1}\right\rangle
$$

the correlations decay exponentially, with a finite correlation length $\xi=-1 / \log \left|\frac{\rho_{2}}{\rho_{1}}\right|$.

\section{B. Transfer operators of PEPSs}

The transfer matrix approach can be generalized to twodimensional PEPSs, such as the ones we consider here, constructed in (42). We assume the system has periodic boundary conditions-a torus of size $\mathcal{N} \times \mathcal{N}$ (generalizations to other boundary conditions are straightforward). The local transfer operator of a PEPS on a site is a map from two double virtual Hilbert spaces, associated with the ingoing (left and down) legs, to two other double virtual spaces, directed to the 
outgoing directions (right and up):

$$
\hat{T}=T_{l l^{\prime}, r r^{\prime}, d d^{\prime}, u u^{\prime}}\left|l l^{\prime}\right\rangle\left\langle r r^{\prime}|\otimes| d d^{\prime}\right\rangle\left\langle u u^{\prime}\right|
$$

(note that we use again a convention in which the input vectors are denoted by bras, in accordance with the matrix product ordered from left to right in the positive system directions).

In full analogy with the one-dimensional case, the elements of the transfer tensor $T_{l l^{\prime}, r r^{\prime}, d d^{\prime}, u u^{\prime}}$ are given by

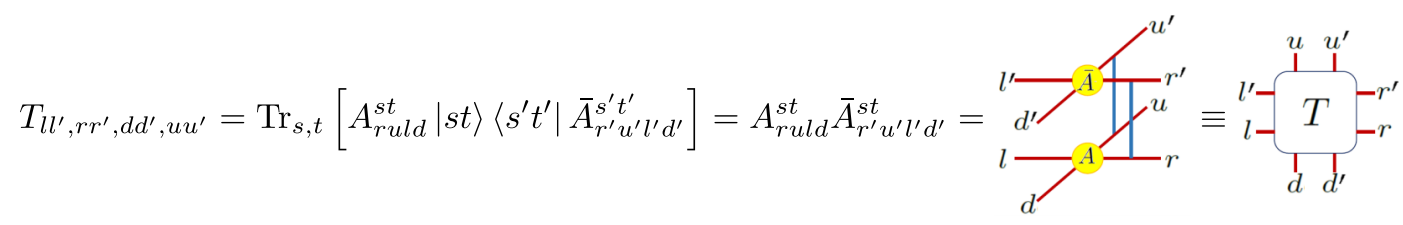

The norm may be computed by properly contracting products of $\hat{T}$ on all the lattice sites; in expectation values of observables, the numerator may be computed by replacing $\hat{T}$ at the relevant sites by

$$
\left(T_{O}\right)_{l l^{\prime}, r r^{\prime}, d d^{\prime}, u u^{\prime}}=\operatorname{Tr}_{\text {phys }}\left[A_{r u l d}^{s t} O|s t\rangle\left\langle s^{\prime} t^{\prime}\right| \bar{A}_{r^{\prime} u^{\prime} l^{\prime} d^{\prime}}^{s^{\prime} t^{\prime}}\right]=A_{r u l d}^{s t} \bar{A}_{r^{\prime} u^{\prime} l^{\prime} d^{\prime}}^{s^{\prime} t^{\prime}}\left\langle s^{\prime} t^{\prime}|O| s t\right\rangle=\underbrace{}_{l}-\underbrace{T_{O}}_{d^{\prime}} s_{-r}^{r^{\prime}}
$$

To compute correlations, we will first contract all the tensors along one dimension of the PEPS, converting it effectively to an MPS $[31,32,44]$ the transfer matrix of which can be defined as above. For example, the transfer matrix of a row of length $\mathcal{N}$ is obtained by contraction along the horizontal direction,

$$
\begin{aligned}
& \hat{E}=T_{l_{1} l_{1}^{\prime}, r_{1} r_{1}^{\prime}, d_{1} d_{1}^{\prime}, u_{1} u_{1}^{\prime}} T_{l_{2} l_{2}^{\prime}, r_{2} r_{2}^{\prime}, d_{2} d_{2}^{\prime}, u_{2} u_{2}^{\prime}} \cdots T_{l_{\mathcal{N}} l_{\mathcal{N}}^{\prime}, r_{\mathcal{N}} r_{\mathcal{N}}^{\prime}, d_{\mathcal{N}} d_{\mathcal{N}}^{\prime}, u_{\mathcal{N}} u_{\mathcal{N}}^{\prime}} \operatorname{Tr}\left[\left|l_{1} l_{1}^{\prime}\right\rangle\left\langle r_{1} r_{1}^{\prime} \mid l_{2} l_{2}^{\prime}\right\rangle\left\langle r_{2} r_{2}^{\prime}|\cdots| l_{\mathcal{N}} l_{\mathcal{N}}^{\prime}\right\rangle\left\langle r_{\mathcal{N}} r_{\mathcal{N}}^{\prime}\right|\right] \\
& \times\left|d_{1} d_{1}^{\prime}\right\rangle\left\langle u_{1} u_{1}^{\prime}|\otimes \ldots \otimes| d_{\mathcal{N}} d_{\mathcal{N}}^{\prime}\right\rangle\left\langle u_{\mathcal{N}} u_{\mathcal{N}}^{\prime}\left|=E_{d_{1} d_{1}, \ldots, d_{\mathcal{N}}, d_{\mathcal{N}}^{\prime} ; u_{1} u_{1}^{\prime}, \ldots, u_{\mathcal{N}}, u_{\mathcal{N}}^{\prime}}\right| d_{1} d_{1}^{\prime}\right\rangle\left\langle u_{1} u_{1}^{\prime}|\otimes \ldots \otimes| d_{\mathcal{N}} d_{\mathcal{N}}^{\prime}\right\rangle\left\langle u_{\mathcal{N}} u_{\mathcal{N}}^{\prime}\right|
\end{aligned}
$$

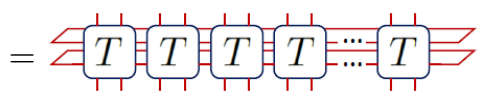

where

$$
\begin{aligned}
& E_{d_{1} d_{1}, \ldots, d_{\mathcal{N}}, d_{\mathcal{N}}^{\prime} ; u_{1} u_{1}^{\prime}, \ldots, u_{\mathcal{N}}, u_{\mathcal{N}}^{\prime}} \\
& \quad=T_{i_{1}, i_{1}^{\prime}, i_{2} i_{2}^{\prime}, d_{1} d_{1}^{\prime}, u_{1} u_{1}^{\prime}} T_{i_{2}, i_{2}^{\prime}, i_{3} i_{3}^{\prime}, d_{2} d_{2}^{\prime}, u_{2} u_{2}^{\prime}} \cdots T_{i_{\mathcal{N}} i_{\mathcal{N}}^{\prime}, i_{1} i_{1}^{\prime}, d_{\mathcal{N}} d_{\mathcal{N}}^{\prime}, u_{\mathcal{N}} u_{\mathcal{N}}^{\prime} .} .
\end{aligned}
$$

Using $E$ and similar transfer matrices which include observables, one may use the entire MPS machinery for computations of norms, expectation values, and correlation functions. Naively, one may deduce that correlations in this case decay exponentially as in the MPS case [43]. However, unlike in the one-dimensional MPS case, here the transfer matrix is a composite object with some internal structure, which can lead to different results. It was shown in [60], for example, that two-dimensional PEPSs can describe critical physics, exhibiting power law contributions.

\section{Flux-free transfer operators}

Let us apply the above to the computation of the norm. For that, consider the flux-free transfer operator, that is, the local building block of the transfer matrix on a single site, with no string (group element operator $U^{j}$ ), $\hat{T}$, as defined in (58). We calculate its elements using (59), and thanks to the symmetry conditions (50) we obtain that for every $g \in G$

$$
\left(\theta_{g}^{l} \otimes \tilde{\theta}_{g}^{\dagger l^{\prime}}\right) \otimes\left(\theta_{g}^{d} \otimes \tilde{\theta}_{g}^{\dagger d^{\prime}}\right) \hat{T}=\hat{T} \theta_{g}^{r} \otimes \tilde{\theta}_{g}^{\dagger r^{\prime}}=\hat{T} \theta_{g}^{u} \otimes \tilde{\theta}_{g}^{\dagger u^{\prime}}=\hat{T}
$$

[see Fig. 6(a)]. This implies that in (58) the outgoing vectors $\left|r r^{\prime}\right\rangle$ and $\left|u u^{\prime}\right\rangle$ are both separately singlets under the action of $\left(\theta_{g} \otimes \tilde{\theta}_{g}^{\dagger}\right)$ - that is, they are on-leg singlets, denoted by $\left\langle 0\left(j_{r}\right)\right|$ and $\left\langle 0\left(j_{u}\right)\right|$ and defined as

$$
|0(j)\rangle=|j m j m\rangle .
$$

The ingoing legs $\left|l l^{\prime}\right\rangle \otimes\left|d d^{\prime}\right\rangle$, on the other hand, combine together to a singlet under $\left(\theta_{g}^{l} \otimes \tilde{\theta}_{g}^{\dagger l^{\prime}}\right) \otimes\left(\theta_{g}^{d} \otimes \tilde{\theta}_{g}^{\dagger d^{\prime}}\right)$ : $\left\langle j_{l} m_{l} j_{d} m_{d} \mid j_{l}^{\prime} m_{l}^{\prime} j_{d}^{\prime} m_{d}^{\prime}\right\rangle\left|j_{l} m_{l}, j_{l}^{\prime} m_{l}^{\prime}\right\rangle \otimes\left|j_{d} m_{d}, j_{d}^{\prime} m_{d}^{\prime}\right\rangle$. We can therefore conclude that the general structure of $\hat{T}$ is

$$
\begin{aligned}
\hat{T}= & \sum_{\{j\}} T_{j_{l}, j_{l}^{\prime}, j_{r} ; j_{d}, j_{d}^{\prime}, j_{u}}\left\langle j_{l} m_{l} j_{d} m_{d} \mid j_{l}^{\prime} m_{l}^{\prime} j_{d}^{\prime} m_{d}^{\prime}\right\rangle\left|j_{l} m_{l}, j_{l}^{\prime} m_{l}^{\prime}\right\rangle\left\langle 0\left(j_{r}\right)\right| \\
& \otimes\left|j_{d} m_{d}, j_{d}^{\prime} m_{d}^{\prime}\right\rangle\left\langle 0\left(j_{u}\right)\right| ;
\end{aligned}
$$

it is a map with two inputs and two outputs, which takes a joint singlet (on both the ingoing legs) into two separate on-leg singlets, on each outgoing leg alone [see Fig. 6(b)].

$$
\theta_{g} \otimes \tilde{\theta}_{g}^{\dagger}-\underbrace{T+1}_{\theta_{g} \otimes \tilde{\theta}_{g}^{\dagger}}=\theta_{g}^{+1} \otimes \tilde{\theta}_{g}^{\dagger}=
$$

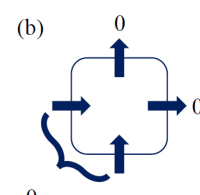

FIG. 6. The invariance properties of the transfer operator $\hat{T}$ (a) and its map interpretation (b). 


\section{Row transfer matrix and the norm}

Suppose we wish to compute the norm, which involves contracting the tensor product of $\hat{T}$ everywhere. Each $\hat{T}$ obtains its inputs from the neighboring $\hat{T}$ operators on its left and bottom, the outputs of which are on-leg singlets: that is, when the norm is computed, the inputs $\left|j_{l} m_{l}, j_{l}^{\prime} m_{l}^{\prime}\right\rangle\left\langle 0\left(j_{r}\right)\right|$ on the left leg and $\left|j_{d} m_{d}, j_{d}^{\prime} m_{d}^{\prime}\right\rangle\left\langle 0\left(j_{r}\right)\right|$ on the lower one are being contracted with the outputs from neighboring sites- $\left\langle 0\left(j_{r}\right)\right|$ and $\left\langle 0\left(j_{u}\right)\right|$, respectively. Thus, for the norm contraction it is enough to focus only on a subset of the $T$ elements, where only on-leg singlets are allowed as input. Denoting by $\Pi_{0}=$ $\sum_{j}|0(j)\rangle\langle 0(j)|$ the projection operator onto on-leg singlets $|0(j)\rangle=|j m j m\rangle$, we define

$$
\hat{\tau}_{0}=\Pi_{0} \otimes \Pi_{0} \hat{T} \equiv
$$

(introducing a new notation which will be used for tiling diagrams below, in which the legs are implicit). It takes the simple form

$$
\hat{\tau}_{0}=\sum_{\{j\}}\left(\tau_{0}\right)_{j_{l}, j_{r} ; j_{d}, j_{u}}\left|0\left(j_{l}\right)\right\rangle\left\langle 0\left(j_{r}\right)|\otimes| 0\left(j_{d}\right)\right\rangle\left\langle 0\left(j_{u}\right)\right|
$$

where $\left(\tau_{0}\right)_{j_{l}, j_{r} ; j_{d}, j_{u}}=T_{j_{l}, j_{l}, j_{r} ; j_{d}, j_{d}, j_{u}}$.

To see how this simplifies the contraction, let us consider some illustrative examples. First, consider the $\mathbb{Z}_{N}$ case, in which (disregarding multiplicities) the virtual Hilbert spaces are spanned by $D=N$ basis states, corresponding to the $j=$ $0, \ldots, N-1$ irreps. Thus we will have $N$ on-leg singlets, of the form

$$
|0(j)\rangle=|j j\rangle .
$$

The tensor $\hat{\tau}_{0}$ will thus contain $N^{4}$ elements; having considered $T$ without taking the symmetry into account, with two $N$-dimensional legs per direction, we would have instead $N^{8}$ tensor elements. That is, the number of elements that actually need to be used for contraction is $N^{4}$ times smaller. Next, generalize to $U(1)$, and suppose we truncate and allow for the $|j| \leqslant J$ for some $J>0$. Then we will have once again on-leg singlets of the form (68). There are $D=2 J+1$ irreps in the virtual Hilbert space, we have $D$ on-leg singlets, and, similarly to the $\mathbb{Z}_{N}$ case, we obtain a reduction of $D^{4}: D^{4}$ elements in $\hat{\tau}_{0}$ which we need for the contraction, rather than the $D^{8}$ in the most general case.

The simplification is even bigger when we consider non-Abelian groups, because the tensors $\hat{\tau}_{0}$ only see the representations and not the different $m$ values within them. For example, consider $S U(2)$, with the smallest truncation, containing the $j=0,1 / 2$ representations. This implies that each virtual Hilbert space has dimension 3. Naively speaking, $T$ would be a tensor with $3^{8}=6561$ elements. Reducing to $\hat{\tau}_{0}$, with only two on-leg singlets for the two irreps used, the number of relevant elements decreases to $2^{4}=16$, that is, approximately 410 times less. If we wish to consider, a little more generally, all the irreps of $S U$ (2) between zero and some $J$, the dimension of the virtual Hilbert spaces would be $D(J)=\sum_{j=0}^{J}(2 j+1)=(J+1)(2 J+1)$ (note that the sum runs on both integer and half-integer values). Thus $T$ has $D^{8}(J)=(J+1)^{8}(2 J+1)^{8}$ elements. However the number of on-leg singlets is as the number of irreps, $2 J+1$, and hence $\hat{\tau}_{0}$ is a tensor with $(2 J+1)^{4}$ elements: the reduction factor is $(J+1)^{8}(2 J+1)^{4}$, which scales as $J^{-12}$ for large cutoffs-a very significant reduction.

To examine further the properties of $\hat{\tau}_{0}$, let us consider $\left(\tau_{0}\right)_{j_{l}, j_{r} ; j_{d}, j_{u}}$ as a matrix with the multivalued indices $j_{l}, j_{r}$ and $j_{u}, j_{d}$. If we assume horizontal-vertical reflection symmetry, we find that it is a symmetric matrix:

$$
\left(\tau_{0}\right)_{j_{l}, j_{r} ; j_{d}, j_{u}}=\left(\tau_{0}\right)_{j_{d}, j_{u} ; j_{l}, j_{r}}
$$

Furthermore, it is a real matrix, since using (59), with the restriction (67), we obtain that

$$
\begin{aligned}
& \left(\tau_{0}\right)_{j_{l}, j_{r} ; j_{d}, j_{u}}=T_{j_{l}, j_{l}, j_{r} ; j_{d}, j_{d}, j_{u}}
\end{aligned}
$$

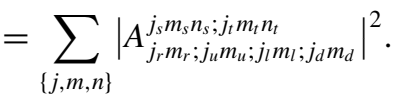

Therefore, there exists an orthogonal matrix $V$, such that

$$
\tau_{0}=V \Lambda V^{\dagger}
$$

where $\Lambda$ is a diagonal matrix with eigenvalues $\lambda_{\mu}$. This allows us to bring $\hat{\tau}_{0}$ to the convenient form

$$
\hat{\tau}_{0}=\sum_{\mu} \lambda_{\mu} \hat{M}_{\mu} \otimes \hat{M}_{\mu}
$$

where

$$
\hat{M}_{\mu}=\sum_{j_{1}, j_{2}} V_{j_{1} j_{2}, \mu}\left|0\left(j_{1}\right)\right\rangle\left\langle 0\left(j_{2}\right)\right|,
$$

one copy of which acts on the horizontal direction and the other copy of which acts on the virtual one.

The real matrices $\left\{\hat{M}_{\mu}\right\}$ form an orthonormal set with respect to the trace inner product. Since $V$ is orthogonal, it is straightforward to show that

$$
\operatorname{Tr}\left[\hat{M}_{\mu} \hat{M}_{\nu}^{T}\right]=\delta_{\mu \nu} .
$$

Supposing our tensor includes $D$ irreps, all the $j$ s take $D$ different values. Then there are $D$ different on-leg singlets, and the matrix $\tau_{0}$ is $D^{2} \times D^{2}$; thus, $\mu=1, \ldots, D^{2}$ and we have $D^{2} \hat{M}_{\mu}$ matrices. They act on the $D$-dimensional space spanned by the $D$ linearly independent on-leg singlets $|0(j)\rangle$. These matrices form a $D^{2}$ linear space; we have shown that $\hat{M}_{\mu}$ is an orthonormal set of $D^{2}$ matrices within this space, and thus it is an orthonormal basis and the $\hat{M}_{\mu}$ span the whole space of $D \times D$ real matrices.

The row transfer matrix and the norm thus take the forms

$$
\begin{aligned}
\hat{E} & \equiv \quad=\operatorname{Tr}_{\text {row }}\left[\begin{array}{lllll} 
& & \cdots & \\
1 & 2 & & \\
&
\end{array}\right. \\
& =\sum_{\{\mu\}} \lambda_{\mu_{1}} \lambda_{\mu_{2}} \cdots \lambda_{\mu_{\mathcal{N}}} \operatorname{Tr}\left[\hat{M}_{\mu_{1}} \hat{M}_{\mu_{2}} \cdots \hat{M}_{\mu_{\mathcal{N}}}\right] \hat{M}_{\mu_{1}} \otimes \hat{M}_{\mu_{2}} \otimes \cdots \otimes \hat{M}_{\mu_{\mathcal{N}}}
\end{aligned}
$$


and

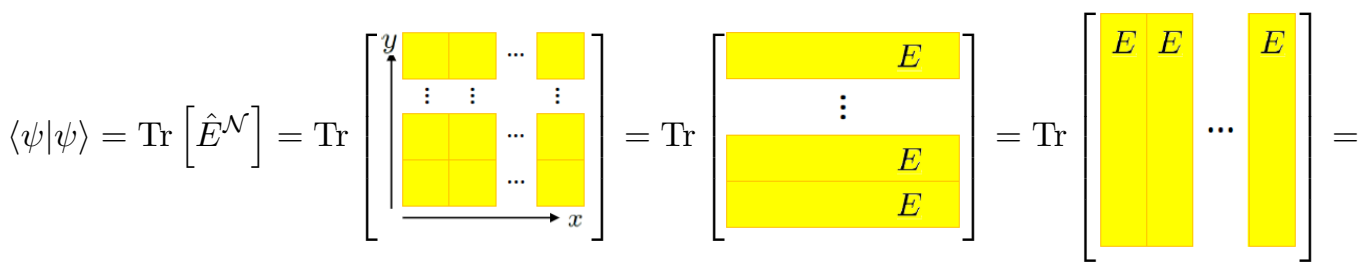

$$
\begin{aligned}
& =\sum_{\{\mu(x, y)\}} \prod_{x, y} \lambda_{\mu(x, y)} \prod_{y} \operatorname{Tr}\left[\hat{M}_{\mu(1, y)} \hat{M}_{\mu(2, y)} \cdots \hat{M}_{\mu(\mathcal{N}, y)}\right] \prod_{x} \operatorname{Tr}\left[\hat{M}_{\mu(x, 1)} \hat{M}_{\mu(x, 2)} \cdots \hat{M}_{\mu(x, \mathcal{N})}\right]
\end{aligned}
$$

\section{E. Spectrum of the flux-free transfer matrix}

We have used the fact that each leg of $\hat{\tau}_{0}$ forms a singlet $|0(j)\rangle$; however, recall the symmetry properties of the tensor $A$ out of which the transfer operators were constructed, and the Gauss law satisfied by its four legs (46): $j_{r} \otimes j_{u} \sim j_{l} \otimes j_{d}$. This implies that $\left(j_{l} \otimes j_{r}\right) \otimes\left(j_{d} \otimes j_{u}\right)$ must contain the single representation: the horizontal representations and the vertical ones must be such that can fuse to a singlet together. As a consequence of that, elements of $\left(\tau_{0}\right)_{j_{l}, j_{r} ; j_{d}, j_{u}}$ the indices of which do not satisfy it must vanish. This splits the matrix $\left(\hat{\tau}_{0}\right)_{j_{l}, j_{r} ; j_{d}, j_{u}}$ into separate blocks which can be separately diagonalized, implying similar block structure of the $V$ matrices as well, splitting the $\hat{M}_{\mu}$ operators defined in (73) into different sets.

First, consider the so-called zero block $\hat{B}_{0}$ in which $j_{l}=j_{r}$ as well as $j_{d}=j_{u}$. The elements of this block will be linear combinations of products of horizontal and vertical on-leg singlet projectors:

$$
\hat{B}_{0}=\left(\tau_{0}\right)_{j j ; j^{\prime} j^{\prime}}|0(j)\rangle\left\langle 0(j)|\otimes| 0\left(j^{\prime}\right)\right\rangle\left\langle 0\left(j^{\prime}\right)\right| .
$$

The $\hat{M}_{\mu}$ operators derived from this block will be diagonal in the space of singlets; the block $\left(\tau_{0}\right)_{j j ; j^{\prime} j^{\prime}}$ is a simple symmetric matrix, diagonalizable by the orthogonal block $V_{j \mu}^{(0)}$, using which we obtain the diagonal operators

$$
\hat{M}_{\mu}^{(0)}=\sum_{j} V_{j \mu}^{(0)}|0(j)\rangle\langle 0(j)| .
$$

The next blocks are responsible to $\hat{M}_{\mu}$ which are off diagonal in the singlet space. In the $U(1)$ case, for example, we will have blocks for which $j_{l}-j_{r}=j_{u}-j_{d}= \pm k$ (for any integer $k$ allowed by our tensors):

$$
\hat{B}_{ \pm k}=\left(\tau_{0}\right)_{j, j \mp k ; j^{\prime}, j^{\prime} \pm k}|0(j)\rangle\left\langle 0(j \mp k)|\otimes| 0\left(j^{\prime}\right)\right\rangle\left\langle 0\left(j^{\prime} \pm k\right)\right| .
$$

Let us choose, in our $U(1)$ example, to include one copy of each irrep $|j| \leqslant J$ ( $J$ may also be infinite). The matrix $\left(\tau_{0}\right)_{j_{l}, j_{r} ; j_{u}, j_{d}}$ will have dimension $(2 J+1)^{2}$. The zeroth block of $\hat{\tau}, B_{0}=\left(\tau_{0}\right)_{j, j k ; j^{\prime}, j^{\prime}}$, will be a $2 J+1$-dimensional matrix (since there are $2 J+1$ possible on-leg singlet states). The blocks $B_{k}=\left(\tau_{0}\right)_{j, j \mp k ; j^{\prime}, j^{\prime} \pm k}$ will each be $\left.2 J+1-|k|\right)$ dimensional (counting the number of $j$ values allowing for $j-k$ and $j+k$ values which agree with $|j| \leqslant J)$, from $k= \pm 1$ until $k= \pm 2 J-$ altogether $2 J+1$ blocks the dimensions of which add up, properly, to the right matrix dimension, $\sum_{k=-2 J}^{2 J}(2 J+1-|k|)=(2 J+1)^{2}$. Finally, since $\left(\hat{\tau}_{0}\right)_{j_{l}, j_{r} ; j_{d}, j_{u}}$ is a symmetric matrix, we obtain that $B_{k}=B_{-k}^{T}$, and write down the matrix in the block form

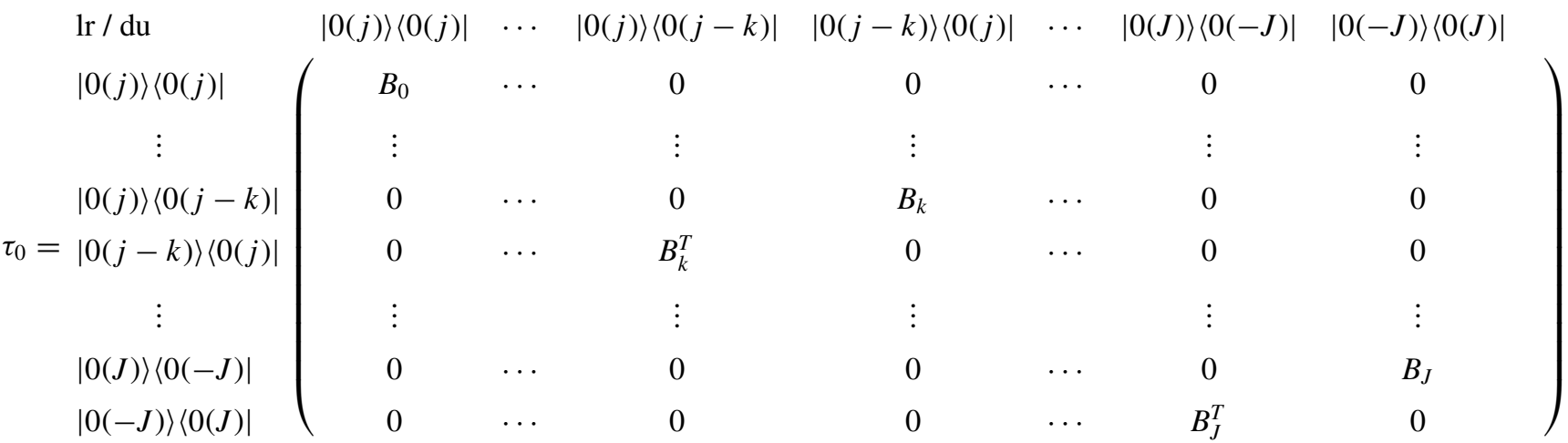

(where the headers of the rows and columns denote the type of operators they connect with). This matrix can be easily blockwise diagonalized, involving the diagonalization of $J+1$ different blocks. Similar forms can be written also for other gauge groups (later on, we will work out a detailed example for the $\mathbb{Z}_{2}$ case). 
Before moving on to the contraction of Wilson loops, we shall consider some simple illustrative cases of norm computation, regardless of the gauge group. First, assume that all the blocks but the zeroth one vanish, and, on top of that, that the zeroth block is diagonal, that is,

$$
\hat{\tau}_{0}=\sum_{j} \lambda_{j}|0(j)\rangle\langle 0(j)|\otimes| 0(j)\rangle\langle 0(j)| ;
$$

all the relevant $\hat{M}_{\mu}$ operators are projectors (the other ones do not contribute since they are associated with zero eigenvalues). Then, it is easy to see that the transfer matrix is

$$
\hat{E}=\sum_{j} \lambda_{j}^{\mathcal{N}}|0(j)\rangle\langle 0(j)|\otimes| 0(j)\rangle\langle 0(j)|\otimes \cdots \otimes| 0(j)\rangle\langle 0(j)| .
$$

Then, the eigenvectors are product vectors of the same representation, $\left\langle w_{j}\right|=\langle 0(j)| \otimes \cdots \otimes\langle 0(j)|$ with eigenvalues $\rho_{j}=\lambda_{j}^{\mathcal{N}}$, and the norm is

$$
\langle\psi \mid \psi\rangle=\operatorname{Tr}\left[\hat{E}^{\mathcal{N}}\right]=\sum_{j} \lambda_{j}^{\mathcal{N}^{2}} .
$$

Next, we keep the off-diagonal terms of the zeroth block zero, but allow for very small nonzero elements in the other blocksthat is, significantly smaller (in absolute value) than the diagonal terms of the zeroth block. If $D$ irreps participate in our state, we have $\hat{M}_{\mu}=\left|0\left(j_{\mu}\right)\right\rangle\left\langle 0\left(j_{\mu}\right)\right|$ for $\mu=1, \ldots, D$, with eigenvalues $\left|\lambda_{1}\right| \geqslant \ldots \geqslant\left|\lambda_{D}\right|>0$, while, for some $K>D$, $\left|\lambda_{\mu+1}\right| \geqslant$ $\ldots \geqslant\left|\lambda_{\mu+K}\right|>0$ and there is some $1 \leqslant L \leqslant D$ for which $\left|\lambda_{\mu+1}\right| \ll\left|\lambda_{L}\right|$. Then one may use perturbation theory to find the spectrum of $\hat{E}$. The nonperturbed part is $\sum_{\mu \leqslant L} \lambda_{\mu}^{N}\left|0\left(j_{\mu}\right)\right\rangle\left\langle 0\left(j_{\mu}\right)|\otimes| 0\left(j_{\mu}\right)\right\rangle\left\langle 0\left(j_{\mu}\right)|\otimes \cdots \otimes| 0\left(j_{\mu}\right)\right\rangle\left\langle 0\left(j_{\mu}\right)\right|$, giving rise to zerothorder eigenvectors as before, with corrections which are product vectors as well.

Now allow for nonzero weak o-diagonal elements in the zeroth block. Perturbation theory is still valid, keeping our eigenvectors close to product states along the row. In fact, as long as the diagonal terms of the zeroth block are significantly stronger (in absolute value) than the rest of the $\tau_{0}$ elements, this argument holds. As these other terms get larger and larger, the perturbative description loses its validity and the eigenvectors get farther from being product states along the row.

This may be interpreted as the lack or the presence of long-range order: the farther we are from product states along the row, the longer-ranged order we have. Since confinement has to do with disorder [45], we find here the first hint to detecting area law from the transfer operators. As we shall see later on, indeed, the closer the transfer matrix eigenvectors are to product states, the closer we are to an area law of the Wilson loop.

\section{A TALE OF TILING: CONTRACTING WILSON LOOPS}

After having computed the norms, we move further to the contraction of Wilson loop expectation values, which first requires studying further local ingredients: the flux-carrying transfer operators.

\section{A. Flux carrying transfer operators}

Consider the transfer operators associated with sites carrying a straight flux line-that is, a group element operator $U^{j}$ (or $U^{j \dagger}$ ) acting on either the horizontal or vertical direction, computed using (60):

$$
\begin{aligned}
& \left(\left[T_{\rightarrow}\right]_{M N}^{J}\right)_{l l^{\prime}, r r^{\prime} ; d d^{\prime}, u u^{\prime}}=\operatorname{Tr}_{\mathrm{phys}}\left[A_{r u l d}^{s t} U_{M N}^{s J}|s t\rangle\left\langle s^{\prime} t^{\prime}\right| \bar{A}_{r^{\prime} u^{\prime} l^{\prime} d^{\prime}}^{s^{\prime} t^{\prime}}\right]=A_{r u l d}^{s t} \bar{A}_{r^{\prime} u^{\prime} l^{\prime} d^{\prime}}^{s^{\prime} t}\left\langle s^{\prime}\left|U_{M N}^{J}\right| s\right\rangle \equiv M_{1}^{J} \\
& \left.\left(\left[T_{\uparrow}\right]_{M N}^{J}\right)_{l l^{\prime}, r r^{\prime} ; d d^{\prime}, u u^{\prime}}=\operatorname{Tr}_{\text {phys }}\left[A_{r u l d}^{s t} U_{M N}^{t J}|s t\rangle\left\langle s^{\prime} t^{\prime}\right| \bar{A}_{r^{\prime} u^{\prime} l^{\prime} d^{\prime}}^{\prime}\right]=A_{r u l d}^{s t} \bar{A}_{r^{\prime} u^{\prime} l^{\prime} d^{\prime}}^{s t^{\prime}}\left\langle t^{\prime}\left|U_{M N}^{J}\right| t\right\rangle \equiv\right]_{-1}^{J} \\
& \left(\left[T_{\leftarrow}\right]_{M N}^{J}\right)_{l l^{\prime}, r r^{\prime} ; d d^{\prime}, u u^{\prime}}=\operatorname{Tr}_{\text {phys }}\left[A_{r u l d}^{s t} U_{M N}^{s{ }^{\dagger}}|s t\rangle\left\langle s^{\prime} t^{\prime}\right| \bar{A}_{r^{\prime} u^{\prime} l^{\prime} d^{\prime}}^{s^{\prime}}\right]=A_{r u l d}^{s t} \bar{A}_{r^{\prime} u^{\prime} l^{\prime} d^{\prime}}^{s^{\prime} t}\left\langle s^{\prime}\left|U_{M N}^{J \dagger}\right| s\right\rangle \equiv \underbrace{N}_{-} \\
& \left(\left[T_{\downarrow}\right]_{M N}^{J}\right)_{l l^{\prime}, r r^{\prime} ; d d^{\prime}, u u^{\prime}}=\operatorname{Tr}_{\text {phys }}\left[A_{r u l d}^{s t} U_{M N}^{t J^{\dagger}}|s t\rangle\left\langle s^{\prime} t^{\prime}\right| \bar{A}_{r^{\prime} u^{\prime} l^{\prime} d^{\prime}}^{s^{\prime} \prime^{\prime}}\right]=A_{r u l d}^{s t} \bar{A}_{r^{\prime} u^{\prime} l^{\prime} d^{\prime}}^{s t^{\prime}}\left\langle t^{\prime}\left|U_{M N}^{J \dagger}\right| t\right\rangle \equiv \underbrace{J \mid-1}_{-1}
\end{aligned}
$$


(a)

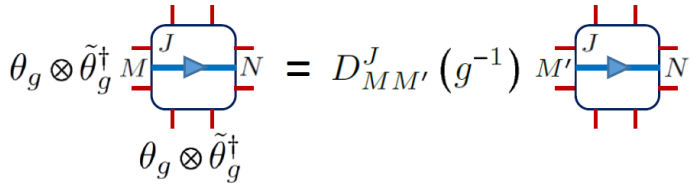

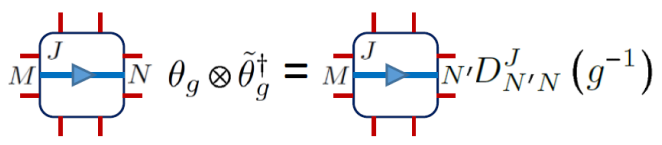

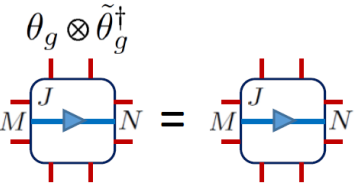

(c)

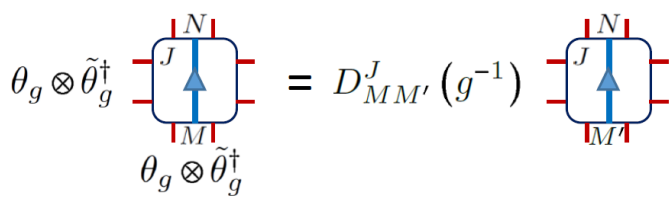

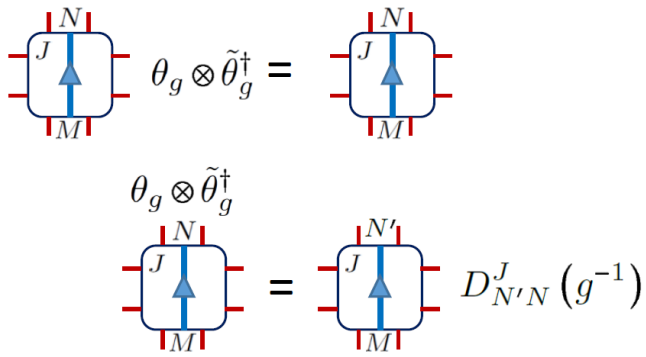

(b)

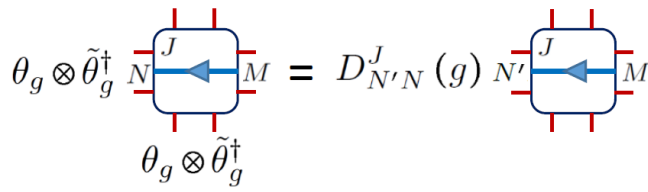
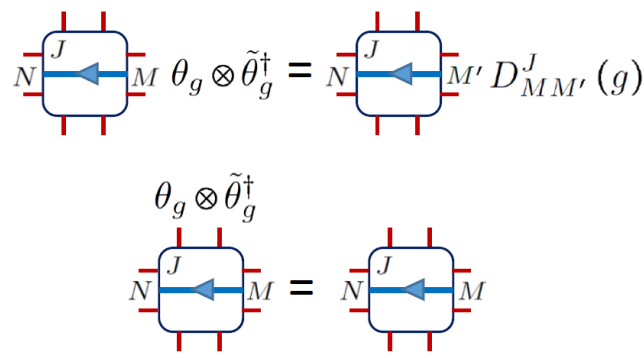

(d)
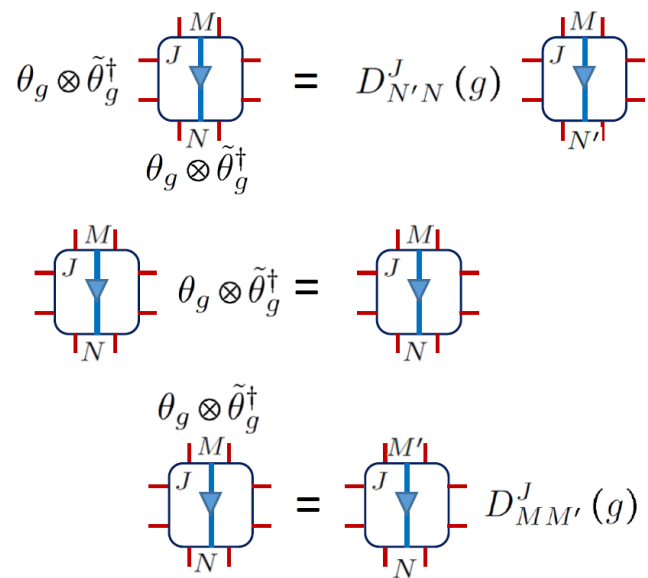

FIG. 7. Transformation rules of the straight flux carrying transfer operators: (a) $\left[\hat{T}_{\rightarrow}\right]_{M N}^{J}-$ Eq. (85), (b) $\left[\hat{T}_{\leftarrow}\right]_{M N}^{J}-$ Eq. (87), (c) $\left[\hat{T}_{\uparrow}\right]_{M N}^{J}-$ Eq. (88), and (d) $\left[\hat{T}_{\downarrow}\right]_{M N}^{J}$-Eq. (89).

Using the symmetry conditions (44) as well as the transformation properties of the group element operators (31), we obtain that for every $g \in G$

$$
\begin{aligned}
\left(\theta_{g}^{l} \otimes \tilde{\theta}_{g}^{\dagger l^{\prime}}\right) \otimes\left(\theta_{g}^{d} \otimes \tilde{\theta}_{g}^{\dagger d^{\prime}}\right)\left[\hat{T}_{\rightarrow}\right]_{M N}^{J} & =D_{M M^{\prime}}^{J}\left(g^{-1}\right)\left[\hat{T}_{\rightarrow}\right]_{M^{\prime} N}^{J}, \\
{\left[\hat{T}_{\rightarrow}\right]_{M N}^{J}\left(\theta_{g}^{r} \otimes \tilde{\theta}_{g}^{\dagger r^{\prime}}\right) } & =\left[\hat{T}_{\rightarrow}\right]_{M N^{\prime}}^{J} D_{N^{\prime} N}^{J}\left(g^{-1}\right), \\
{\left[\hat{T}_{\rightarrow}\right]_{M N}^{J}\left(\theta_{g}^{u} \otimes \tilde{\theta}_{g}^{\dagger u^{\prime}}\right) } & =\left[\hat{T}_{\rightarrow}\right]_{M N}^{J}
\end{aligned}
$$

[see Fig. 7(a)]. That is, $\left[\hat{T}_{\rightarrow}\right]_{M N}^{J}$ maps from a total $\langle J M|$ on both ingoing legs [with respect to $\left(\theta_{g}^{l} \otimes \tilde{\theta}_{g}^{\dagger l^{\prime}}\right) \otimes\left(\theta_{g}^{d} \otimes \tilde{\theta}_{g}^{\dagger d^{\prime}}\right)$ ] onto $\langle J N|$ with respect to $\left(\theta_{g}^{r} \otimes \tilde{\theta}_{g}^{\dagger r^{\prime}}\right)$ on the outgoing horizontal leg and a singlet with respect to $\left(\theta_{g}^{u} \otimes \tilde{\theta}_{g}^{\dagger u^{\prime}}\right)$ on the outgoing vertical leg [see Fig. 8(a)]. As in the flux-free case, that will have implications on the structure of the $\left[\hat{T}_{\rightarrow}\right]_{M N}^{J}$ operators.

Furthermore, the transfer operators $\left[\hat{T}_{\rightarrow}\right]_{M N}^{J}$ form a multiplet for each $J$, the elements of which are mixed by the
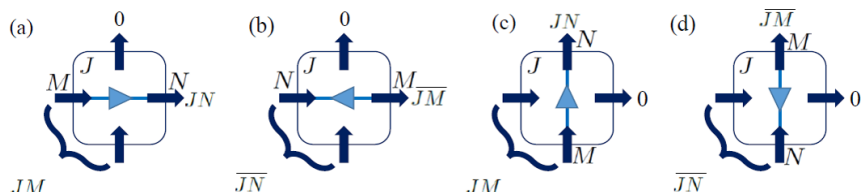

FIG. 8. The straight flux line transfer operators as maps transformations. There is no problem with that, because in the contraction of the Wilson loop we sum over the $M, N$ indices (matrix product and tracing of the $U$ matrices). As usual, in the Abelian case the multiplets are trivial and contain one operator only, allowing us to give an intuitive illustration. For example, let us consider $U(1)$ with the fundamental representation $j=1$; there, the transformations take the simple form

$$
\begin{aligned}
e^{i \phi\left(E^{l}-E^{l^{\prime}}+E^{d}-E^{d^{\prime}}\right)} \hat{T}_{\rightarrow} & =e^{-i \phi} \hat{T}_{\rightarrow}, \hat{T}_{\rightarrow} e^{i \phi\left(E^{r}-E^{r^{\prime}}\right)}=e^{-i \phi} \hat{T}_{\rightarrow}, \\
\hat{T}_{\rightarrow} e^{i \phi\left(E^{u}-E^{u^{\prime}}\right)} & =\hat{T}_{\rightarrow} .
\end{aligned}
$$

For the inverse horizontal flux line, one obtains

$$
\begin{aligned}
\left(\theta_{g}^{l} \otimes \tilde{\theta}_{g}^{\dagger l^{\prime}}\right) \otimes\left(\theta_{g}^{d} \otimes \tilde{\theta}_{g}^{\dagger d^{\prime}}\right)\left[\hat{T}_{\leftarrow}\right]_{M N}^{J} & =\left[\hat{T}_{\leftarrow}\right]_{M N^{\prime}}^{J} D_{N^{\prime} N}^{J}(g), \\
{\left[\hat{T}_{\leftarrow}\right]_{M N}^{J}\left(\theta_{g}^{r} \otimes \tilde{\theta}_{g}^{\dagger r^{\prime}}\right) } & =D_{M M^{\prime}}^{J}(g)\left[\hat{T}_{\leftarrow}\right]_{M^{\prime} N}^{J}, \\
{\left[\hat{T}_{\leftarrow}\right]_{M N}^{J}\left(\theta_{g}^{u} \otimes \tilde{\theta}_{g}^{\dagger u^{\prime}}\right) } & =\left[\hat{T}_{\leftarrow}\right]_{M N}^{J}
\end{aligned}
$$

[see Fig. 7(b)] - the difference from the right going flux is not very big, and has to do mainly on the opposite flux orientation: $g$ instead of $g^{-1}$ appears in the transformation, and the beginning index $M$ is now associated with the right side rather than the left (similarly, $N$ with the left rather than the right), since the flux goes backwards. This corresponds to transposition, and since the representations are unitary $D_{n m}^{j}(g)=\overline{D_{m n}^{j}\left(g^{-1}\right)}$-i.e., the conjugate representation $J$. As 
a result, we denote the input of both legs as $\langle\overline{J N}|$ and the output of the right leg as $\langle\overline{J M}|$ - vectors with a conjugate transformation rule [see Fig. 8(b)].

In the vertical direction, we have

$$
\begin{aligned}
\left(\theta_{g}^{l} \otimes \tilde{\theta}_{g}^{\dagger l^{\prime}}\right) \otimes & \left(\theta_{g}^{d} \otimes \tilde{\theta}_{g}^{\dagger d^{\prime}}\right)\left[\hat{T}_{\uparrow}\right]_{M N}^{J}=D_{M M^{\prime}}^{J}\left(g^{-1}\right)\left[\hat{T}_{\uparrow}\right]_{M^{\prime} N}^{J}, \\
{\left[\hat{T}_{\uparrow}\right]_{M N}^{J}\left(\theta_{g}^{r} \otimes \tilde{\theta}_{g}^{\dagger r^{\prime}}\right) } & =\left[\hat{T}_{\uparrow}\right]_{M N}^{J}, \\
{\left[\hat{T}_{\uparrow}\right]_{M N}^{J}\left(\theta_{g}^{u} \otimes \tilde{\theta}_{g}^{\dagger u^{\prime}}\right) } & =\left[\hat{T}_{\uparrow}\right]_{M N^{\prime}}^{J} D_{N^{\prime} N}^{J}\left(g^{-1}\right)
\end{aligned}
$$

[Fig. 7(c)] and

$$
\begin{aligned}
\left(\theta_{g}^{l} \otimes \tilde{\theta}_{g}^{\dagger l^{\prime}}\right) \otimes & \left(\theta_{g}^{d} \otimes \tilde{\theta}_{g}^{\dagger d^{\prime}}\right)\left[\hat{T}_{\downarrow}\right]_{M N}^{J}=\left[\hat{T}_{\downarrow}\right]_{M N^{\prime}}^{J} D_{N^{\prime} N}^{J}(g), \\
{\left[\hat{T}_{\downarrow}\right]_{M N}^{J}\left(\theta_{g}^{r} \otimes \tilde{\theta}_{g}^{\dagger r^{\prime}}\right) } & =\left[\hat{T}_{\downarrow}\right]_{M N}^{J}, \\
{\left[\hat{T}_{\downarrow}\right]_{M N}^{J}\left(\theta_{g}^{u} \otimes \tilde{\theta}_{g}^{\dagger u^{\prime}}\right) } & =D_{M M^{\prime}}^{J}(g)\left[\hat{T}_{\downarrow}\right]_{M^{\prime} N}^{J}
\end{aligned}
$$

[Fig. 7(d)]. The input/output pictures, when looking at these operators as maps, are shown in Figs. 8(c) and 8(d). Note that when plugging the trivial representation into any of the results for straight flux lines, that is, $J=M=N=0, \hat{T}$ is obtained.

There are many other options to consider, in which flux line(s) go through a site. Here we only look at the ones required for our counterclockwise Wilson loop contraction, which implies naively that four further types of transfer operators, for the corners, are required. However, we only need one, as we shall see shortly when tiling the loop:

$$
\begin{aligned}
& \left(\left[\hat{T}_{\searrow}\right]_{M N}^{J}\right)_{l l^{\prime}, r r^{\prime} ; d d^{\prime}, u u^{\prime}} \\
& =\operatorname{Tr}_{\text {phys }}\left[A_{r u l d}^{s t} U_{M K}^{t J^{\dagger}} U_{K N}^{s J}|s t\rangle\left\langle s^{\prime} t^{\prime}\right| \bar{A}_{r^{\prime} u^{\prime} l^{\prime} d^{\prime}}^{s^{\prime}}\right] \\
& \left.=A_{r u l d}^{s t} \bar{A}_{r^{\prime} u^{\prime} l^{\prime} d^{\prime}}^{s^{\prime}}\left\langle t^{\prime}\left|U_{M K}^{J \dagger}\right| t\right\rangle\left\langle s^{\prime}\left|U_{K N}^{J}\right| s\right\rangle \equiv\right]_{N}^{J}
\end{aligned}
$$

Its transformation properties may be similarly derived, resulting in

$$
\begin{aligned}
\left(\theta_{g}^{l} \otimes \tilde{\theta}_{g}^{\dagger l^{\prime}}\right) \otimes & \left(\theta_{g}^{d} \otimes \tilde{\theta}_{g}^{\dagger d^{\prime}}\right)\left[\hat{T}_{\searrow}\right]_{M N}^{J}=\left[\hat{T}_{\searrow}\right]_{M N}^{J}, \\
& {\left[\hat{T}_{\searrow}\right]_{M N}^{J}\left(\theta_{g}^{r} \otimes \tilde{\theta}_{g}^{\dagger r^{\prime}}\right)=\left[\hat{T}_{\searrow}\right]_{M N^{\prime}}^{J} D_{N^{\prime} N}^{J}\left(g^{-1}\right), } \\
& {\left[\hat{T}_{\searrow}\right]_{M N}^{J}\left(\theta_{g}^{u} \otimes \tilde{\theta}_{g}^{\dagger u^{\prime}}\right)=D_{M M^{\prime}}^{J}(g)\left[\hat{T}_{\searrow}\right]_{M^{\prime} N}^{J} ; }
\end{aligned}
$$

the ingoing legs form a combined singlet, while both the outgoing legs, separately, belong to the $J$ representation (one regular, one conjugate)—see Fig. 9.

\section{B. Tiling the loop and projecting onto smaller spaces}

Do we need to use all the elements of the transfer operators for the Wilson loop contraction? The answer is no; we can ignore some of them in the computation, while tiling the different building blocks together, thanks to the local symmetry and the special properties it enforces on the states and the transfer operators, just like we did in the case of the norm. As discussed, each of the local transfer operators used for the contraction, either with or without flux, can be seen as a map between the two ingoing legs to the two outgoing ones. While the ingoing legs form together a multiplet vector of the group, the output is a product of two separate multiplet vectors on the two outgoing legs [see Figs. 6(b), 8, and 9(b)]. The numerator of the Wilson loop expectation value requires a particular tiling of the transfer operators, closing the loop. Since the output to each direction forms a multiplet vector, this will also be the input of the neighboring transfer operators in the outgoing directions, and we can restrict all our transfer operators by cutting off all the input options that could not be realized within the Wilson loop tiling. This is done in a very similar way to what we did in the norm computation, where we defined $\hat{\tau}_{0}(67)$ instead of $\hat{T}$.

Since our system is translationally invariant, let us identify the lower left corner of the loop with the lower left corner of our system. Let us consider the numerator of the expectation value of the Wilson loop:
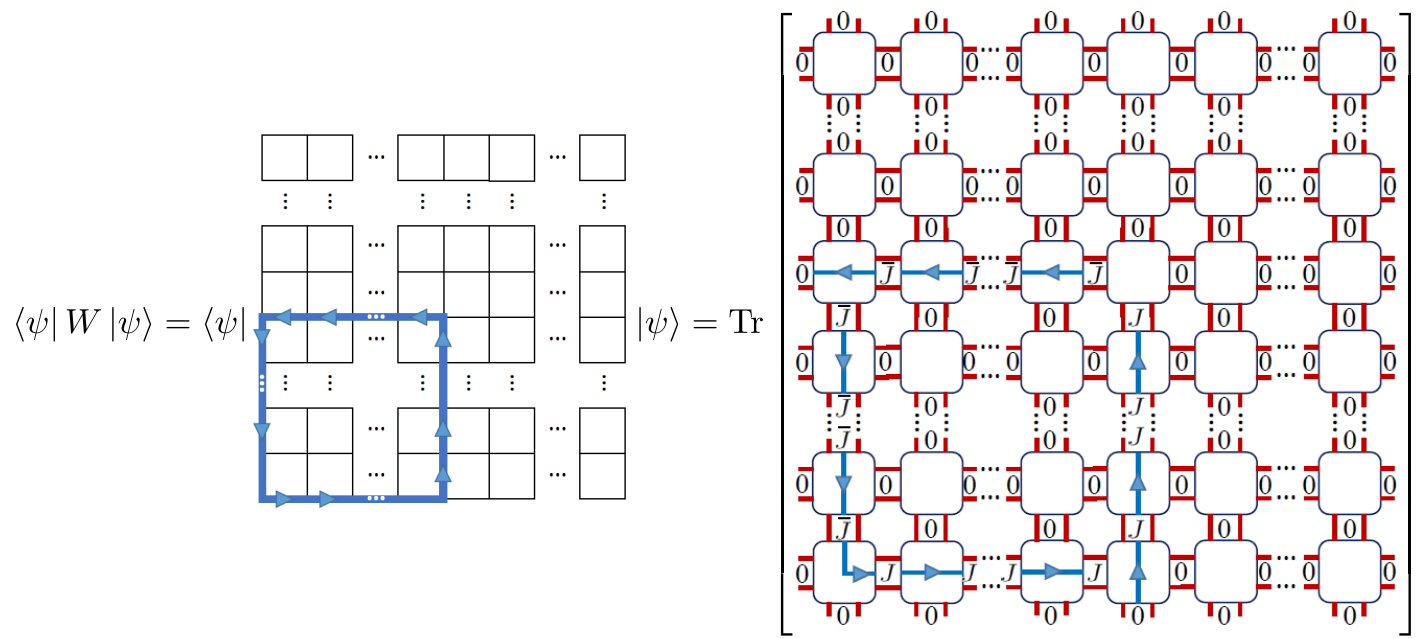
(a)

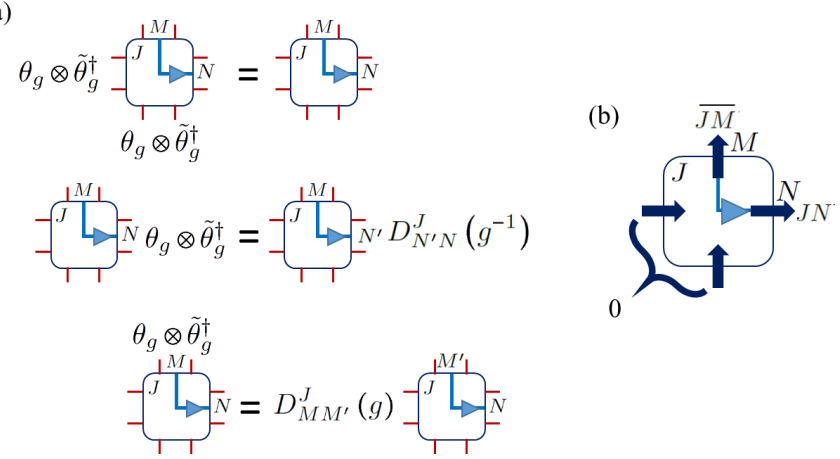

FIG. 9. The lower left corner transfer operator: (a) transformation rules and (b) as a map.

where the trace is on both directions, assuming periodic boundaries (similar results may be easily derived for open boundary conditions); the $J, M, N$ indices of the flux-carrying transfer operators have been omitted for simplicity, but it is assumed that they all carry the same irrep $J$ (otherwise it would make no physical sense) and that the $M, N$ indices are properly connected and summed over along the loop. Using the mapping properties summarized in Figs. 6(b), 8, and 9(b), we can write on each of the outgoing legs its output representation $-0, J$, or $\bar{J}$ for the conjugate representations used in the backwards fluxes cases. This immediately determines onto which inputs the transfer operators should be projected. Note that the lower right and both upper corners do not seem right in the equation above; nevertheless these are the right ingredients to be used, as explained below.

The tiling is composed of the following ingredients.

(1) Outside of the loop and within it, on sites through which no flux lines pass, we use the flux-free transfer operator $\hat{T}$. They only receive zero as inputs, and thus may be replaced by $\hat{\tau}_{0}$ from Eq. (66) in all these places.

(2) On the lower left corner, we use the $\left[\hat{T}_{\searrow}\right]^{J}$, which, thanks to receiving zero inputs on both directions from $\hat{\tau}_{0}$ operators, may be replaced by

$$
\left[\hat{\tau}_{\searrow}\right]_{M N}^{J}=\Pi_{0} \otimes \Pi_{0}\left[\hat{T}_{\searrow}\right]_{M N}^{J} \equiv \bigsqcup^{J}
$$

(3) Along the lower edge, until the next corner, we use $\left[\hat{T}_{\rightarrow}\right]^{J}$. As the input of these operators is $J$ from the left and zero from below, they may be replaced by

$$
\left[\hat{\tau}_{\rightarrow}\right]_{M N}^{J}=\Pi_{J M} \otimes \Pi_{0}\left[\hat{T}_{\rightarrow}\right]_{M N}^{J} \equiv M \longrightarrow{ }_{N}^{J}
$$

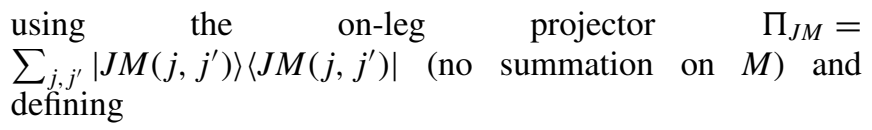

$$
\left|J M\left(j, j^{\prime}\right)\right\rangle=\left\langle J M j m \mid j^{\prime} m^{\prime}\right\rangle\left|j m j^{\prime} m^{\prime}\right\rangle .
$$

(4) When turning upwards, in the lower right corner, we use $\left[\hat{T}_{\uparrow}\right]^{J}$ : our tensors only contain physical degrees of freedom on the outgoing links; at this site the only physical leg carrying flux is the one pointing upwards and therefore this is the relevant transfer operator. Its input allows us to restrict it to

$$
\left[\hat{\tau}_{\nearrow}\right]_{M N}^{J}=\Pi_{J M} \otimes \Pi_{0}\left[\hat{T}_{\uparrow}\right]_{M N}^{J} \equiv{ }_{M}{ }^{J} \stackrel{S}{S}^{N}
$$

(5) We go along with $\left[\hat{T}_{\uparrow}\right]^{J}$ all the way up until the top right corner, but with different input, introducing

$$
\left[\hat{\tau}_{\uparrow}\right]_{M N}^{J}=\left.\Pi_{0} \otimes \Pi_{J M}\left[\hat{T}_{\uparrow}\right]_{M N}^{J} \equiv\right|_{M} ^{J}
$$

(6) At the upper right corner, the fluxes only come from the ingoing legs, and therefore the relevant transfer operator is once again $\hat{T}$, projected this time onto

$$
\left[\hat{\tau}_{\nwarrow}\right]_{M N}^{J}=\Pi_{\overline{J N}} \otimes \Pi_{J M} \hat{T} \equiv{ }^{N} \overbrace{M}^{J}
$$

where $\Pi_{\overline{J N}}=\sum_{j, j^{\prime}}\left|\overline{J N}\left(j, j^{\prime}\right)\right\rangle\left\langle\overline{J N}\left(j, j^{\prime}\right)\right|$ (no summation on $N)$ and

$$
\left|\overline{J M}\left(j, j^{\prime}\right)\right\rangle=\left\langle J M j^{\prime} m^{\prime} \mid j m\right\rangle\left|j m j^{\prime} m^{\prime}\right\rangle
$$

(7) All the way to the left we proceed with $\left[\hat{T}_{\leftarrow}\right]^{J}$ along the upper edge. Until the next corner, and without including it, it can be replaced by

$$
\left[\hat{\tau}_{\leftarrow}\right]_{M N}^{J}=\Pi_{\overline{J N}} \otimes \Pi_{0}\left[\hat{T}_{\leftarrow}\right]_{M N}^{J} \equiv N \square{ }^{J}
$$

(8) At the upper left corner we still use $\left[\hat{T}_{\leftarrow}\right]^{J}$ but with different inputs, projecting it to

$$
\left[\hat{\tau}_{\swarrow}\right]_{M N}^{J}=\Pi_{0} \otimes \Pi_{\overline{J N}}\left[\hat{T}_{\leftarrow}\right]_{M N}^{J} \equiv \underbrace{J}_{N}{ }^{M}
$$

(9) Finally, we go down with $\left[\hat{T}_{\downarrow}\right]^{J}$ all the way to the starting point, restricting it to

$$
\left[\hat{\tau}_{\downarrow}\right]_{M N}^{J}=\left.\Pi_{0} \otimes \Pi_{\overline{J N}}\left[\hat{T}_{\downarrow}\right]_{M N}^{J} \equiv\right|_{N} ^{J}
$$

Just like in the case of $\hat{\tau}_{0}$ compared with $\hat{T}$, these newly introduced operators contain less tensor elements and simplify 
the contraction of the Wilson loop:

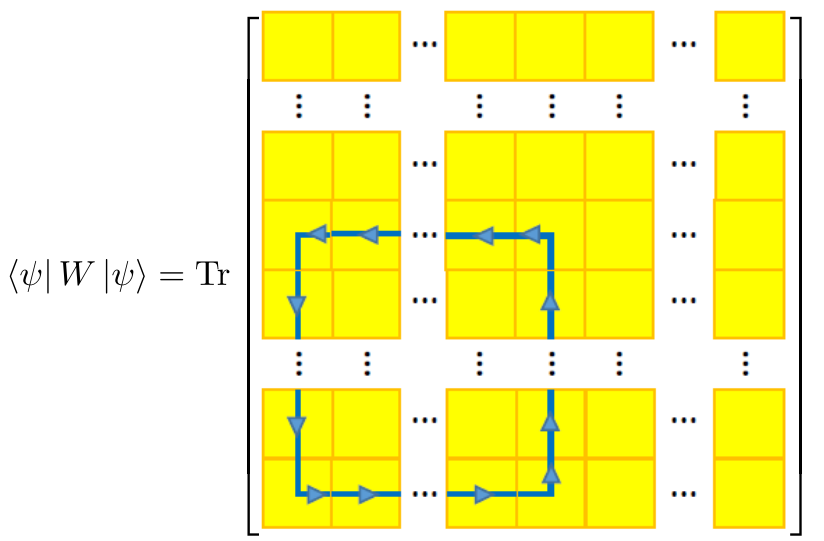

(103)

\section{Decay of Wilson loops: Is an area law possible?}

Now we have all the ingredients required for the computation of a Wilson loop the dimensions of which are $R_{1} \times R_{2}$, and compute it using row transfer matrices, by contracting first in the horizontal direction, within an $\mathcal{N} \times \mathcal{N}$ system with periodic boundary conditions (torus).

We denote the transfer matrix corresponding to the first row we contract (the one containing the lower edge of the loop) by

$$
\begin{aligned}
& {\left[\hat{E}_{b}\right]_{M N}^{J}(R) \equiv \stackrel{M}{\longrightarrow} \longrightarrow \stackrel{N}{W}_{E_{b}(R)}}
\end{aligned}
$$

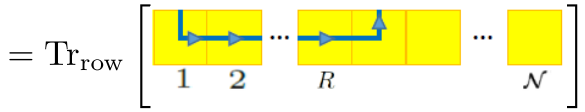

on top of it, there will be $R_{2}-1$ rows with parallel vertical flux lines, represented by

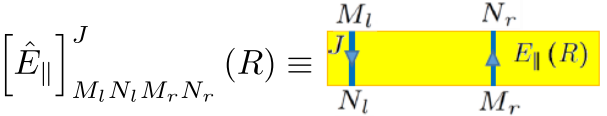

$$
\begin{aligned}
& =\operatorname{Tr}_{\text {row }}\left[\begin{array}{lll|l|l|l|l|l}
\mathbf{1} & & \cdots & & \mathbf{1} & & \cdots & \\
\hline 1 & 2 & & R & & & & \mathcal{N}
\end{array}\right]
\end{aligned}
$$

the top of the loop is represented the row transfer matrix we define by

$$
\begin{aligned}
& {\left[\hat{E}_{t}\right]_{M N}^{J}(R) \equiv{\stackrel{J}{J} \prod_{M}}^{E_{t}(R)}}
\end{aligned}
$$

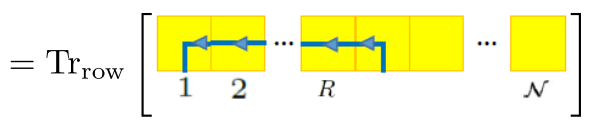

and all the remaining rows simply contribute $\hat{E}$ (we omit the $J, M, N$ indices for simplicity, assuming some given $J$ for the Wilson loop, and implicitly contracting over the $M, N$ indices). The expectation value of the Wilson loop may then be written as

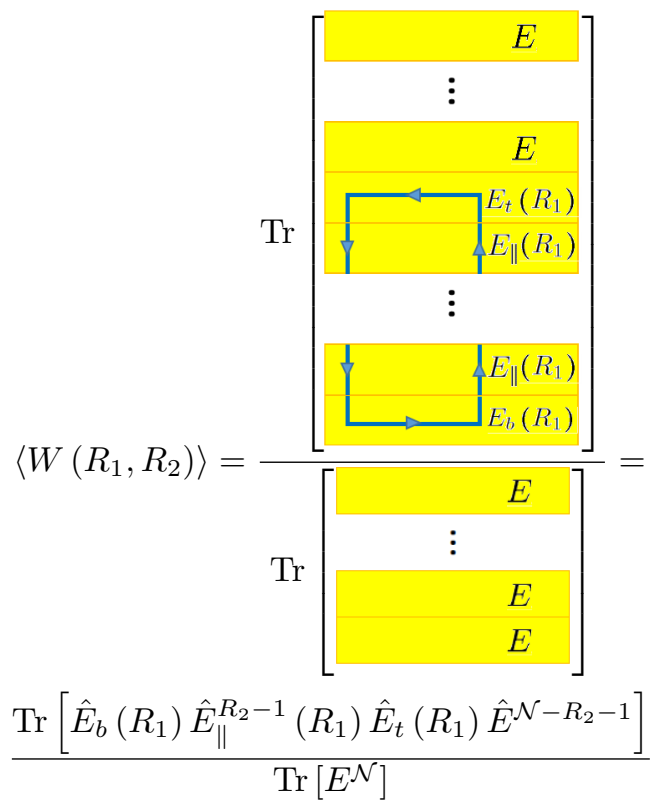

It is very similar to the MPS expression used for computing correlation functions (55) with one major difference. Due to the local symmetry, in between the two rows closing the loop, we need to use a different transfer matrix, $\hat{E}_{\|}$: the long-range decay properties depend now on two different transfer matrices, instead of one.

As stated in the beginning of this subsection, we have omitted the $M, N$ indices and we assume implicit summation over them when contracting the loop. The Wilson loop contraction consists of the contraction of $2\left(R_{1}+R_{2}\right)$ indices, each taking $\operatorname{dim}(J)$ values - naively speaking, we would have to consider $\operatorname{dim}^{2\left(R_{1}+R_{2}\right)}(J)$ different contractions; however, the singular values are independent of these indices and depend only on the irrep $J$. Thanks to this symmetry, all the $\operatorname{dim}^{2\left(R_{1}+R_{2}\right)}(J)$ are equal, so it is enough to make one choice of the indices and multiply the result by $\operatorname{dim}^{2\left(R_{1}+R_{2}\right)}(J)$. This will be a perimeterlaw term, however in the presence of an area law term it will not contribute in the large loop limit. Hence we focus below on computing for one particular choice of the indices.

Consider the diagonalization of the two transfer matrices which matter for the long-range properties:

$$
\begin{aligned}
\hat{E} & =\sum_{i} \rho_{i}\left|v_{i}\right\rangle\left\langle w_{i}\right|, \\
\hat{E}_{\|}(R) & =\sum_{i} \rho_{i}^{\prime}(R)\left|v_{i}^{\prime}(R)\right\rangle\left\langle w_{i}^{\prime}(R)\right| .
\end{aligned}
$$

Once again, we sort the eigenvalues in decreasing order, but in this case we do not care if the highest one is degenerate (but assume the existence of a spectral gap): for some integers $K, K^{\prime} \geqslant 1,\left|\rho_{1}\right|=\ldots=\left|\rho_{K}\right|>\left|\rho_{K+1}\right| \geqslant\left|\rho_{K+2}\right| \geqslant \ldots$ and $\left|\rho_{1}^{\prime}(R)\right|=\ldots=\left|\rho_{K^{\prime}}^{\prime}(R)\right|>\left|\rho_{K^{\prime}+1}^{\prime}(R)\right| \geqslant\left|\rho_{K^{\prime}+2}^{\prime}(R)\right| \geqslant \ldots$. 
Let us use this to compute the expectation value of the Wilson loop (107) in the thermodynamic limit $\mathcal{N} \gg R_{2}$ :

$$
\begin{aligned}
\left\langle W\left(R_{1}, R_{2}\right)\right\rangle & =\operatorname{dim}^{2\left(R_{1}+R_{2}\right)}(J) \frac{\rho_{1}^{N-R_{2}-1} \operatorname{Tr}\left\{\left[\sum_{i=1}^{K}\left|v_{i}\right\rangle\left\langle w_{i}\left|+\sum_{i>K}\left(\frac{\rho_{i}}{\rho_{1}}\right)^{N-R_{2}-1}\right| v_{i}\right\rangle\left\langle w_{i}\right|\right] E_{b}\left(R_{1}\right) E_{\|}^{R_{2}-1}\left(R_{1}\right) E_{t}\left(R_{1}\right)\right\}}{\rho_{1}^{N} \operatorname{Tr}\left[\sum_{i=1}^{K}\left|v_{i}\right\rangle\left\langle w_{i}\left|+\sum_{i>K}\left(\frac{\rho_{i}}{\rho_{1}}\right)^{\mathcal{N}}\right| v_{i}\right\rangle\left\langle w_{i}\right|\right]} \\
& \rightarrow \frac{\operatorname{dim}^{2\left(R_{1}+R_{2}\right)}(J)}{K \rho_{1}^{R_{2}+1}} \sum_{i=1}^{K}\left\langle w_{i}\left|\hat{E}_{b}\left(R_{1}\right) \hat{E}_{\|}^{R_{2}-1}\left(R_{1}\right) \hat{E}_{t}\left(R_{1}\right)\right| v_{i}\right\rangle
\end{aligned}
$$

(we assumed that $\rho_{1}=\ldots=\rho_{K}$; the generalization for the case of different phases is straightforward).

We further assume that the loop is large, that is, $R_{1}, R_{2} \gg 1$, allowing us to perform a similar simplification for $E_{\|}$, and obtain that in the thermodynamic limit, for large loops,

$$
\left\langle W\left(R_{1}, R_{2}\right)\right\rangle \rightarrow \operatorname{dim}^{2\left(R_{1}+R_{2}\right)}(J) \frac{\rho_{1}^{\prime R_{2}-1}\left(R_{1}\right)}{K \rho_{1}^{R_{2}+1}} \sum_{i=1}^{K} \sum_{j=1}^{K^{\prime}}\left\langle w_{i}\left|\hat{E}_{b}\left(R_{1}\right)\right| v_{j}^{\prime}\left(R_{1}\right)\right\rangle\left\langle w_{j}^{\prime}\left(R_{1}\right)\left|\hat{E}_{t}\left(R_{1}\right)\right| v_{i}\right\rangle
$$

[this holds only if $\sum_{i=1}^{K} \sum_{j=1}^{K^{\prime}}\left\langle w_{i}\left|\hat{E}_{b}\left(R_{1}\right)\right| v_{j}^{\prime}\left(R_{1}\right)\right\rangle\left\langle w_{j}^{\prime}\left(R_{1}\right)\left|\hat{E}_{t}\left(R_{1}\right)\right| v_{i}\right\rangle \neq 0$; if this condition is not fulfilled, the vectors $\left|v_{j}^{\prime}\right\rangle$ and $\left\langle w_{i}^{\prime}\right|$ should not be seen as those corresponding to the highest eigenvalues, but rather as those with the highest eigenvalues for which this condition is satisfied. We assumed here that $\rho_{1}^{\prime}=\ldots=\rho_{K^{\prime}}^{\prime}$; the generalization for the case of different phases is straightforward].

Assuming rotational invariance, we could repeat the same procedure by contracting the columns first, to obtain

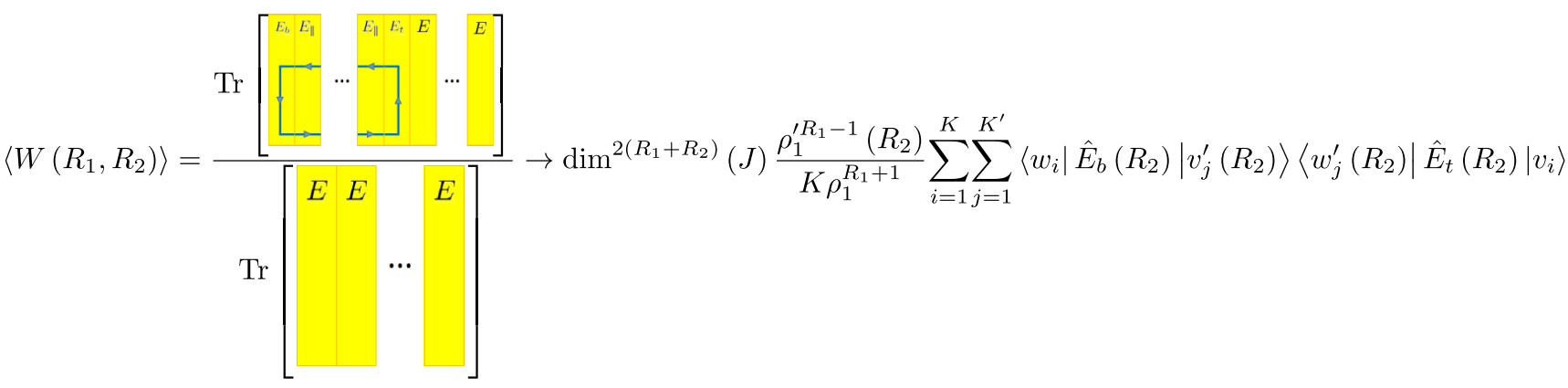

Both expressions must be equal; therefore, we deduce that

$$
\rho_{1}^{\prime R_{2}-1}\left(R_{1}\right) \sum_{i=1}^{K} \sum_{j=1}^{K^{\prime}}\left\langle w_{i}\left|\hat{E}_{b}\left(R_{1}\right)\right| v_{j}^{\prime}\left(R_{1}\right)\right\rangle\left\langle w_{j}^{\prime}\left(R_{1}\right)\left|\hat{E}_{t}\left(R_{1}\right)\right| v_{i}\right\rangle \propto \frac{1}{\rho_{1}^{R_{1}+1}} .
$$

But the more interesting question is whether $\partial \rho_{1}^{\prime}(R) / \partial R=0$ or not. If the largest eigenvalue of $\hat{E}_{\|}(R)$ does not depend on $R$, we obtain that

$$
\left\langle W\left(R_{1}, R_{2}\right)\right\rangle \rightarrow \tilde{C}\left(\operatorname{dim}^{2}(J) \frac{\rho_{1}^{\prime}}{\rho_{1}}\right)^{R_{1}+R_{2}}
$$

with some constant $\tilde{C}$ : perimeter law decay of the Wilson loop (unless $\rho_{1}=\rho_{1}^{\prime}$ ). On the other hand, an area law is possible if

$$
\rho_{1}^{\prime}(R) \sim \Gamma e^{-\kappa R}
$$

with $\kappa>0$. Let us plug this expression into (111) and (112). We will obtain the equation

$$
\begin{aligned}
\left\langle W\left(R_{1}, R_{2}\right)\right\rangle & \rightarrow \frac{\operatorname{dim}^{2\left(R_{1}+R_{2}\right)}(J)}{K \Gamma \rho_{1}}\left(\frac{\Gamma}{\rho_{1} e^{-\kappa}}\right)^{R_{1}} e^{-\kappa R_{1} R_{2}} \sum_{i=1}^{K} \sum_{j=1}^{K^{\prime}}\left\langle w_{i}\left|\hat{E}_{b}\left(R_{2}\right)\right| v_{j}^{\prime}\left(R_{2}\right)\right\rangle\left\langle w_{j}^{\prime}\left(R_{2}\right)\left|\hat{E}_{t}\left(R_{2}\right)\right| v_{i}\right\rangle \\
& =\frac{\operatorname{dim}^{2\left(R_{1}+R_{2}\right)}(J)}{K \Gamma \rho_{1}}\left(\frac{\Gamma}{\rho_{1} e^{-\kappa}}\right)^{R_{2}} e^{-\kappa R_{1} R_{2}} \sum_{i=1}^{K} \sum_{j=1}^{K^{\prime}}\left\langle w_{i}\left|\hat{E}_{b}\left(R_{1}\right)\right| v_{j}^{\prime}\left(R_{1}\right)\right\rangle\left\langle w_{j}^{\prime}\left(R_{1}\right)\left|\hat{E}_{t}\left(R_{1}\right)\right| v_{i}\right\rangle .
\end{aligned}
$$

Rotation invariance guarantees that

$$
\sum_{i=1}^{K} \sum_{j=1}^{K^{\prime}}\left\langle w_{i}\left|\hat{E}_{b}(R)\right| v_{j}^{\prime}(R)\right\rangle\left\langle w_{j}^{\prime}(R)\left|\hat{E}_{t}(R)\right| v_{i}\right\rangle \sim C\left(\frac{\Gamma}{\rho_{1} e^{-\kappa}}\right)^{R}
$$


for some constant $C$, and we obtain, finally, for large Wilson loops, that if $\rho_{1}^{\prime}(R) \sim \Gamma e^{-\kappa R}$

$$
\left\langle W\left(R_{1}, R_{2}\right)\right\rangle \rightarrow \frac{C}{K \Gamma \rho_{1}}\left(\frac{\Gamma \operatorname{dim}^{2}(J)}{\rho_{1} e^{-\kappa}}\right)^{R_{1}+R_{2}} e^{-\kappa R_{1} R_{2}},
$$

exactly the same form of (39), with $W_{0}=\frac{C}{K \Gamma \rho_{1}}, \kappa_{A}=\kappa$, and $\kappa_{P}=\log \left(\frac{\rho_{1}}{\Gamma \operatorname{dim}^{2}(J)}\right)-\kappa$.

Therefore, we conclude that a perimeter law will be obtained if the largest relevant (in terms of accessible through $\hat{E}_{b}$ and $\hat{E}_{t}$ ) eigenvalue of $\hat{E}_{\|}(R)$ is independent of $R$; an area law is possible if it depends on $R$ exponentially. Why only possible? To see why this condition is necessary but not sufficient for the area law to hold, let us consider the following scenario.

Previously, we made the assumption that the eigenvectors of the flux-free transfer matrix should be close to product vectors in order to make an area law possible. We also know that the expectation value of the Wilson loop depends on the zeroth flux transfer operators $\hat{\tau}_{0}$ inside and outside the loop, and some other, flux-carrying transfer operators along the loop. Let us assume that we are, indeed, in a scenario in which the eigenvalues of the transfer matrix are close to product states. Denote as usual the highest eigenvalue of the transfer operator by $\lambda_{1}$. Then the norm, for a large system, will roughly scale as $\lambda_{1}^{\mathcal{N}^{2}}$ : each site contributes a single power of $\lambda_{1}$. This is the denominator of the expectation value formula. In the numerator, we will have a contribution of $\lambda_{1}$ for each site outside the loop; within the loop, it depends.

If the flux carrying transfer operators along the loop take us from the singlet subspace corresponding to $\lambda_{1}$ to that of another eigenvalue - denote it by $\lambda^{\prime}$-we will have a contribution of $\lambda^{\prime}$ for each of the sites within the loop, and the Wilson loop's expectation value will scale as $\left(\lambda^{\prime} / \lambda_{1}\right)^{A}$ where $A$ is the area of the loop $\left[E_{\|}(R) \propto \lambda^{\prime R}\right]$. However, if the flux carrying transfer operators do not take us to another singlet subspace with a different eigenvalue, we will not have an area dependent contribution. In this case, the largest eigenvalue of $\hat{E}_{\|}(R)$ depends exponentially on $R$ (through $\lambda^{\prime R}$ ) but an area law is not obtained, which shows us why this condition is necessary but not sufficient.

On the other hand, if the eigenvectors of $\hat{E}$ are far from product vectors, which means they are governed by some collective, long-range effect, we cannot have area-dependent contributions at all.

\section{ILLUSTRATION: THE $\mathbb{Z}_{2}$ CASE}

To conclude and illustrate our discussion, we will show an explicit example, where the gauge group is $\mathbb{Z}_{2}$. In this case, the group Hilbert space on each link is two dimensional, with representations labeled by $j=+,-$, which can be simply seen as spins. The group element operators are Hermitian, $U=U^{\dagger}=X$, and invert the spin,

$$
X| \pm\rangle=|\mp\rangle,
$$

and the group operations $\Theta$ (no difference between left and right in Abelian groups) are the identity operator as well as

$$
Z| \pm\rangle= \pm| \pm\rangle
$$

Gauge transformations are given by

$$
\hat{\Theta}(\mathbf{x})=Z(\mathbf{x}, 1) Z(\mathbf{x}, 2) Z\left(\mathbf{x}-\hat{\mathbf{e}}_{1}, 1\right) Z\left(\mathbf{x}-\hat{\mathbf{e}}_{1}, 2\right) .
$$

We would like to consider the most general PEPS with translational and rotational invariance, with physical spaces containing all the irreps and virtual ones containing a single copy of each irrep (minimal construction-as explained above, to consider real physical scenarios one will most likely have to generalize in a straightforward manner and add more copies, as was necessary in the $\mathbb{Z}_{3}$ demonstration of [42]). Thus, the physical and virtual spaces will be the same, twodimensional spinlike spaces spanned by the representation states $| \pm\rangle$. The state will be parametrized by the tensors $A_{l r d u}^{s t}$, with $s, t, l, r, d, u= \pm$. The most general construction satisfying these conditions is given by

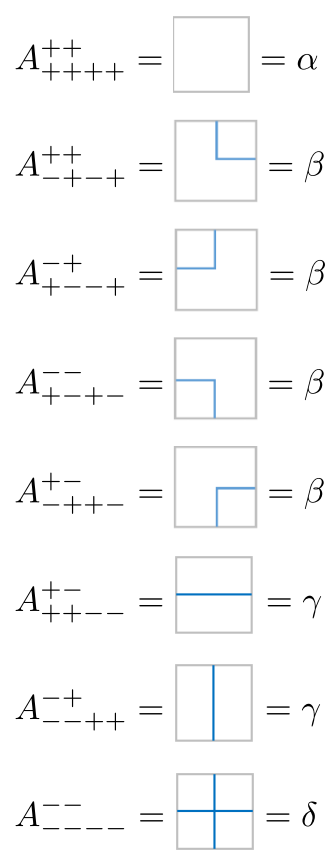

and the rest of the elements, which violate the symmetry, vanish. If we consider the $|+\rangle$ states as flux-free states, and the $|-\rangle$ as flux carrying, we can interpret $\alpha$ as the amplitude of having no fluxes going through the site, $\beta$ as the amplitude of corner flux, $\gamma$ as the amplitude of straight line fluxes, and $\delta$ as the amplitude of two intersecting flux lines.

Here we will be interested in the properties of the transfer operators constructed for such states, and the computation of the Wilson loop expectation value.

\section{A. Transfer operators}

The transfer operator $\hat{T}$ may be simply built using (58) and (59).

Let us identify the elements of the vector space spanned by the double legs of the transfer matrix. The on-leg transformations here admit the simple form $\theta \otimes \tilde{\theta}^{\dagger}=Z \otimes Z$ for the only group element which is not the identity. Since there are two irreps, we will have two on-leg singlets,

$$
\begin{aligned}
& |0(+)\rangle=|++\rangle \equiv|\uparrow\rangle \otimes|s\rangle, \\
& |0(-)\rangle=|--\rangle \equiv|\downarrow\rangle \otimes|s\rangle,
\end{aligned}
$$


as well as two nonsinglets,

$$
\begin{aligned}
& |1(+,-)\rangle=|+-\rangle \equiv|\uparrow\rangle \otimes|n\rangle, \\
& |1(-,+)\rangle=|-+\rangle \equiv|\downarrow\rangle \otimes|n\rangle .
\end{aligned}
$$

Where the new notation introduced in the two equations above factorizes the on-leg Hilbert space into the product of two spin spaces; one detects whether the state is an on-leg singlet $(s)$ or not $(n)$ and the other labels the two states within each of these options by $\uparrow$ and $\downarrow$.

Using these states, we can write down all the relevant transfer operators and their reductions. For example,

$$
\begin{aligned}
\hat{\tau}_{0} & =|\alpha|^{2}|0(+)\rangle\langle 0(+)|\otimes| 0(+)\rangle\left\langle\left. 0(+)|+| \gamma\right|^{2}(|0(+)\rangle\langle 0(+)|\otimes| 0(-)\rangle\langle 0(-)|+| 0(-)\rangle\langle 0(-)|\otimes| 0(+)\rangle\langle 0(+)|)\right. \\
& +|\delta|^{2}|0(-)\rangle\langle 0(-)|\otimes| 0(-)\rangle\left\langle\left. 0(-)|+| \beta\right|^{2}(|0(+)\rangle\langle 0(-)|+| 0(-)\rangle\langle 0(+)|) \otimes(|0(+)\rangle\langle 0(-)|+| 0(-)\rangle\langle 0(+)|) .\right.
\end{aligned}
$$

We can simplify by writing it in the matrix form, as well as adopting the new notation introduced in (122) and (123):

$$
\begin{aligned}
& \operatorname{lr} / \mathrm{du} \quad|\uparrow\rangle\langle\uparrow|\otimes| s\rangle\langle s|\quad| \downarrow\rangle\langle\downarrow|\otimes| s\rangle\langle s|\quad| \uparrow\rangle\langle\downarrow|\otimes| s\rangle\langle s|\quad| \downarrow\rangle\langle\uparrow|\otimes| s\rangle\langle s|
\end{aligned}
$$

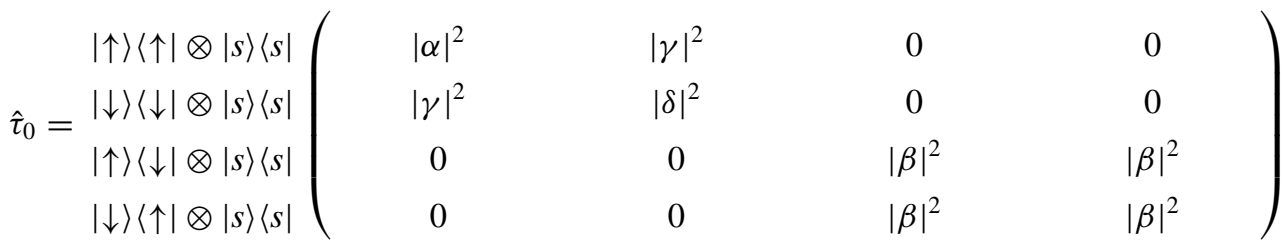

where the block structure is clearly seen; the first one is the zeroth block, mixing only projection operators. It depends on $\alpha, \gamma, \delta$ - the amplitudes for which fluxes do not change directions, and thus the representations are not changed horizontally and vertically on the state, and the on-leg singlets are not flipped on the transfer operators. The second block, where the representation/singlet changes, depends on $\beta$-the turning (corner) flux amplitude. Furthermore, as the parameter $\gamma$ has to do with straight flux lines going through the site, we expect that the larger it gets, the farther the $\hat{M}_{\mu}$ operators derived from the zeroth block are from projection operators, and the farther we are from an area law; indeed, as we see, it appears on the off-diagonal terms of the zeroth block, and when $\gamma=0$ the $\hat{M}_{\mu}$ operators of the zeroth blocks are projectors.

This matrix can be easily diagonalized as in (71), with the eigenvalues (not necessarily in descending order-this depends on the values of the parameters)

$$
\begin{aligned}
& \lambda_{1,2}=\frac{1}{2}\left(|\alpha|^{2}+|\delta|^{2} \pm \sqrt{\left(|\alpha|^{2}-|\delta|^{2}\right)^{2}+4|\gamma|^{2}}\right), \\
& \lambda_{3}=2|\beta|^{2}, \quad \lambda_{4}=0,
\end{aligned}
$$

with the diagonalizing matrix

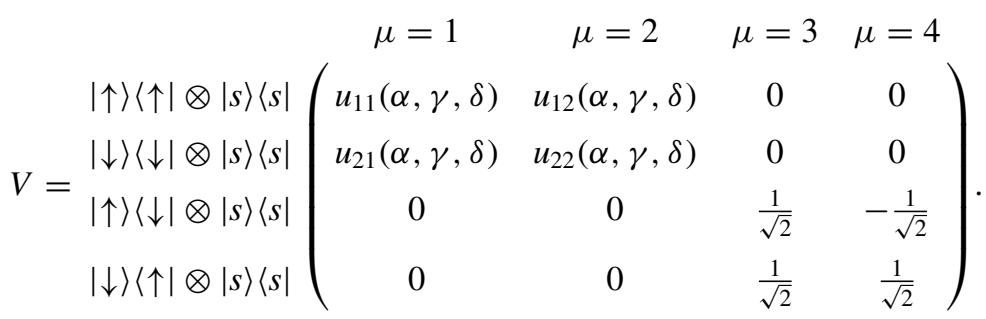

Using all that, we obtain the operators $\hat{M}_{\mu}$ as defined in (73):

$$
\begin{aligned}
& \hat{M}_{1}=\left(u_{11}(\alpha, \gamma, \delta) \Pi_{\uparrow}+u_{21}(\alpha, \gamma, \delta) \Pi_{\downarrow}\right), \\
& \hat{M}_{2}=\left(u_{12}(\alpha, \gamma, \delta) \Pi_{\uparrow}+u_{22}(\alpha, \gamma, \delta) \Pi_{\downarrow}\right), \\
& \hat{M}_{3}=\frac{1}{\sqrt{2}} \sigma_{x}, \\
& \hat{M}_{4}=-\frac{i}{\sqrt{2}} \sigma_{y},
\end{aligned}
$$

where $\Pi_{\uparrow}=|\uparrow\rangle\langle\uparrow|$ and $\Pi_{\downarrow}=|\downarrow\rangle\langle\downarrow|$. Since $V$ is orthogonal, they form an orthonormal basis as in (74). $\hat{M}_{4}$ is irrelevant, since $\lambda_{4}=0$; the $|s\rangle\langle s|$ is also irrelevant since it multiplies everything, and hence we will omit it and refer to the operators $\hat{M}_{\mu}$ as two dimensional. Note that as expected the first two ones, $\hat{M}_{1,2}$, having to do with the zeroth block, are diagonal, while the other ones are not. 
Similarly, we can compute and write down the other relevant matrices. Note that since the fluxes have no orientation in our case, $\hat{\tau}_{\rightarrow}=\hat{\tau}_{\leftarrow} \equiv \hat{\tau}_{-}$and $\hat{\tau}_{\uparrow}=\hat{\tau}_{\downarrow} \equiv \hat{\tau}_{\mid}$. We thus require only six rather than eight further matrices. The first is

$$
\begin{aligned}
& \mathrm{lr} / \mathrm{du} \quad \Pi_{\uparrow} \otimes|s\rangle\left\langle s\left|\quad \Pi_{\downarrow} \otimes\right| s\right\rangle\left\langle s\left|\quad \sigma_{+} \otimes\right| s\right\rangle\left\langle s\left|\quad \sigma_{-} \otimes\right| s\right\rangle\langle s| \\
& \hat{\tau}_{-}=\begin{array}{c}
\Pi_{\uparrow} \otimes|n\rangle\langle n| \\
\Pi_{\downarrow} \otimes|n\rangle\langle n| \\
\sigma_{+} \otimes|n\rangle\langle n| \\
\sigma_{-} \otimes|n\rangle\langle n|
\end{array}\left(\begin{array}{cccc}
\alpha \bar{\gamma} & \gamma \bar{\delta} & 0 & 0 \\
\gamma \bar{\alpha} & \delta \bar{\gamma} & 0 & 0 \\
0 & 0 & |\beta|^{2} & |\beta|^{2} \\
0 & 0 & |\beta|^{2} & |\beta|^{2}
\end{array}\right)
\end{aligned}
$$

connecting operators acting on the nonsinglet subspace in the horizontal direction with ones acting on the singlet space in the vertical one. The same block structure is apparent; the first block is a generalization of the zeroth block-still only connecting projection operators, though acting on different spaces, and the second block changes the representations. As in the $\tau_{0}$ case, the parameter $\gamma$ is the one "spoiling" the area law: all the amplitudes of $\hat{L}_{\mu}$ operators which do not change the on-leg singlet eigenvalue subspace are proportional to it. Once it is set to zero, when crossing a flux line the subspace will change.

We can formally perform a horizontal-vertical singular value decomposition and obtain an expression of the form $\hat{\tau}_{-}=$ $\sum_{\mu} \eta_{\mu} \hat{K}_{\mu} \otimes \hat{L}_{\mu}$. Since the horizontal operators act only within the nonsinglet subspace and the vertical ones only within the singlet subspace, we can represent $\hat{K}_{\mu}$ and $\hat{L}_{\mu}$ by two-dimensional matrices.

$\hat{\tau}_{\mid}$is simply obtained by transposition,

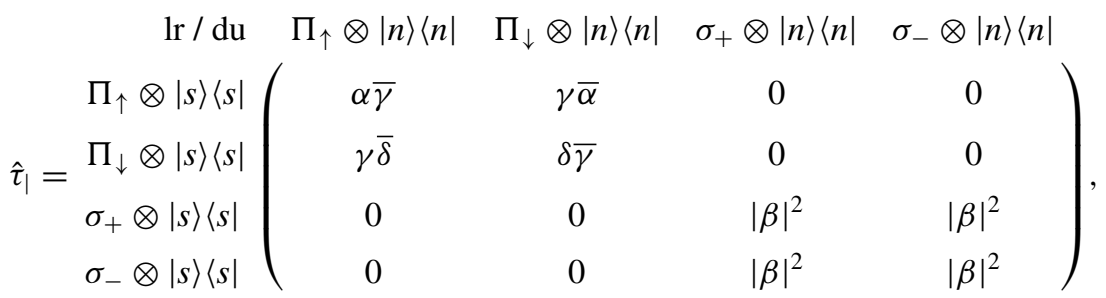

and $\hat{\tau}_{\mid}=\sum \mu \eta_{\mu} \hat{L}_{\mu} \otimes \hat{K}_{\mu}$.

Finally, let us consider the transfer operators of the four corners. We begin with the lower left corner:

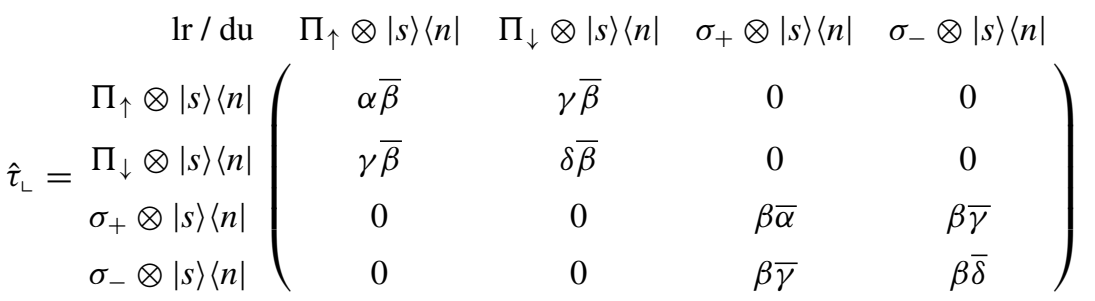

where in both dimensions we get a singlet input and obtain a nonsinglet output. Here, after performing the singular value decomposition, we will also use two-dimensional operators acting only on the "spin space" since this corner operator connects to the right $s / n$ subspaces. The other corner operators are

$$
\begin{aligned}
& \operatorname{lr} / \mathrm{du} \quad \Pi_{\uparrow} \otimes|s\rangle\left\langle n\left|\quad \Pi_{\downarrow} \otimes\right| s\right\rangle\left\langle n\left|\quad \sigma_{+} \otimes\right| s\right\rangle\left\langle n\left|\quad \sigma_{-} \otimes\right| s\right\rangle\langle n| \\
& \hat{\tau}_{\lrcorner}=\begin{array}{c}
\Pi_{\uparrow} \otimes|n\rangle\langle s| \\
\Pi_{\downarrow} \otimes|n\rangle\langle s| \\
\sigma_{+} \otimes|n\rangle\langle s| \\
\sigma_{-} \otimes|n\rangle\langle s|
\end{array}\left(\begin{array}{cccc}
\alpha \bar{\beta} & \gamma \bar{\beta} & 0 & 0 \\
\gamma \bar{\beta} & \delta \bar{\beta} & 0 & 0 \\
0 & 0 & \beta \bar{\gamma} & \beta \bar{\delta} \\
0 & 0 & \beta \bar{\alpha} & \beta \bar{\gamma}
\end{array}\right) \text {, }
\end{aligned}
$$




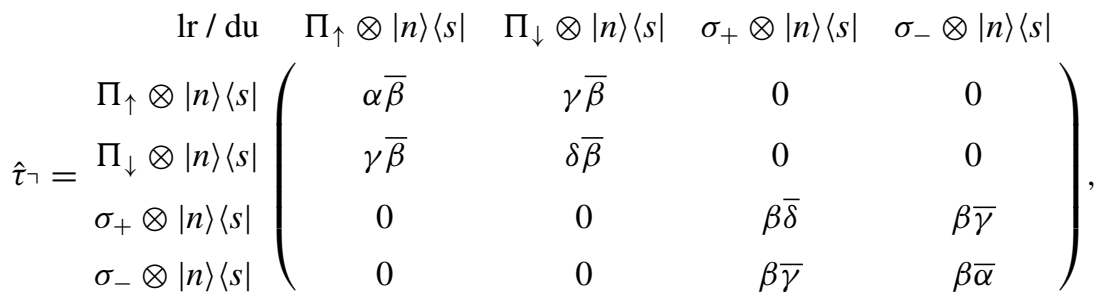

and

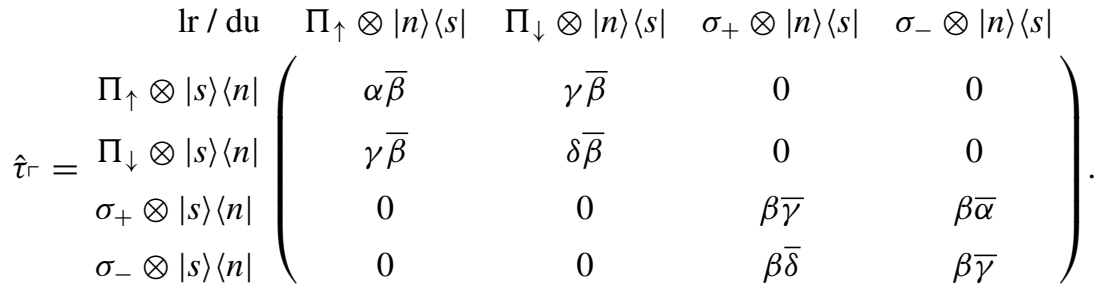

Note that all the elements of the corner operators are proportional to either $\beta$ or $\bar{\beta}$, which is expected since $\beta$ is the corner parameter, and it would be impossible to close a loop in its absence.

\section{B. Analytical example}

Let us set, for simplicity, $\gamma=0$. Consider $\tau_{0}(125)$ and the $\hat{M}_{\mu}$ operators derived from it (128). Let us set $\gamma=0$; then we simply have

$$
\hat{M}_{1}=\Pi_{\uparrow}, \quad \hat{M}_{2}=\Pi_{\downarrow}, \quad \hat{M}_{3}=\frac{1}{\sqrt{2}} \sigma_{x}, \quad \hat{M}_{4}=-\frac{i}{\sqrt{2}} \sigma_{y},
$$

as well as

$$
\lambda_{1}=|\alpha|^{2}, \quad \lambda_{2}=|\delta|^{2}, \quad \lambda_{3}=2|\beta|^{2}, \quad \lambda_{4}=0 .
$$

The choice of $\gamma=0$ sets all the zeroth block $\hat{M}_{\mu}$ operators to projectors onto orthogonal states, and the flux-free transfer matrix from (75) takes the form

$$
\begin{aligned}
\hat{E}= & |\alpha|^{2 \mathcal{N}} \Pi_{\uparrow} \otimes \cdots \otimes \Pi_{\uparrow}+|\delta|^{2 \mathcal{N}} \Pi_{\downarrow} \otimes \cdots \otimes \Pi_{\downarrow} \\
& +|\beta|^{4} \sum_{n=1}^{\mathcal{N}-1} \sum_{m}|\alpha|^{2(\mathcal{N}-n-1)}|\delta|^{2(n-1)} \Pi_{\uparrow} \otimes \cdots \otimes \Pi_{\uparrow} \otimes \underbrace{\sigma_{x}}_{m} \otimes \Pi_{\downarrow} \otimes \cdots \otimes \Pi_{\downarrow} \otimes \underbrace{\sigma_{x}}_{m+n} \otimes \Pi_{\uparrow} \otimes \cdots \otimes \Pi_{\uparrow} \\
& +|\beta|^{4} \sum_{n=1}^{\mathcal{N}-1} \sum_{m}|\delta|^{2(\mathcal{N}-n-1)}|\alpha|^{2(n-1)} \Pi_{\downarrow} \otimes \cdots \otimes \Pi_{\downarrow} \otimes \underbrace{\sigma_{x}}_{m} \otimes \Pi_{\uparrow} \otimes \cdots \otimes \Pi_{\uparrow} \otimes \underbrace{\sigma_{x}}_{m+n} \otimes \Pi_{\downarrow} \otimes \cdots \otimes \Pi_{\downarrow}+O\left(|\beta|^{8}\right) .
\end{aligned}
$$

If we further assume that $|\beta| \ll|\alpha|,|\delta|$ we find ourselves in the perturbative case discussed above, and may use perturbation theory for finding the eigenvectors of $\hat{E}$. The zeroth, unperturbed part is in the first row of (137), from which we find two approximate, zeroth-order eigenvectors,

$$
\left\langle w_{1}\right|=\langle\uparrow| \otimes \cdots \otimes\langle\uparrow|, \quad\left\langle w_{2}\right|=\langle\downarrow| \otimes \cdots \otimes\langle\downarrow|,
$$

with zeroth-order eigenvalues

$$
\rho_{1}=|\alpha|^{2 \mathcal{N}}, \quad \rho_{2}=|\delta|^{2 \mathcal{N}},
$$

which are the two highest ones. Let us set, without losing generality, $|\alpha|>|\delta|$ (one can easily invert that in the following discussion). The leading-order corrections to the eigenvalues will be second order $\left(\alpha|\beta|^{8}\right)$ and to the eigenvectors will be of the first order $\left(\propto|\beta|^{4}\right)$; we shall neglect them both. The norm of the state is then

$$
\langle\psi \mid \psi\rangle=\operatorname{Tr}\left[E^{\mathcal{N}}\right] \underset{\mathcal{N} \gg 1}{\longrightarrow} \rho_{1}^{\mathcal{N}}=|\alpha|^{2 \mathcal{N}^{2}} ;
$$

that is, the torus is tiled with $\mathcal{N}^{2}$ sites, each contributing a factor of $|\alpha|^{2}$ to the norm.

Let us now move on to the flux carrying transfer matrices. Looking at the straight flux ones $\hat{\tau}_{-}$(129) and $\hat{\tau}_{\mid}(130)$, we see that our choice of $\gamma=0$ sets the zeroth block to zero. This implies that they will flip the local incoming spins in both directions-in 
particular in the direction orthogonal to the flux; i.e., the eigenspace of $\hat{\tau}_{0}$ out of the loop will be connected to the orthogonal one within the loop, eventually to give rise to an area law, unless $|\alpha|=|\delta|$. We see that

$$
\hat{\tau}_{-}=\hat{\tau}_{\mid}=|\beta|^{2} \sigma_{x} \otimes \sigma_{x}
$$

(ignoring the $n, s$ space for the reasons explained above)_inverting the spins in the orthogonal direction to the flux lines, that is, changing indeed from the $\alpha$ to the $\delta$ sector and vice versa.

For the corners we get

$$
\begin{aligned}
& \hat{\tau}_{\llcorner}=\alpha \bar{\beta} \Pi_{\uparrow} \otimes \Pi_{\uparrow}+\delta \bar{\beta} \Pi_{\downarrow} \otimes \Pi_{\downarrow}+\beta \bar{\alpha} \sigma_{+} \otimes \sigma_{+}+\beta \bar{\delta} \sigma_{-} \otimes \sigma_{-} \equiv \sum_{\mu} \xi_{\llcorner, \mu} \hat{H}_{\llcorner, \mu} \otimes \hat{V}_{\llcorner, \mu}, \\
& \hat{\tau}_{\lrcorner}=\alpha \bar{\beta} \Pi_{\uparrow} \otimes \Pi_{\uparrow}+\delta \bar{\beta} \Pi_{\downarrow} \otimes \Pi_{\downarrow}+\beta \bar{\delta} \sigma_{+} \otimes \sigma_{-}+\beta \bar{\alpha} \sigma_{-} \otimes \sigma_{+} \equiv \sum_{\mu} \xi_{\lrcorner, \mu} \hat{H}_{\lrcorner, \mu} \otimes \hat{V}_{\lrcorner, \mu}, \\
& \hat{\tau}_{\urcorner}=\alpha \bar{\beta} \Pi_{\uparrow} \otimes \Pi_{\uparrow}+\delta \bar{\beta} \Pi_{\downarrow} \otimes \Pi_{\downarrow}+\beta \bar{\delta} \sigma_{+} \otimes \sigma_{+}+\beta \bar{\alpha} \sigma_{-} \otimes \sigma_{-} \equiv \sum_{\mu} \xi_{\urcorner, \mu} \hat{H}_{\urcorner, \mu} \otimes \hat{V}_{\urcorner, \mu}, \\
& \hat{\tau}_{\ulcorner}=\alpha \bar{\beta} \Pi_{\uparrow} \otimes \Pi_{\uparrow}+\delta \bar{\beta} \Pi_{\downarrow} \otimes \Pi_{\downarrow}+\beta \bar{\alpha} \sigma_{+} \otimes \sigma_{-}+\beta \bar{\delta} \sigma_{-} \otimes \sigma_{+} \equiv \sum_{\mu} \xi_{\ulcorner, \mu} \hat{H}_{\ulcorner, \mu} \otimes \hat{V}_{\ulcorner, \mu} .
\end{aligned}
$$

Let us consider the action of the lower row of the Wilson loop, $\hat{E}_{b}\left(R_{1}\right)$ on the input state $\left\langle w_{1}\right|$ with the highest eigenvalue, identifying without loss of generality, as usual, the origin of the torus with the lower left corner of the loop. We get

$$
\hat{E}_{b}\left(R_{1}\right)=|\alpha|^{2\left(\mathcal{N}-R_{1}-1\right)}|\beta|^{2\left(R_{1}-1\right)} \sum_{\mu, \nu} \xi_{\llcorner, \mu} \xi_{\lrcorner, v} \operatorname{Tr}\left[\hat{H}_{\llcorner, \mu} \sigma_{x}^{R_{1}-1} \hat{H}_{\lrcorner, \nu} \Pi_{\uparrow}\right] \hat{V}_{\llcorner, \mu} \otimes \underbrace{\sigma_{x} \otimes \cdots \otimes \sigma_{x}}_{R_{1}-1} \otimes \hat{V}_{\lrcorner, \nu} \otimes \underbrace{\Pi_{\uparrow} \otimes \cdots \otimes \Pi_{\uparrow}}_{\mathcal{N}-R_{1}-1}+\ldots
$$

where the omitted terms either annihilate $\left\langle w_{1}\right|$ or are of negligible magnitude.

Some of the $\mu, v$ configurations give rise to a zero trace. Others annihilate the input vector $\left\langle w_{i}\right|$. There are only four possible valid configurations.

(1) $R_{1}$ is even, $\hat{H}_{\llcorner, \mu}=\sigma_{+}, \hat{H}_{\lrcorner, v}=\Pi_{\uparrow}$, and thus $\hat{V}_{\llcorner, \mu}=\sigma_{+}, \hat{V}_{\lrcorner, \nu}=\Pi_{\uparrow}$, and $\xi_{\llcorner, \mu} \xi_{\lrcorner, v}=|\alpha \bar{\beta}|^{2}$.

(2) $R_{1}$ is even, $\hat{H}_{\llcorner, \mu}=\Pi_{\uparrow}, \hat{H}_{\lrcorner, v}=\sigma_{-}$, and thus $\hat{V}_{\llcorner, \mu}=\Pi_{\uparrow}, \hat{V}_{\lrcorner, v}=\sigma_{+}$, and $\xi_{\llcorner, \mu} \xi_{\lrcorner, v}=|\alpha \bar{\beta}|^{2}$.

(3) $R_{1}$ is odd, $\hat{H}_{\llcorner, \mu}=\Pi_{\uparrow}, \hat{H}_{\lrcorner, v}=\Pi_{\uparrow}$, and thus $\hat{V}_{\llcorner, \mu}=\Pi_{\uparrow}, \hat{V}_{\lrcorner, v}=\Pi_{\uparrow}$, and $\xi_{\llcorner, \mu} \xi_{\lrcorner, v}=(\alpha \bar{\beta})^{2}$.

(4) $R_{1}$ is odd, $\hat{H}_{\llcorner, \mu}=\sigma_{+}, \hat{H}_{\lrcorner, \nu}=\sigma_{-}$, and thus $\hat{V}_{\llcorner, \mu}=\sigma_{+}, \hat{V}_{\lrcorner, \nu}=\sigma_{+}$, and $\xi_{\llcorner, \mu} \xi_{\lrcorner, \nu}=(\beta \bar{\alpha})^{2}$.

The leading terms of the output vector $\left\langle w_{1}\right| \hat{E}_{b}(R)$ are product vectors, with $\langle\downarrow|$ entering the loop and $\langle\uparrow|$ out of it. The two spins which are on the loop's boundaries are either flipped or not, depending on the particular configuration from the list above. We get for an even $R_{1}$

$$
\left\langle\left. w_{1}\left|\hat{E}_{b}\left(R_{1}\right)=\right| \alpha\right|^{2 \mathcal{N}}\left|\frac{\beta}{\alpha}\right|^{2 R_{1}}(\langle\downarrow| \otimes \underbrace{\langle\downarrow| \otimes \cdots \otimes\langle\downarrow|}_{R_{1}-1} \otimes\langle\uparrow| \otimes \underbrace{\langle\uparrow| \otimes \cdots \otimes\langle\uparrow|}_{\mathcal{N}-R_{1}-1}+\langle\uparrow| \otimes \underbrace{\langle\downarrow| \otimes \cdots \otimes\langle\downarrow| \otimes\langle\downarrow| \otimes\langle\uparrow| \otimes \cdots \otimes\langle\uparrow|}_{R_{1}-1})\right.
$$

and for an odd $R_{1}$

$$
\begin{aligned}
\left\langle w_{1}\right| \hat{E}_{b}\left(R_{1}\right)= & |\alpha|^{2 \mathcal{N}}\left|\frac{\beta}{\alpha}\right|^{2 R_{1}}[\left(\frac{\alpha \bar{\beta}}{|\alpha \bar{\beta}|}\right)^{2}\langle\uparrow| \otimes \underbrace{\langle\downarrow| \otimes \cdots \otimes\langle\downarrow|}_{R_{1}-1} \otimes\langle\uparrow| \otimes \underbrace{\langle\uparrow| \otimes \cdots \otimes\langle\uparrow|}_{\mathcal{N}-R_{1}-1} \\
& +\left(\frac{\beta \bar{\alpha}}{|\beta \bar{\alpha}|}\right)^{2}\langle\downarrow| \otimes \underbrace{\langle\downarrow| \otimes \cdots \otimes\langle\downarrow| \otimes\langle\downarrow| \otimes\langle\uparrow| \otimes \cdots \otimes\langle\uparrow|}_{R_{1}-1}] .
\end{aligned}
$$

We move on to the intermediate rows, with

$$
\hat{E}_{\|}\left(R_{1}\right)=|\alpha|^{2\left(\mathcal{N}-R_{1}-1\right)}|\delta|^{2\left(R_{1}-1\right)}|\beta|^{4} \sigma_{x} \otimes \underbrace{\Pi_{\downarrow} \otimes \cdots \otimes \Pi_{\downarrow}}_{R_{1}-1} \otimes \sigma_{x} \otimes \underbrace{\Pi_{\uparrow} \otimes \cdots \otimes \Pi_{\uparrow}}_{\mathcal{N}-R_{1}-1}+\ldots
$$

where, once again, the terms not included are either small enough or annihilate the input vector. The highest eigenvalue (in absolute value) is

$$
\rho_{1}^{\prime}(R)=|\alpha|^{2(\mathcal{N}-1)}\left|\frac{\beta^{2}}{\delta}\right|^{2}\left|\frac{\delta}{\alpha}\right|^{2 R_{1}}
$$

exponential in the distance $R_{1}$, just as speculated in (114), with $\Gamma=|\alpha|^{2(\mathcal{N}-1)}\left|\frac{\beta^{2}}{\delta}\right|^{2}$, and string tension $\kappa=-2 \log \left|\frac{\delta}{\alpha}\right|$, predicting an area law behavior. 
This eigenvalue is fourfold degenerate (in absolute value). Denoting by $|x= \pm 1\rangle$ the eigenvectors of $\sigma_{x}$, with eigenvalues \pm 1 , we get the four eigenvectors

$$
\left\langle w_{1}^{\prime x, x^{\prime}}(R)\right|=\langle x| \otimes \underbrace{\langle\downarrow| \otimes \cdots \otimes\langle\downarrow|}_{R_{1}-1} \otimes\left\langle x^{\prime}\right| \otimes \underbrace{\langle\uparrow| \otimes \cdots \otimes\langle\uparrow|}_{\mathcal{N}-R_{1}-1}, \quad \text { s.t. } \quad\left\langle w_{1}^{\prime x, x^{\prime}}(R)\left|\hat{E}_{\|}\left(R_{1}\right)=x x^{\prime} \rho_{1}^{\prime}(R)\right| w_{1}^{\prime x, x^{\prime}}(R)\right|
$$

Note that since the transfer matrices $\hat{E}$ and $\hat{E}_{\|}\left(R_{1}\right)$ are hermitian, $\left|v_{i}\right\rangle=\left|w_{i}\right\rangle$ and $\left|v_{i}^{\prime}(R)\right\rangle=\left|w_{i}^{\prime}(R)\right\rangle$.

Connecting with the inputs (144) and (145) and using $\langle\uparrow \mid x\rangle=1 / \sqrt{2}$ and $\langle\downarrow \mid x\rangle=x / \sqrt{2}$ we obtain for an even $R_{1}$

$$
\begin{aligned}
\left\langle w_{1}\left|\hat{E}_{b}\left(R_{1}\right)\right| v_{1}^{\prime x, x^{\prime}}\left(R_{1}\right)\right\rangle & =|\alpha|^{2 \mathcal{N}}\left|\frac{\beta}{\alpha}\right|^{2 R_{1}}\left(\langle\downarrow \mid x\rangle\left\langle\uparrow \mid x^{\prime}\right\rangle+\langle\uparrow \mid x\rangle\left\langle\downarrow \mid x^{\prime}\right\rangle\right) \\
& =\frac{1}{2}|\alpha|^{2 \mathcal{N}}\left|\frac{\beta}{\alpha}\right|^{2 R_{1}}\left(x+x^{\prime}\right) \equiv \frac{1}{2}|\alpha|^{2 \mathcal{N}}\left|\frac{\beta}{\alpha}\right|^{2 R_{1}} f_{\text {even }}\left(x, x^{\prime}\right)
\end{aligned}
$$

and for an odd one

$$
\begin{aligned}
\left\langle w_{1}\left|\hat{E}_{b}\left(R_{1}\right)\right| v_{1}^{\prime x, x^{\prime}}\left(R_{1}\right)\right\rangle & =|\alpha|^{2 \mathcal{N}}\left|\frac{\beta}{\alpha}\right|^{2 R_{1}}\left[\left(\frac{\alpha \bar{\beta}}{|\alpha \bar{\beta}|}\right)^{2}\langle\uparrow \mid x\rangle\left\langle\uparrow \mid x^{\prime}\right\rangle+\left(\frac{\beta \bar{\alpha}}{|\beta \bar{\alpha}|}\right)^{2}\langle\downarrow \mid x\rangle\left\langle\downarrow \mid x^{\prime}\right\rangle\right] \\
& =\frac{1}{2}|\alpha|^{2 \mathcal{N}}\left|\frac{\beta}{\alpha}\right|^{2 R_{1}}\left[\left(\frac{\alpha \bar{\beta}}{|\alpha \bar{\beta}|}\right)^{2}+x x^{\prime}\left(\frac{\beta \bar{\alpha}}{|\beta \bar{\alpha}|}\right)^{2}\right] \equiv \frac{1}{2}|\alpha|^{2 \mathcal{N}}\left|\frac{\beta}{\alpha}\right|^{2 R_{1}} f_{\text {odd }}\left(x, x^{\prime}\right) .
\end{aligned}
$$

We close the Wilson loop with $\hat{E}_{t}\left(R_{1}\right)$, where we consider the leading terms which do not annihilate the input vectors $\left\langle w_{1}^{\prime x, x^{\prime}}(R)\right|$ or the output vector $\left\langle w_{1}\right|$ :

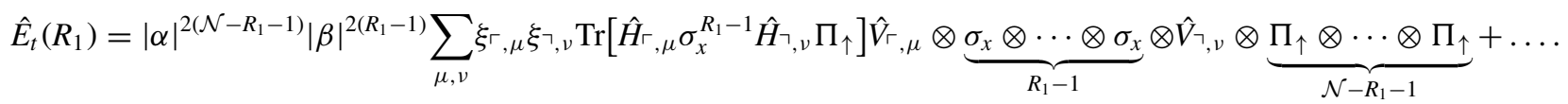

Once again there are four possible cases.

(1) $R_{1}$ is even, $\hat{H}_{\ulcorner, \mu}=\sigma_{+}, \hat{H}_{\urcorner, v}=\Pi_{\uparrow}$, and thus $\hat{V}_{\ulcorner, \mu}=\sigma_{-}, \hat{V}_{\urcorner, v}=\Pi_{\uparrow}$, and $\xi_{\ulcorner, \mu} \xi_{\urcorner, v}=|\alpha \bar{\beta}|^{2}$.

(2) $R_{1}$ is even, $\hat{H}_{\ulcorner, \mu}=\Pi_{\uparrow}, \hat{H}_{\urcorner, v}=\sigma_{-}$, and thus $\hat{V}_{\ulcorner, \mu}=\Pi_{\uparrow}, \hat{V}_{\urcorner, v}=\sigma_{-}$, and $\xi_{\ulcorner, \mu} \xi_{\urcorner, v}=|\alpha \bar{\beta}|^{2}$.

(3) $R_{1}$ is odd, $\hat{H}_{\ulcorner, \mu}=\Pi_{\uparrow}, \hat{H}_{\urcorner, v}=\Pi_{\uparrow}$, and thus $\hat{V}_{\ulcorner, \mu}=\Pi_{\uparrow}, \hat{V}_{\urcorner, v}=\Pi_{\uparrow}$, and $\xi_{\ulcorner, \mu} \xi_{\urcorner, v}=(\alpha \bar{\beta})^{2}$.

(4) $R_{1}$ is odd, $\hat{H}_{\ulcorner, \mu}=\sigma_{+}, \hat{H}_{\urcorner, v}=\sigma_{-}$, and thus $\hat{V}_{\ulcorner, \mu}=\sigma_{-}, \hat{V}_{\urcorner, v}=\sigma_{-}$, and $\xi_{\ulcorner, \mu} \xi_{\urcorner, v}=(\beta \bar{\alpha})^{2}$.

This implies that for an even $R_{1}$

$$
\hat{E}_{t}\left(R_{1}\right)\left|v_{1}\right\rangle=|\alpha|^{2 \mathcal{N}}\left|\frac{\beta}{\alpha}\right|^{2 R_{1}}(|\downarrow\rangle \otimes \underbrace{|\downarrow\rangle \otimes \cdots \otimes|\downarrow\rangle}_{R_{1}-1} \otimes|\uparrow\rangle \otimes \underbrace{|\uparrow\rangle \otimes \cdots \otimes|\uparrow\rangle}_{\mathcal{N}-R_{1}-1}+|\uparrow\rangle \otimes \underbrace{|\downarrow\rangle \otimes \cdots \otimes|\downarrow\rangle}_{R_{1}-1} \otimes|\downarrow\rangle \otimes|\uparrow\rangle \otimes \cdots \otimes|\uparrow\rangle)
$$

and for an odd $R_{1}$

$$
\begin{aligned}
\hat{E}_{t}\left(R_{1}\right)\left|v_{1}\right\rangle= & |\alpha|^{2 \mathcal{N}}\left|\frac{\beta}{\alpha}\right|^{2 R_{1}}[\left(\frac{\alpha \bar{\beta}}{|\alpha \bar{\beta}|}\right)^{2}|\uparrow\rangle \otimes \underbrace{|\downarrow\rangle \otimes \cdots \otimes|\downarrow\rangle}_{R_{1}-1} \otimes|\uparrow\rangle \otimes \underbrace{|\uparrow\rangle \otimes \cdots \otimes|\uparrow\rangle}_{\mathcal{N}-R_{1}-1} \\
& +\left(\frac{\beta \bar{\alpha}}{|\beta \bar{\alpha}|}\right)^{2}|\downarrow\rangle \otimes \underbrace{|\downarrow\rangle \otimes \cdots \otimes|\downarrow\rangle}_{R_{1}-1} \otimes|\downarrow\rangle \otimes \underbrace{|\uparrow\rangle \otimes \cdots \otimes|\uparrow\rangle}_{\mathcal{N}-R_{1}-1}],
\end{aligned}
$$

giving rise to, for an even $R_{1}$,

$$
\begin{aligned}
\left\langle w_{1}^{\prime x, x^{\prime}}\left(R_{1}\right)\left|\hat{E}_{t}\left(R_{1}\right)\right| v_{1}\right\rangle & =|\alpha|^{2 \mathcal{N}}\left|\frac{\beta}{\alpha}\right|^{2 R_{1}}\left(\langle x \mid \downarrow\rangle\left\langle x^{\prime} \mid \uparrow\right\rangle+\langle x \mid \uparrow\rangle\left\langle x^{\prime} \mid \downarrow\right\rangle\right) \\
& =\frac{1}{2}|\alpha|^{2 \mathcal{N}}\left|\frac{\beta}{\alpha}\right|^{2 R_{1}}\left(x+x^{\prime}\right)=\frac{1}{2}|\alpha|^{2 \mathcal{N}}\left|\frac{\beta}{\alpha}\right|^{2 R_{1}} f_{\text {even }}\left(x, x^{\prime}\right)
\end{aligned}
$$


and for an odd one

$$
\begin{aligned}
\left\langle w_{1}^{\prime x, x^{\prime}}\left(R_{1}\right)\left|\hat{E}_{t}\left(R_{1}\right)\right| v_{1}\right\rangle & =|\alpha|^{2 \mathcal{N}}\left|\frac{\beta}{\alpha}\right|^{2 R_{1}}\left[\left(\frac{\alpha \bar{\beta}}{|\alpha \bar{\beta}|}\right)^{2}\langle x \mid \uparrow\rangle\left\langle x^{\prime} \mid \uparrow\right\rangle+\left(\frac{\beta \bar{\alpha}}{|\beta \bar{\alpha}|}\right)^{2}\langle x \mid \downarrow\rangle\left\langle x^{\prime} \mid \downarrow\right\rangle\right] \\
& =\frac{1}{2}|\alpha|^{2 \mathcal{N}}\left|\frac{\beta}{\alpha}\right|^{2 R_{1}}\left[\left(\frac{\alpha \bar{\beta}}{|\alpha \bar{\beta}|}\right)^{2}+x x^{\prime}\left(\frac{\beta \bar{\alpha}}{|\beta \bar{\alpha}|}\right)^{2}\right]=\frac{1}{2}|\alpha|^{2 \mathcal{N}}\left|\frac{\beta}{\alpha}\right|^{2 R_{1}} f_{\text {odd }}\left(x, x^{\prime}\right) .
\end{aligned}
$$

We are finally ready to obtain the Wilson loop expectation value using the procedure of Sec. VC. We will have to slightly modify it, since in our case the highest eigenvalue of $\hat{E}_{\|}$is only degenerate in absolute value; for large loops in the thermodynamic limit we thus modify Eq. (110) to

$$
\begin{aligned}
\left\langle W\left(R_{1}, R_{2}\right)\right\rangle & =\frac{\rho_{1}^{\prime R_{2}-1}\left(R_{1}\right)}{\rho_{1}^{R_{2}+1}} \sum_{x, x^{\prime}}\left(x x^{\prime}\right)^{R_{2}-1}\left\langle w_{1}\left|\hat{E}_{b}\left(R_{1}\right)\right| v_{1}^{\prime x, x^{\prime}}\left(R_{1}\right)\right\rangle\left\langle w_{1}^{\prime x, x^{\prime}}\left(R_{1}\right)\left|\hat{E}_{t}\left(R_{1}\right)\right| v_{1}\right\rangle \\
& =\frac{1}{4}\left|\frac{\alpha \delta}{\beta^{2}}\right|^{2}\left|\frac{\delta}{\alpha}\right|^{2 R_{1} R_{2}}\left|\frac{\beta^{2}}{\alpha \delta}\right|^{2\left(R_{1}+R_{2}\right)} \sum_{x, x^{\prime}}\left(x x^{\prime}\right)^{R_{2}-1} f_{p}^{2}\left(x, x^{\prime}\right)
\end{aligned}
$$

where $p=$ even,odd is the parity of $R_{1}$.

One can already clearly see the area and perimeter dependent parts. The only thing left to do is to complete the computation of the sum, where four different cases have to be considered, corresponding to the parities of $R_{1}, R_{2}$. It is straightforward to see that if the area is even (three of the four cases) the resulting number is 8 , while if the area is odd the result is $8 \operatorname{Re}\left(\frac{\alpha \bar{\beta}}{|\alpha \bar{\beta}|}\right)^{4}$. Altogether we obtain, for large loops in the thermodynamic limit, for the $|\beta| \ll|\delta|<|\alpha|$ and $\gamma=0$ case, that

$$
\left\langle W\left(R_{1}, R_{2}\right)\right\rangle=\left\{\begin{array}{ll}
2\left|\frac{\alpha \delta}{\beta^{2}}\right|^{2}\left|\frac{\delta}{\alpha}\right|^{2 R_{1} R_{2}}\left|\frac{\beta^{2}}{\alpha \delta}\right|^{2\left(R_{1}+R_{2}\right)}, & R_{1} R_{2} \text { is even } \\
2\left|\frac{\alpha \delta}{\beta^{2}}\right|^{2}\left|\frac{\delta}{\alpha}\right|^{2 R_{1} R_{2}}\left|\frac{\beta^{2}}{\alpha \delta}\right|^{2\left(R_{1}+R_{2}\right)} \operatorname{Re}\left(\frac{\alpha \bar{\beta}}{|\alpha \bar{\beta}|}\right)^{4}, & R_{1} R_{2} \text { is odd }
\end{array} .\right.
$$

The Creutz parameter (38) is nothing but the string tension:

$$
\chi=\kappa=-2 \log \left|\frac{\delta}{\alpha}\right| \text {. }
$$

We see that we have an area law, or a confining phase, as long as $|\delta| \neq|\alpha|$. While we excluded an equality in our arguments above, indeed we will have no area law if these two parameters are equal: then, the eigenvectors of $\hat{E}$ between which the fluxes transfer will have the same eigenvalue which does not allow for an area law, in full accordance with our general discussion. If we switch $\gamma$ on, it will have two effects: one will contaminate the eigenvectors of the transfer matrix $\hat{E}$, taking them farther from product vectors until the area law is broken, as well as introduce terms in the flux-carrying transfer matrices that do not change the eigenvalue sector of $\hat{\tau}_{0}$-violating another area law criterion.

\section{Numerical examples}

We will now present a few more examples which are computed numerically, using exact contraction, on a torus with size $\mathcal{N}_{1}=8 \times \mathcal{N}_{2}=100$. We considered different choices of parameters to demonstrate different behaviors; for each, we computed the expectation value of the Wilson loop for several large loops. We extracted the parameters $\kappa_{A}$ and $\kappa_{P}$ as follows: using the expression (39) for a Wilson loop, we may define a function of $R_{2}$ depending on $R_{1}$ as a parameter:

$$
f\left(R_{2}\right)=-\log \left\langle W\left(R_{1}, R_{2}\right)\right\rangle=f_{1}\left(R_{1}\right) R_{2}+f_{0}\left(R_{1}\right) .
$$

It is a linear function, which intersects with the vertical axis at

$$
f_{0}\left(R_{1}\right)=\kappa_{P} R_{1}-\log W_{0},
$$

the slope of which is

$$
f_{1}\left(R_{1}\right)=\kappa_{A} R_{1}+\kappa_{P} .
$$

In the case of a perimeter law, the slope function will be constant, $f_{1}\left(R_{1}\right)=\kappa_{P}$, and when plotting $f\left(R_{2}\right)$ for different $R_{1}$ values parallel lines will be obtained. In the case of an area law, the lines will have different slopes. Thus, $\kappa_{A}$ and $\kappa_{P}$ may be extracted by performing linear fits to the functions $f_{1,2}\left(R_{1}\right)$. Moreover, we have extracted the Creutz parameter too.

The first set of parameters we examine is $\alpha=1, \beta=$ $0.1, \gamma=0, \delta=0.95$. This choice is within the perturbative class studied above. It shows an area law, as can be seen from Fig. 10 and the Creutz parameter $\chi=\kappa=-2 \log \left|\frac{\delta}{\alpha}\right| \approx$ 0.1025 (as shown in Fig. 11). The expected exponential dependence of the eigenvalues of $\hat{E}_{\|}(R)$ is demonstrated in Fig. 12.

Next, let us consider another example which lies within the perturbative regime: $\alpha=1, \beta=0.1, \gamma=0, \delta=1$. Here still $\gamma=0$ and $\beta$ is very small, so the eigenvectors of $\hat{E}$ would be product vectors, hence satisfying the first criterion for an area law. However, the eigenvalues of $\hat{\tau}_{0}$ are degenerate, implying no area law (the second criterion is violated). The perimeter law is clearly shown in Fig. 13, and, as one can see in Fig. 14, the eigenvalues of $\hat{E}_{\|}(R)$ have no dependence on $R$.

Finally, we consider a completely different case, where $\alpha=0.1, \beta=0.1, \gamma=1, \delta=0.3$. For this choice of parameters, the previous perturbative treatment is not valid. The eigenvalues associated with $\hat{\tau}_{0}$ are

$$
\lambda_{1} \approx 1.05, \quad \lambda_{2} \approx-0.95, \quad \lambda_{3}=0.02, \quad \lambda_{4}=0
$$



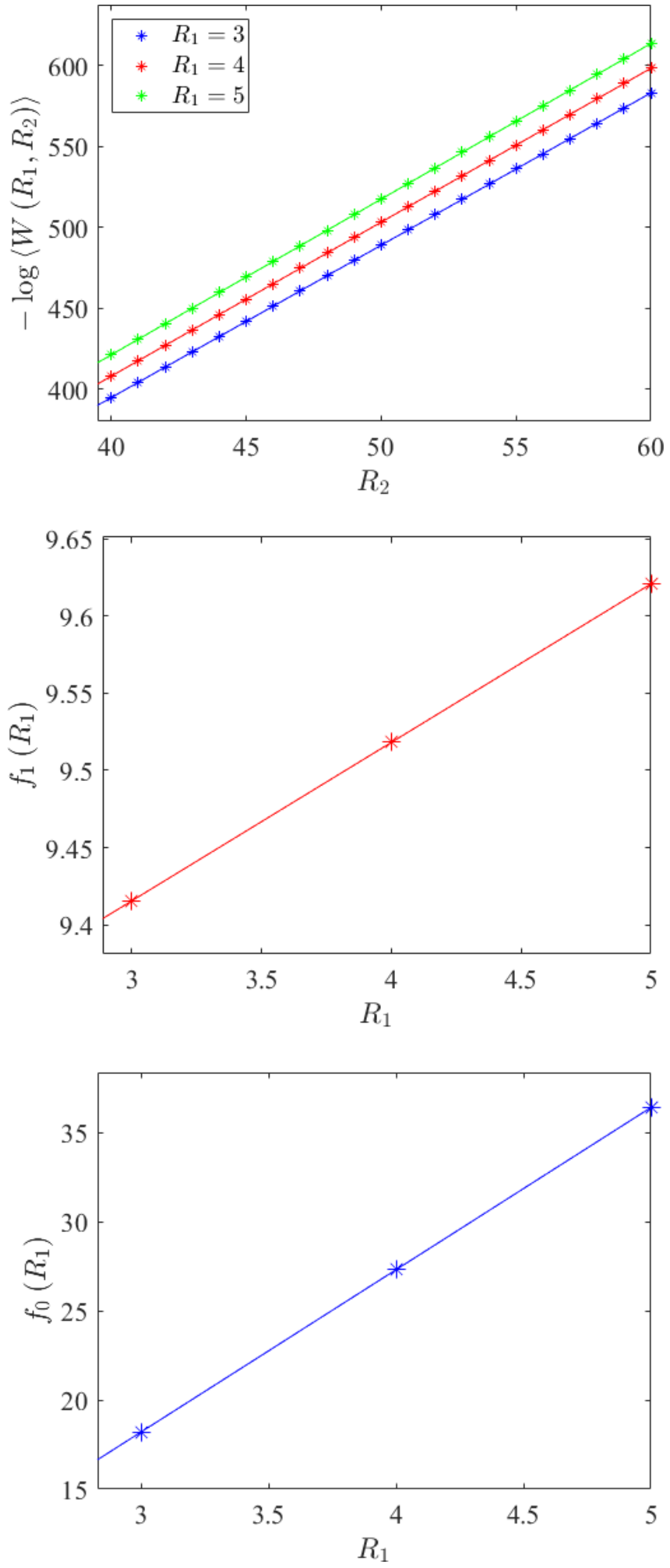

FIG. 10. $\alpha=1, \beta=0.1, \gamma=0, \delta=0.95$, which lies within the perturbative class discussed above, clearly shows an area law. It can be seen qualitatively on the top, where $-\log \left\langle W\left(R_{1}, R_{2}\right)\right\rangle$ is plotted as a function of $R_{2}$ for three different values of $R_{1}$-resulting in three nonparallel lines. And if it is hard to detect the different slopes on the top, the middle figure shows it more quantitatively: the slope function $f_{1}\left(R_{1}\right) \approx 0.126 R_{1}+9.1078$ has a nonzero slope $\kappa_{A} \approx 9.1078$, and its intersection with the vertical axis is $\kappa_{P} \approx 9.1078$, the slope of the function plotted on the bottom, $f_{0}\left(R_{1}\right)$.

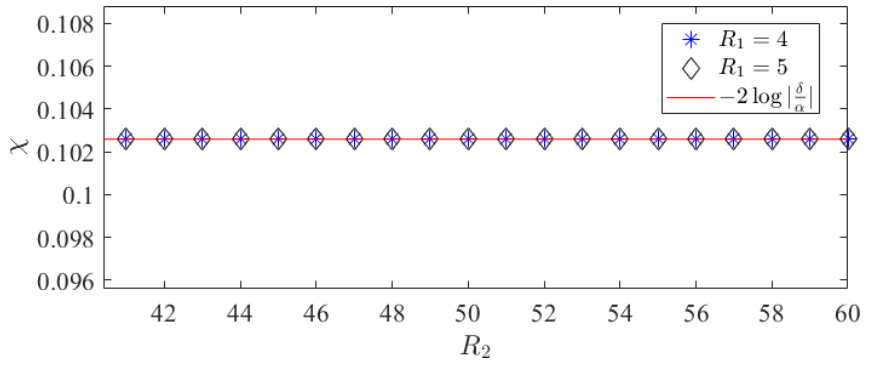

FIG. 11. Computation of the Creutz parameter $\chi\left(R_{1}, R_{2}\right)$ for $\alpha=$ $1, \beta=0.1, \gamma=0, \delta=0.95$, for different values of $R_{1}$ and $R_{2}$. As can be seen, the values converge to the predicted value (thanks to the validity of the perturbative treatment in this parameter regime) of $-2 \log \left|\frac{\delta}{\alpha}\right| \approx 0.1025$.

associated with the operators

$$
\begin{aligned}
& \hat{M}_{1} \approx\left(\begin{array}{cc}
0.6928 & 0 \\
0 & 0.7211
\end{array}\right), \\
& \hat{M}_{2} \approx\left(\begin{array}{cc}
-0.7211 & 0 \\
0 & 0.6928
\end{array}\right), \\
& \hat{M}_{3}=\frac{1}{\sqrt{2}} \sigma_{x}, \quad \hat{M}_{4}=-\frac{i}{\sqrt{2}} \sigma_{y} ;
\end{aligned}
$$

here, too, the most significant contributions are from the zeroth block with diagonal operators (the first two); however, they are far away from being projectors, hence we do not expect the eigenvectors of $\hat{E}$ to be anywhere close to product vectors.

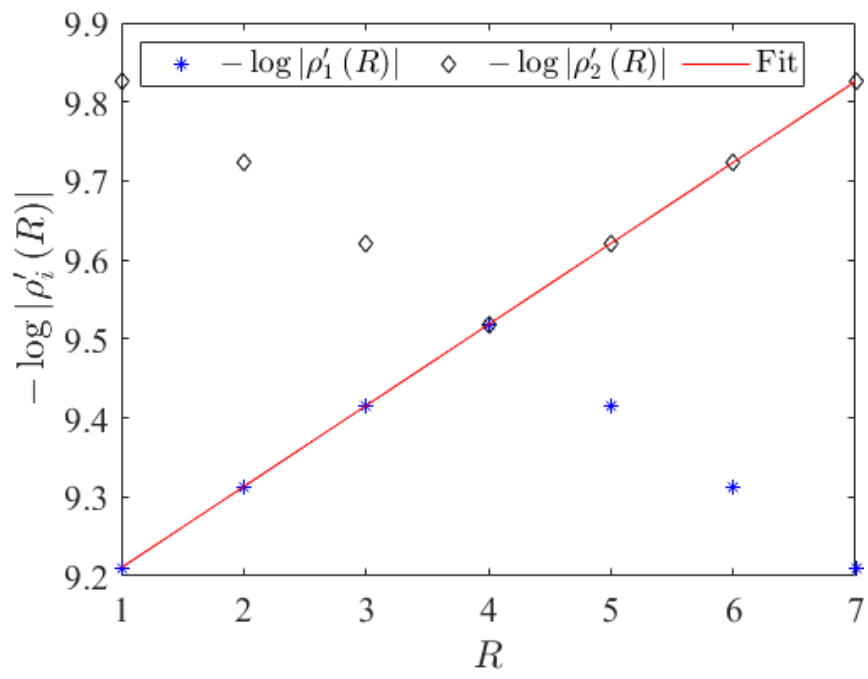

FIG. 12. In the perturbative case worked out analytically, $\alpha=$ $1, \beta=0.1, \gamma=0, \delta=0.95$, the highest eigenvalue of the intermediate transfer matrix $\hat{E}_{\|}(R)$ depends exponentially on the width $R$, as can be seen from the logarithmic plot given above, where the two highest eigenvalues (in absolute value, both degenerate in this case) are plotted for all values of $R$. The symmetric shape is due to the finiteness of the system $(\mathcal{N}=8$ in this case). For $R \leqslant 4$, the eigenvector corresponding to highest eigenvalue connects with the right input state, while for $R \geqslant 4$ the next ones are relevant-all due to the symmetry. Also shown is a linear fit, computed with respect to the parameters predicted using the perturbative treatment. 

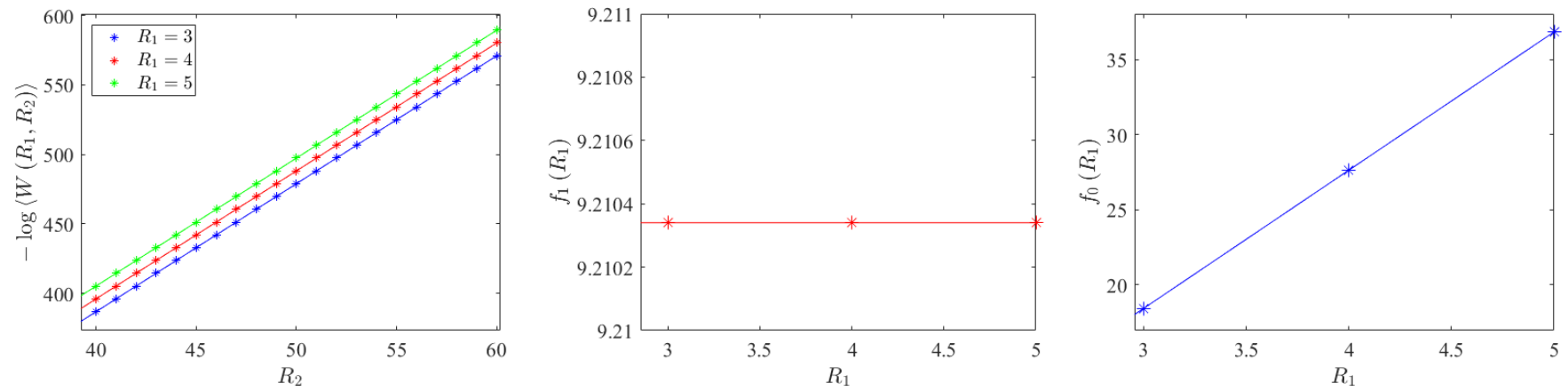

FIG. 13. The $\alpha=1, \beta=0.1, \gamma=0, \delta=1$ case does not allow for an area law because of the degeneracy in the eigenvalues of $\hat{\tau}_{0}$ corresponding to projection operators. The state shows a perimeter law, which can be seen qualitatively on the left, where $-\log \left\langle W\left(R_{1}, R_{2}\right)\right\rangle$ is plotted as a function of $R_{2}$ for three different values of $R_{1}$-resulting in three parallel lines. Quantitatively we see in the middle, where the three slopes of the three lines are plotted, that they are equal: $f_{1}\left(R_{1}\right)=\kappa_{P} \approx 9.2103$ is a constant function $\left(\kappa_{A}=0\right)$. On the right we see the fit of $f_{0}\left(R_{1}\right) \approx 9.2103\left(R_{1}-1\right)$.

Let as also consider the straight flux carrying transfer operator $\hat{\tau}_{-}$and to $\hat{\tau}_{\mid}$. We find the singular values

$$
\eta_{1} \approx 0.4472, \quad \eta_{2}=0.02, \quad \eta_{3}=\eta_{4}=0,
$$

associated, in the flux direction, with the operators

$$
\begin{aligned}
& \hat{K}_{1}=-\frac{1}{\sqrt{2}} \mathbf{1}, \quad \hat{K}_{2}=-\frac{1}{\sqrt{2}} \sigma_{x}, \\
& \hat{K}_{3}=-\frac{1}{\sqrt{2}} \sigma_{z}, \quad \hat{K}_{4}=-\frac{i}{\sqrt{2}} \sigma_{y},
\end{aligned}
$$

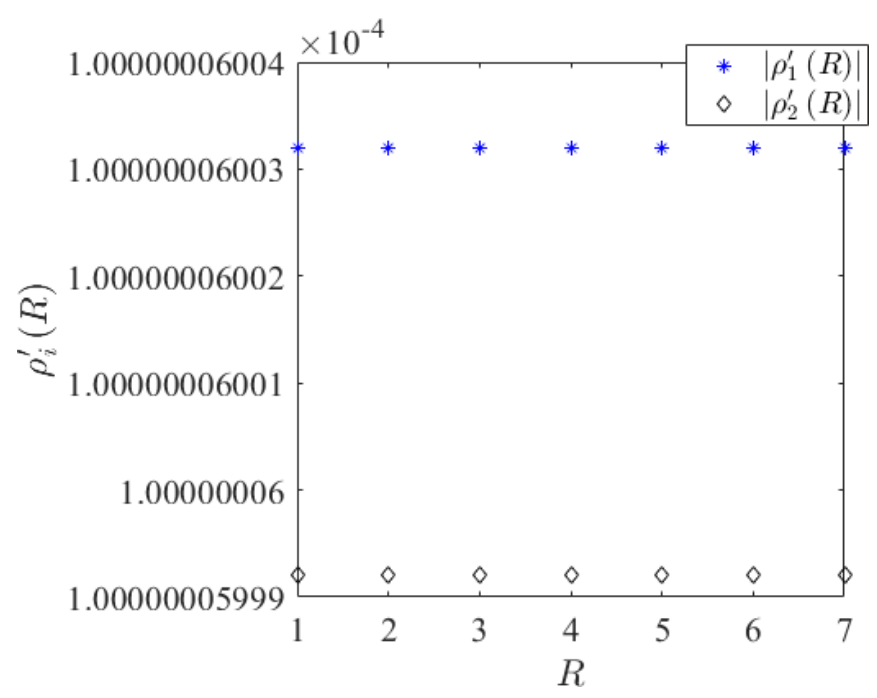

FIG. 14. The $\alpha=1, \beta=0.1, \gamma=0, \delta=1$ case does not allow for an area law because of the degeneracy in the eigenvalues of $\hat{\tau}_{0}$ corresponding to projection operators. This is also manifested by the fact that the eigenvalues of the intermediate transfer matrix, $\hat{E}_{\|}(R)$, are completely independent of the distance $R$, as illustrated here.
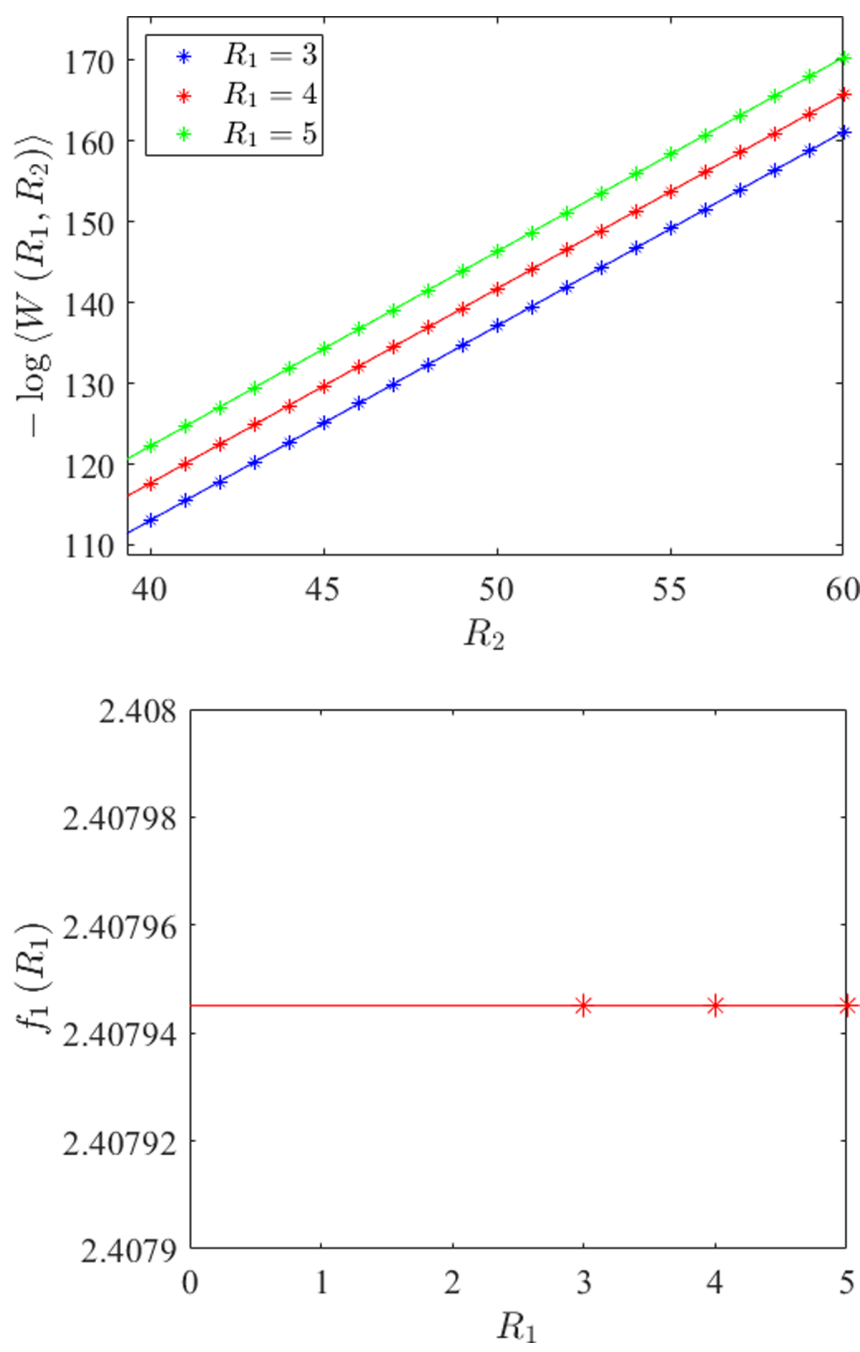

FIG. 15. The $\alpha=0.1, \beta=0.1, \gamma=1, \delta=0.3$ case shows a perimeter law, which can be seen qualitatively on the top, where $-\log \left\langle W\left(R_{1}, R_{2}\right)\right\rangle$ is plotted as a function of $R_{2}$ for three different values of $R_{1}$-resulting in three parallel lines. Quantitatively we see on the bottom, where the three slopes of the three lines are plotted, that they are equal. 


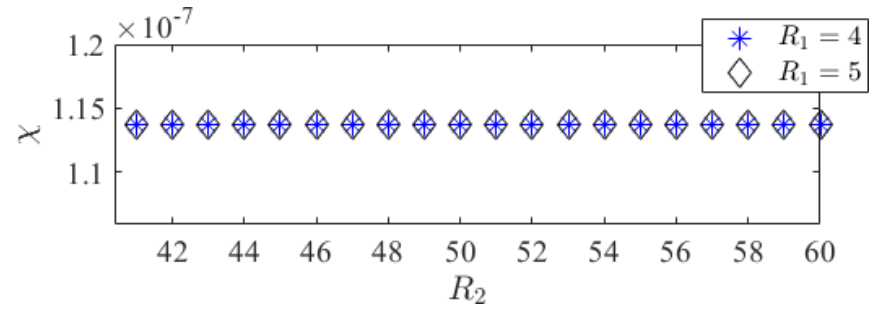

FIG. 16. Another probe for the perimeter law of the $\alpha=$ $0.1, \beta=0.1, \gamma=1, \delta=0.3$ case is the zero Creutz parameter, as plotted here (the plotted results are not exactly zero due to the fact that our loops are not very large).

and, in the direction orthogonal to the flux, with the operators

$$
\begin{aligned}
& \hat{L}_{1} \approx\left(\begin{array}{cc}
-0.3162 & 0 \\
0 & -0.9487
\end{array}\right), \quad \hat{L}_{2}=-\frac{1}{\sqrt{2}} \sigma_{x}, \\
& \hat{L}_{3} \approx\left(\begin{array}{cc}
-0.9487 & 0 \\
0 & 0.3162
\end{array}\right), \quad \hat{L}_{4}=-\frac{i}{\sqrt{2}} \sigma_{y},
\end{aligned}
$$

which imply that even if our eigenvectors were product vectors (which they are not) the most prominent contribution, coming from $\eta_{1}$, would be diagonal in the subsector (as seen from $\hat{L}_{1}$ ). Therefore all our area law criteria are violated. Indeed, this set of parameters shows a perimeter law decay of the Wilson loop, as can be seen in Fig. 15, in the zero Creutz parameter (see Fig. 16) and in the eigenvalues of $\hat{E}_{\|}(R)$ which are independent of $R$ (as shown in Fig. 17).

\section{SUMMARY}

In this paper we have seen how local properties of twodimensional lattice gauge theory PEPSs, manifested in their transfer operators (on-site) and matrices (rows), simplify their contraction and dictate their long-range Wilson loop behavior. We have related the area law with transfer matrices the eigenvectors of which are product vectors-that is, a product of local contributions of the transfer operators on each side, manifesting the lack of long-range order, as expected for a

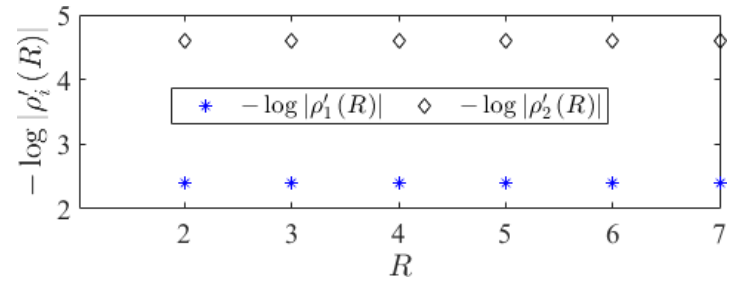

FIG. 17. In the perimeter law case of $\alpha=0.1, \beta=0.1, \gamma=$ $1, \delta=0.3$, as expected, the eigenvalues of the intermediate transfer matrix $\hat{E}_{\|}(R)$ are independent of $R$.

disordered, confining phase. The perimeter law, appearing in ordered phases, has to do with nonproduct eigenvectors, where the separate sites contribute in a correlated, longordered manner. These results may be used for detecting phases of PEPSs used for pure gauge theory studies, and for the design of PEPSs used as ansatz states for such scenarios.

One possible extension is the inclusion of dynamical matter-which is different from the current paper both in the mathematical sense (different structure of the tensors, implying different symmetry properties) and the physical one (in that case, at least with fermionic matter as in conventional standard model scenarios, the Wilson loop does not serve as an order parameter for confinement any more). This could possibly connect with the formalism of gauged Gaussian fermionic PEPSs [31,32] which can be contracted using sign-problem free Monte Carlo techniques [33,37] both for the study of further examples and application to physical models of interest.

Another important and relevant generalization is the extension to higher dimensions, where further geometry arguments have to be taken into account, potentially containing many further interesting physical and mathematical properties.

\section{ACKNOWLEDGMENTS}

I would like to thank J. I. Cirac, P. Emonts, A. Molnar, and T. B. Wahl for fruitful discussions and N. C. Hallakoun for her technical support. This research was supported by the Israel Science Foundation (Grant No. 523/20).
[1] R. Orús, A practical introduction to tensor networks: Matrix product states and projected entangled pair states, Ann. Phys. (NY) 349, 117 (2014).

[2] S. White, Density Matrix Formulation for Quantum Renormalization Groups, Phys. Rev. Lett. 69, 2863 (1992).

[3] F. Verstraete, V. Murg, and J. Cirac, Matrix product states, projected entangled pair states, and variational renormalization group methods for quantum spin systems, Adv. Phys. 57, 143 (2008).

[4] J. Cirac, D. Perez-Garcia, N. Schuch, and F. Verstraete, Matrix product states and projected entangled pair states: Concepts, symmetries, and theorems, arXiv:2011.12127 (2020).

[5] M. Sanz, M. M. Wolf, D. Pérez-García, and J. I. Cirac, Matrix product states: Symmetries and two-body Hamiltonians, Phys. Rev. A 79, 042308 (2009).
[6] A. Molnar, J. Garre-Rubio, D. Pérez-García, N. Schuch, and J. Cirac, Normal projected entangled pair states generating the same state, New J. Phys. 20, 113017 (2018).

[7] K. Wilson, Confinement of quarks, Phys. Rev. D 10, 2445 (1974).

[8] D. Gross and F. Wilczek, Ultraviolet Behavior of Non-Abelian Gauge Theories, Phys. Rev. Lett. 30, 1343 (1973).

[9] S. Aoki, Y. Aoki, D. Bečirević, T. Blum, G. Colangelo, S. Collins, M. Della Morte, P. Dimopoulos, S. Dürr, H. Fukaya, M. Golterman, S. Gottlieb, R. Gupta, S. Hashimoto, U. M. Heller, G. Herdoiza, R. Horsley, A. Jüttner, T. Kaneko, C.-J. D. Lin, et al., Flag review 2019, Eur. Phys. J. C 80, 113 (2020).

[10] K. Fukushima and T. Hatsuda, The phase diagram of dense QCD, Rep. Prog. Phys. 74, 014001 (2011). 
[11] P. Silvi, E. Rico, T. Calarco, and S. Montangero, Lattice gauge tensor networks, New J. Phys. 16, 103015 (2014).

[12] I. Kull, A. Molnar, E. Zohar, and J. Cirac, Classification of matrix product states with a local (gauge) symmetry, Ann. Phys. (NY) 386, 199 (2017).

[13] M. Bañuls, K. Cichy, J. I. Cirac, and K. Jansen, The mass spectrum of the Schwinger model with matrix product states, J. High Energy Phys. 11 (2013) 158.

[14] B. Buyens, J. Haegeman, K. Van Acoleyen, H. Verschelde, and F. Verstraete, Matrix Product States for Gauge Field Theories, Phys. Rev. Lett. 113, 091601 (2014).

[15] E. Rico, T. Pichler, M. Dalmonte, P. Zoller, and S. Montangero, Tensor Networks for Lattice Gauge Theories and Atomic Quantum Simulation, Phys. Rev. Lett. 112, 201601 (2014).

[16] H. Saito, M. C. Bañuls, K. Cichy, J. I. Cirac, and K. Jansen, The temperature dependence of the chiral condensate in the Schwinger model with Matrix Product States, arXiv:1412.0596 (2014).

[17] M. C. Bañuls, K. Cichy, J. I. Cirac, K. Jansen, and H. Saito, Thermal evolution of the Schwinger model with matrix product operators, Phys. Rev. D 92, 034519 (2015).

[18] M. C. Bañuls, K. Cichy, K. Jansen, and H. Saito, Chiral condensate in the Schwinger model with matrix product operators, Phys. Rev. D 93, 094512 (2016).

[19] B. Buyens, F. Verstraete, and K. Van Acoleyen, Hamiltonian simulation of the Schwinger model at finite temperature, Phys. Rev. D 94, 085018 (2016).

[20] M. C. Bañuls, K. Cichy, J. I. Cirac, K. Jansen, and S. Kühn, Efficient Basis Formulation for $(1+1)$-Dimensional SU(2) Lattice Gauge Theory: Spectral Calculations with Matrix Product States, Phys. Rev. X 7, 041046 (2017).

[21] S. Kühn, E. Zohar, J. I. Cirac, and M. C. Bañuls, Non-Abelian string breaking phenomena with matrix product states, J. High Energy Phys. 07 (2015) 130.

[22] T. Pichler, M. Dalmonte, E. Rico, P. Zoller, and S. Montangero, Real-Time Dynamics in U(1) Lattice Gauge Theories with Tensor Networks, Phys. Rev. X 6, 011023 (2016).

[23] B. Buyens, J. Haegeman, F. Hebenstreit, F. Verstraete, and K. Van Acoleyen, Real-time simulation of the Schwinger effect with matrix product states, Phys. Rev. D 96, 114501 (2017).

[24] M. Bañuls, K. Cichy, J. Cirac, K. Jansen, S. Kühn, and H. Saito, The multi-flavor Schwinger model with chemical potential: Overcoming the sign problem with Matrix Product States, arXiv:1611.01458 (2016).

[25] P. Silvi, E. Rico, M. Dalmonte, F. Tschirsich, and S. Montangero, Finite-density phase diagram of a $(1+1)$-D nonAbelian lattice gauge theory with tensor networks, Quantum 1, 9 (2017).

[26] M. C. Bañuls, K. Cichy, J. I. Cirac, K. Jansen, and S. Kühn, Density Induced Phase Transitions in the Schwinger Model: A Study with Matrix Product States, Phys. Rev. Lett. 118, 071601 (2017).

[27] M. C. Bañuls, K. Cichy, J. Cirac, K. Jansen, S. Kühn, and H. Saito, Towards overcoming the Monte Carlo sign problem with tensor networks, EPJ Web Conf. 137, 04001 (2017).

[28] M. C. Bañuls and K. Cichy, Review on novel methods for lattice gauge theories, Rep. Prog. Phys. 83, 024401 (2020).

[29] L. Tagliacozzo and G. Vidal, Entanglement renormalization and gauge symmetry, Phys. Rev. B 83, 115127 (2011).
[30] L. Tagliacozzo, A. Celi, and M. Lewenstein, Tensor Networks for Lattice Gauge Theories with Continuous Groups, Phys. Rev. X 4, 041024 (2014).

[31] E. Zohar, M. Burrello, T. Wahl, and J. Cirac, Fermionic projected entangled pair states and local U(1) gauge theories, Ann. Phys. (NY) 363, 385 (2015).

[32] E. Zohar, T. Wahl, M. Burrello, and J. Cirac, Projected Entangled Pair States with non-Abelian gauge symmetries: An SU(2) study, Ann. Phys. (NY) 374, 84 (2016).

[33] E. Zohar and J. I. Cirac, Combining tensor networks with Monte Carlo methods for lattice gauge theories, Phys. Rev. D 97, 034510 (2018).

[34] F. Tschirsich, S. Montangero, and M. Dalmonte, Phase diagram and conformal string excitations of square ice using gauge invariant matrix product states, SciPost Phys. 6, 21468 (2019).

[35] T. Felser, P. Silvi, M. Collura, and S. Montangero, TwoDimensional Quantum-Link Lattice Quantum Electrodynamics at Finite Density, Phys. Rev. X 10, 041040 (2020).

[36] D. Robaina, M. Banuls, and J. Cirac, Simulating 2+1D Z3 Lattice Gauge Theory with an Infinite Projected Entangled-Pair State, Phys. Rev. Lett. 126, 050401 (2021).

[37] P. Emonts, M. C. Bañuls, I. Cirac, and E. Zohar, Variational monte carlo simulation with tensor networks of a pure $\mathbb{Z}_{3}$ gauge theory in $(2+1) D$, Phys. Rev. D 102, 074501 (2020).

[38] G. Magnifico, T. Felser, P. Silvi, and S. Montangero, Lattice quantum electrodynamics in (3+1)-dimensions at finite density with tensor networks, Nat. Commun. 12, 3600 (2021).

[39] J. Haegeman, K. Van Acoleyen, N. Schuch, J. I. Cirac, and F. Verstraete, Gauging Quantum States: From Global to Local Symmetries in Many-Body Systems, Phys. Rev. X 5, 011024 (2015).

[40] E. Zohar and M. Burrello, Building projected entangled pair states with a local gauge symmetry, New J. Phys. 18, 043008 (2016).

[41] Y. Meurice, R. Sakai, and J. Unmuth-Yockey, Tensor field theory with applications to quantum computing, arXiv:2010.06539 (2020).

[42] P. Emonts and E. Zohar, Gauss Law, Minimal coupling and fermionic PEPS for lattice gauge theories, SciPost Phys. Lect. Notes, 12 (2020).

[43] M. Fannes, B. Nachtergaele, and R. F. Werner, Finitely correlated states on quantum spin chains, Commun. Math. Phys. 144, 443 (1992).

[44] S. Yang, T. B. Wahl, H.-H. Tu, N. Schuch, and J. I. Cirac, Chiral Projected Entangled-Pair State with Topological Order, Phys. Rev. Lett. 114, 106803 (2015).

[45] E. Fradkin and L. Susskind, Order and disorder in gauge systems and magnets, Phys. Rev. D 17, 2637 (1978).

[46] A. Schotte, J. Carrasco, B. Vanhecke, L. Vanderstraeten, J. Haegeman, F. Verstraete, and J. Vidal, Tensor-network approach to phase transitions in string-net models, Phys. Rev. B 100, 245125 (2019).

[47] A. Ritz-Zwilling, J.-N. Fuchs, and J. Vidal, Wegner-Wilson loops in string-nets, Phys. Rev. B 103, 075128 (2021).

[48] J. Kogut and L. Susskind, Hamiltonian formulation of Wilson's lattice gauge theories, Phys. Rev. D 11, 395 (1975).

[49] E. Zohar and M. Burrello, Formulation of lattice gauge theories for quantum simulations, Phys. Rev. D 91, 054506 (2015). 
[50] E. Zohar, J. Cirac, and B. Reznik, Quantum simulations of lattice gauge theories using ultracold atoms in optical lattices, Rep. Prog. Phys. 79, 014401 (2016).

[51] R. Brower, S. Chandrasekharan, and U.-J. Wiese, QCD as a quantum link model, Phys. Rev. D 60, 094502 (1999).

[52] V. Kasper, G. Juzeliūnas, M. Lewenstein, F. Jendrzejewski, and E. Zohar, From the Jaynes-Cummings model to non-Abelian gauge theories: A guided tour for the quantum engineer, New J. Phys. 22, 103027 (2020).

[53] L. Landau and E. Lifshitz, Quantum Mechanics: NonRelativistic Theory, Course of Theoretical Physics (Elsevier, Amsterdam, 1981).

[54] S. Weinberg, Lectures on Quantum Mechanics, 2nd ed. (Cambridge University, Cambridge, England, 2015).
[55] M. E. Rose, Elementary Theory of Angular Momentum, Dover Books on Physics and Chemistry (Dover, New York, 1995).

[56] E. Zohar, Quantum simulation of lattice gauge theories in more than one space dimension: Requirements, challenges, methods, arXiv:2106.04609 (2021).

[57] J. Kogut, An introduction to lattice gauge theory and spin systems, Rev. Mod. Phys. 51, 659 (1979).

[58] A. M. Polyakov, Gauge Fields and Strings, Contemporary Concepts in Physics (Taylor \& Francis, London, 1987).

[59] M. Creutz, Asymptotic-Freedom Scales, Phys. Rev. Lett. 45, 313 (1980).

[60] F. Verstraete, M. M. Wolf, D. Perez-Garcia, and J. I. Cirac, Criticality, the Area Law, and the Computational Power of Projected Entangled Pair States, Phys. Rev. Lett. 96, 220601 (2006). 